\title{
A NEW MODEL FOR QUANTUM DOT LIGHT EMITTING-ABSORBING DEVICES: PROOFS AND SUPPLEMENTS
}

\author{
${ }^{1}$ H. Neidhardt, ${ }^{2}$ L. Wilhelm, ${ }^{3}$ V. A. Zagrebnov \\ ${ }^{1,2}$ WIAS Berlin, Mohrenstr. 39, 10117 Berlin, Germany \\ ${ }^{3}$ Institut de Mathématiques de Marseille - UMR 7373 \\ CMI - Technopôle Château-Gombert \\ 39, rue F. Joliot Curie \\ 13453 Marseille Cedex 13, France \\ ${ }^{1}$ hagen.neidhardt@wias-berlin.de \\ ${ }^{3}$ Valentin.Zagrebnov@univ-amu.fr
}

PACS 85.60.Jb, 88.40.H, 85.35.Be, 73.63.Kv, 42.50.Pq

DOI 10.17586/2220-8054-2015-6-1-6-45

\begin{abstract}
Motivated by the Jaynes-Cummings (JC) model, we consider here a quantum dot coupled simultaneously to a reservoir of photons and to two electric leads (free-fermion reservoirs). This new Jaynes-Cummings-Leads (JCL) model permits a fermion current through the dot to create a photon flux, which describes a light-emitting device. The same model is also used to describe the transformation of a photon flux into a current of fermions, i.e. a quantum dot light-absorbing device. The key tool to obtain these results is the abstract Landauer-Büttiker formula.
\end{abstract}

Keywords: Landauer-Büttiker formula, Jaynes-Cummings model, coupling to leads, light emission, solar cells.

Received: 22 December 2014

\section{Introduction}

In the following the fermion current going through a quantum dot is analyzed as a function of

(1) the electro-chemical potentials on leads and of

(2) the contact with the external photon reservoir.

Although the latter is the canonical $J C$-interaction, the coupling of the JC model with leads needs certain precautions, if we wish to remain in the one-particle quantum mechanical Hamiltonian approach and scattering theory framework. To this end, we proposed a new Jaynes-CummingsLeads ( $J C L-)$ model [19]. This model makes possible a photon flux into the resonator, created by a fermion current through the dot; i.e. it describes a light-emitting device, as well as to transform the external photon flux into a current of fermions, which corresponds to a quantum dot lightabsorbing device.

The paper is an extended version of [19], which means that the $J C L$-model, as well as all theorems, corollaries of this article one can already find in [19], however, without any proofs. In the following article, we are going to close this gap and give full proofs of all statements. In doing so, we have added some statements absent in [19].

The paper is organized as follows. The $J C L$-model is introduced and discussed in Sections 2.1-2.7. For simplicity, we choose for the lead Hamiltonians the one-particle discrete Schrödinger operators, with constant one-site (electric) potentials on each of leads. In Section 2.5, we show that the our model fits into the framework of trace-class scattering. In Section 2.7, we verify 
the important property that the coupled Hamiltonian has no singular continuous spectrum. Our main tool for analysis of different currents is an abstract Landauer-Büttiker-type formula applied in Sections 3.1 and 3.2 to the case of the $J C L$-model. This allows us to calculate the outgoing flux of photons induced by electric current via leads. This corresponds to a light-emitting device. We also found that pumping the JCL quantum dot with a photon flux from a resonator may induce a current of fermions into the leads. This reversing imitates a quantum light-absorbing cell device. These are the main properties of our model and the main application of the Landauer-Büttikertype formula of Sections 3.1 and 3.2. They are presented in Sections 4 and 5, where we distinguish contact-induced and photon-induced fermion currents.

To describe the results of Sections 4 and 5, note that in our setup, the sample Hamiltonian is a two-level quantum dot decoupled from the one-mode photon resonator. Then, the unperturbed Hamiltonian $H_{0}$ describes a collection of four totally decoupled sub-systems: a sample, a resonator and two leads. The perturbed Hamiltonian $H$ is a fully coupled system, and the feature of our model is that it is totally (i.e. including the leads) embedded in the external electromagnetic field of the resonator. Hence, each electron can be interpreted as a fermion with internal harmonic degrees of freedom, or a Fermi-particle caring its individual photon cloud.

Similar to the "Black Box" system-leads $(S L-)$ model $\left\{H_{S L}, H_{0}\right\}$, [1], [4], it turns out that the $J C L$-model also fits into the framework of the abstract Landauer-Büttiker formula, and in particular, is a trace-class scattering system $\left\{H_{J C L}=H, H_{S L}\right\}$. The current in the $S L$-model is called the contact-induced current $J_{e l}^{c}$. This current was the subject of numerous papers, see e.g. [1,5], or [4]. Note that the current $J_{e l}$ is due to the difference of the electro-chemical potentials between two leads, but it may be zero even if this difference is not null $[12,13]$.

The fermion current in the $J C L$-model, takes into account the effect of the electron-photon interaction under the assumption that the leads are already coupled. This is called the photoninduced component $J_{e l}^{p h}$ of the total current. To the best of our knowledge, the present paper is the first which rigorously studies this phenomenon. We show that the total free-fermion current $J_{e l}$ in the $J C L$-model decomposes into a sum of the contact- and the photon-induced currents: $J_{e l}:=J_{e l}^{c}+J_{e l}^{p h}$. An extreme case is when the contact-induced current is zero, but the photoninduced component is not, c.f. Section 5.1. In this case, the flux of photons $J_{p h}$ out of the quantum dot (sample) is also non-zero, i.e. the dot serves as a light emitting device, c.f. Section 5.2. In general the $J_{p h} \neq 0$ only when the photon-induced component $J_{e l}^{p h} \neq 0$.

By choosing the parameters of the model in an suitable manner, one can get either a photon emitting or a photon absorbing system. Hence, the $J C L$-model can be used either as a light emission device or as a solar cell. Proofs of explicit formulas for fermion and photon currents $J_{l, e l}^{p h}$, $J_{p h}$ are contained in Sections 4 and 5.

Note that the $J C L$-model is called mirror symmetric if (roughly speaking) one can interchange left and right leads and the $J C L$-model remains unchanged. In Section 5 we discuss a surprising example of a mirror symmetric $J C L$-model such that the free-fermion current is zero but the model is photon emitting. This peculiarity is due to a specific choice of the photon-electron interaction, which produces fermions with internal harmonic degrees of freedom.

\section{Jaynes-Cummings quantum dot coupled to leads}

\subsection{Jaynes-Cummings model}

The starting point for the construction of our $J C L$-model is the quantum optics Jaynes-Cummings Hamiltonian $H^{J C}$. Its simplest version is a two-level system (quantum dot) with the energy spacing $\varepsilon$, defined by Hamiltonian $h_{S}$ on the Hilbert space $\mathfrak{h}_{S}=\mathbb{C}^{2}$, see e.g. [16]. It is assumed that this system is "open" and interacts with the one-mode $\omega$ photon resonator with Hamiltonian $h^{p h}$. 
Since mathematically $h^{p h}$ coincides with a quantum harmonic oscillator, the Hilbert space of the resonator is the boson Fock space $\mathfrak{h}^{p h}=\mathfrak{F}_{+}(\mathbb{C})$ over $\mathbb{C}$ and

$$
h^{p h}=\omega b^{*} b .
$$

Here, $b^{*}$ and $b$ are verifying the Canonical Commutation Relations $(C C R)$ creation and annihilation operators with domains in $\mathfrak{F}_{+}(\mathbb{C}) \simeq \ell^{2}\left(\mathbb{N}_{0}\right)$. Operator $(2.1)$ is self-adjoint on its domain:

$$
\operatorname{dom}\left(h^{p h}\right)=\left\{\left(k_{0}, k_{1}, k_{2}, \ldots\right) \in \ell^{2}\left(\mathbb{N}_{0}\right): \sum_{n \in \mathbb{N}_{0}} n^{2}\left|k_{n}\right|^{2}<\infty\right\} .
$$

Note that the canonical basis $\left\{\phi_{n}:=\left(0,0, \ldots, k_{n}=1,0, \ldots\right)\right\}_{n \in \mathbb{N}_{0}}$ in $\ell^{2}\left(\mathbb{N}_{0}\right)$ consists of eigenvectors of operator (2.1): $h^{p h} \phi_{n}=n \omega \phi_{n}$.

To model the two-level system with the energy spacing $\varepsilon$, one fixes in $\mathbb{C}^{2}$ two ortho-normal vectors $\left\{e_{0}^{S}, e_{1}^{S}\right\}$, for example $e_{0}^{S}:=\left(\begin{array}{l}0 \\ 1\end{array}\right)$ and $e_{1}^{S}:=\left(\begin{array}{l}1 \\ 0\end{array}\right)$, which are eigenvectors of Hamiltonian $h_{S}$ with eigenvalues $\left\{\lambda_{0}^{S}=0, \lambda_{1}^{S}=\varepsilon\right\}$. To this end, we set:

$$
h_{S}:=\varepsilon\left(\begin{array}{ll}
1 & 0 \\
0 & 0
\end{array}\right) \text {, }
$$

and we introduce two ladder operators:

$$
\sigma^{+}:=\left(\begin{array}{ll}
0 & 1 \\
0 & 0
\end{array}\right) \quad \text { and } \quad \sigma^{-}:=\left(\begin{array}{ll}
0 & 0 \\
1 & 0
\end{array}\right)
$$

Then, one obtains $h_{S}=\varepsilon \sigma^{+} \sigma^{-}$as well as $e_{1}^{S}=\sigma^{+} e_{0}^{S}, e_{0}^{S}=\sigma^{-} e_{1}^{S}$ and $\sigma^{-} e_{0}^{S}=0$. So, $e_{0}^{S}$ is the ground state of Hamiltonian $h_{S}$. Note that the non-interacting Jaynes-Cummings Hamiltonian $H_{0}^{J C}$ resides in the space $\mathfrak{H}^{J C}=\mathfrak{h}_{S} \otimes \mathfrak{h}^{p h}=\mathbb{C}^{2} \otimes \mathfrak{F}_{+}(\mathbb{C})$ and it is defined as the matrix operator:

$$
H_{0}^{J C}:=h_{S} \otimes I_{\mathfrak{h}^{p h}}+I_{\mathfrak{h}_{S}} \otimes h^{p h} .
$$

Here, $I_{\mathfrak{h}^{p h}}$ denotes the identity operator in the Fock space $\mathfrak{h}^{p h}$, whereas $I_{\mathfrak{h}_{S}}$ stays for the identity matrix in the space $\mathfrak{h}_{S}$.

With operators (2.3), the interaction $V_{S b}$ between quantum dot and photons (bosons) in the resonator is defined (in the rotating-wave approximation [16]) by the operator:

$$
V_{S b}:=g_{S b}\left(\sigma^{+} \otimes b+\sigma^{-} \otimes b^{*}\right) .
$$

Operators (2.4) and (2.5) define the Jaynes-Cummings model Hamiltonian:

$$
H_{J C}:=H_{0}^{J C}+V_{S b},
$$

which is a self-adjoint operator on the common domain $\operatorname{dom}\left(H_{0}^{J C}\right) \cap \operatorname{dom}\left(V_{S b}\right)$. The standard interpretation of $H_{J C}$ is that (2.6) describes an "open" two-level system interacting with an external one-mode electromagnetic field [16].

Since the one-mode resonator is able to absorb infinitely many bosons, this interpretation sounds reasonable, but one can see that the spectrum $\sigma\left(H^{J C}\right)$ of the Jaynes-Cummings model is discrete. To this end, note that the so-called number operator $\mathfrak{N}_{J C}:=\sigma^{+} \sigma^{-} \otimes I_{\mathfrak{h}^{p h}}+I_{\mathfrak{h}_{S}} \otimes b^{*} b$ commutes with $H_{J C}$. Then, since for any $n \geqslant 0$ :

$$
\mathfrak{H}_{n>0}^{J C}:=\left\{\zeta_{0} e_{0}^{S} \otimes \phi_{n}+\zeta_{1} e_{1}^{S} \otimes \phi_{n-1}\right\}_{\zeta_{0,1} \in \mathbb{C}}, \mathfrak{H}_{n=0}^{J C}:=\left\{\zeta_{0} e_{0}^{S} \otimes \phi_{0}\right\}_{\zeta_{0} \in \mathbb{C}}
$$

are eigenspaces of operator $\mathfrak{N}_{J C}$, they reduce $H_{J C}$, i.e. $H_{J C}: \mathfrak{H}_{n}^{J C} \rightarrow \mathfrak{H}_{n}^{J C}$. Note that $\mathfrak{H}^{J C}=\bigoplus_{n \geqslant 0} \mathfrak{H}_{n}^{J C}$, where each $\mathfrak{H}_{n}^{J C}$ is invariant subspace of operator (2.6). Therefore, it has 
the representation:

$$
H_{J C}=\bigoplus_{n \in \mathbb{N}_{0}} H_{J C}^{(n)}, n>1, H_{J C}^{(0)}=0
$$

Here operators $H_{J C}^{(n)}$ are the restrictions of $H_{J C}$, which act in each $\mathfrak{H}_{n}^{J C}$ as follows:

$$
\begin{aligned}
& H_{J C}^{(n)}\left(\zeta_{0} e_{0}^{S} \otimes \phi_{n}+\zeta_{1} e_{1}^{S} \otimes \phi_{n-1}\right) \\
& \quad=\left[\zeta_{0} n \omega+\zeta_{1} g_{S b} \sqrt{n}\right] e_{0}^{S} \otimes \phi_{n}+\left[\zeta_{1}(\varepsilon+(n-1) \omega)+\zeta_{0} g_{S b} \sqrt{n}\right] e_{1}^{S} \otimes \phi_{n-1} .
\end{aligned}
$$

Hence, the spectrum $\sigma\left(H_{J C}\right)=\bigcup_{n \geqslant 0} \sigma\left(H_{J C}^{(n)}\right)$. By virtue of (2.8), the spectrum $\sigma\left(H_{J C}^{(n)}\right)$ is defined for $n \geqslant 1$ by eigenvalues $E(n)$ of two-by-two matrix $\widehat{H}_{J C}^{(n)}$ acting on the coefficient space $\left\{\zeta_{0}, \zeta_{1}\right\}$ :

$$
\widehat{H}_{J C}^{(n)}\left(\begin{array}{l}
\zeta_{1} \\
\zeta_{0}
\end{array}\right)=\left(\begin{array}{cc}
\varepsilon+(n-1) \omega & g_{S b} \sqrt{n} \\
g_{S b} \sqrt{n} & n \omega
\end{array}\right)\left(\begin{array}{l}
\zeta_{1} \\
\zeta_{0}
\end{array}\right)=E(n)\left(\begin{array}{l}
\zeta_{1} \\
\zeta_{0}
\end{array}\right) .
$$

Then, (2.7) and (2.9) imply that the spectrum of the Jaynes-Cummings model Hamiltonian $H_{J C}$ is pure point:

$$
\sigma\left(H_{J C}\right)=\sigma_{p . p .}\left(H_{J C}\right)=\{0\} \cup \bigcup_{n \in \mathbb{N}}\left\{n \omega+\frac{1}{2}(\varepsilon-\omega) \pm \sqrt{(\varepsilon-\omega)^{2} / 4+g_{S b}^{2} n}\right\} .
$$

This property evidently persists for any system Hamiltonian $h_{S}$ with discrete spectrum and linear interaction (2.5) with a finite mode photon resonator [16].

We resume the above observations concerning the Jaynes-Cummings model, which is our starting point, by following remarks:

(a) The standard Hamiltonian (2.6) describes instead of flux only oscillations of photons between resonator and quantum dot, i.e. the system $h_{S}$ is not "open" enough.

(b) Since one our aim is to model a light-emitting device, the system $h_{S}$ needs an external source of energy to pump it into the dot, which will be transformed by interaction (2.5) into the outgoing photon current by pumping the resonator.

(c) To reach this aim we extend the standard Jaynes-Cummings model to our $J C L$-model by attaching to the quantum dot $h_{S}$ (2.2) two leads, which are (infinite) reservoirs of free fermions. Manipulating with electro-chemical potentials of fermions in these reservoirs we can force one of them to inject fermions in the quantum dot, whereas another one to absorb the fermions out the quantum dot with the same rate. This current of fermions throughout the dot will pump the dot and induce a photon current according scenario (b).

(d) The most subtle point is to invent a leads-dot interaction $V_{l S}$, which ensures the above mechanism and which is simple enough that one would still be able to treat this $J C L$ model using our extension of the Landauer-Büttiker formalism.

\subsection{The JCL-model}

First, let us make some general remarks and formulate certain indispensable conditions when one follows the modeling (d).

(1) Note that since the Landauer-Büttiker formalism [13] is essentially a scattering theory on a contact between two subsystems, it is developed only on a "one-particle" level. This allows one to study with this formalism only ideal (non-interacting) many-body systems. We impose this condition on many-body fermion systems (electrons) in two leads. Thus, only direct interaction between different components of the system: dot-photons $V_{S b}$ and electron-dot $V_{l S}$ are allowed. 
(2) It is well-known that fermion reservoirs are technically simpler to treat than boson ones [13]. Moreover, in the framework of our model, it is also very natural since we study electric current, although produced by "non-interacting electrons". So, below we use fermions/electrons synonymously.

(3) In spite of the precautions formulated above, the first difficulty to consider in an ideal manybody system interacting with quantized electromagnetic field (photons) is induced indirect interaction. If electrons can emit and absorb photons, it is possible for one electron to emit a photon that another electron absorbs, thus creating an indirect photon-mediated electronelectron interaction. This interaction makes it impossible to develop the Landauer-Büttiker formula, which requires a non-interacting framework.

Assumption 2.1. To solve this difficulty, we forbid in our model the photon-mediated interaction. To this end, we assume that every electron (in leads and in dot) interacts with its own distinct copy of the electromagnetic field. So, considering electrons together with their photon fields as noninteracting "composed particles", allows us to apply the Landauer-Büttiker approach. Formally, it corresponds to the "one-electron" Hilbert space $\mathfrak{h}^{e l} \otimes \mathfrak{h}^{p h}$, where $\mathfrak{h}^{p h}$ is the Hilbert space of the individual photon field. The fermion description of composed-particles $\mathfrak{h}^{\text {el }} \otimes \mathfrak{h}^{\text {ph }}$ corresponds to the antisymmetric Fock space $\mathfrak{F}_{-}\left(\mathfrak{h}^{e l} \otimes \mathfrak{h}^{p h}\right)$.

The composed-particle assumption 2.1 allows us to use the Landauer-Büttiker formalism developed for ideal many-body fermion systems. Now, we come closer to the formal description of our JCL-model with two (infinite) leads and a one-mode quantum resonator.

Recall that the Hilbert space of the Jaynes-Cummings Hamiltonian with two energy levels is $\mathfrak{H}^{J C}=\mathbb{C}^{2} \otimes \mathfrak{F}_{+}(\mathbb{C})$. The boson Fock space is constructed from a one-dimensional Hilbert space, since we consider only photons of a single fixed frequency. We model the electrons in the leads as free fermions residing on discrete semi-infinite lattices. Thus:

$$
\mathfrak{h}^{e l}=\ell^{2}(\mathbb{N}) \oplus \mathbb{C}^{2} \oplus \ell^{2}(\mathbb{N})=\mathfrak{h}_{l}^{e l} \oplus \mathfrak{h}_{S} \oplus \mathfrak{h}_{r}^{e l},
$$

is the one-particle Hilbert space for the electrons and for the dot. Here, $\mathfrak{h}_{\alpha}^{e l}, \alpha \in\{l, r\}$, are the respective Hilbert spaces of the left and right lead, while $\mathfrak{h}_{S}=\mathbb{C}^{2}$ is the Hilbert space of the quantum dot. We denote by:

$$
\left\{\delta_{n}^{\alpha}\right\}_{n \in \mathbb{N}}, \quad\left\{\delta_{n}^{S}\right\}_{j=0}^{1},
$$

the canonical basis consisting of individual lattice sites of $\mathfrak{h}_{\alpha}^{e l}, \alpha \in\{l, r\}$, and of $\mathfrak{h}_{S}$, respectively. With the Hilbert space for photons, $\mathfrak{h}^{p h}=\mathfrak{F}_{+}(\mathbb{C}) \simeq \ell^{2}\left(\mathbb{N}_{0}\right)$, we define the Hilbert space of the full system, i.e. quantum dot with leads and with the photon field, as follows:

$$
\mathfrak{H}=\mathfrak{h}^{e l} \otimes \mathfrak{h}^{p h}=\left(\ell^{2}(\mathbb{N}) \oplus \mathbb{C}^{2} \oplus \ell^{2}(\mathbb{N})\right) \otimes \ell^{2}\left(\mathbb{N}_{0}\right) .
$$

Remark 2.2. Note that the structure of full space (2.11) takes into account the condition 2.1 and produces composed fermions via the last tensor product. It also manifests that electrons in the dot as well as those in the leads are composed with photons. This is different than the picture imposed by the the Jaynes-Cummings model, when only the dot is composed with photons:

$$
\mathfrak{H}=\ell^{2}(\mathbb{N}) \oplus \mathbb{C}^{2} \otimes \ell^{2}\left(\mathbb{N}_{0}\right) \oplus \ell^{2}(\mathbb{N}) \quad, \quad \mathfrak{H}^{J C}=\mathbb{C}^{2} \otimes \ell^{2}\left(\mathbb{N}_{0}\right),
$$

see (2.4), (2.5) and (2.6), where $\mathfrak{H}^{J C}=\mathfrak{h}_{S} \otimes \mathfrak{h}^{p h}$. The next step is a choice of interactions between subsystems: dot-resonator-leads.

According to (2.10), the decoupled leads-dot Hamiltonian is the matrix operator:

$$
h_{0}^{e l}=\left(\begin{array}{ccc}
h_{l}^{e l} & 0 & 0 \\
0 & h_{S} & 0 \\
0 & 0 & h_{r}^{e l}
\end{array}\right) \text { on } u=\left(\begin{array}{c}
u_{l} \\
u_{S} \\
u_{r}
\end{array}\right),\left\{u_{\alpha} \in \ell^{2}(\mathbb{N})\right\}_{\alpha \in\{l, r\}}, u_{S} \in \mathbb{C}^{2} \text {, }
$$


where $h_{\alpha}^{e l}=-\Delta^{D}+v_{\alpha}$ with a constant potential bias $v_{\alpha} \in \mathbb{R}, \alpha \in\{l, r\}$, and $h_{S}$ can be any self-adjoint two-by-two matrix with eigenvalues $\left\{\lambda_{0}^{S}, \lambda_{1}^{S}:=\lambda_{0}^{S}+\varepsilon\right\}, \varepsilon>0$, and eigenvectors $\left\{e_{0}^{S}, e_{1}^{S}\right\}$, cf (2.2). Here, $\Delta^{D}$ denotes the discrete Laplacian on $\ell^{2}(\mathbb{N})$ with homogeneous Dirichlet boundary conditions given by:

$$
\begin{aligned}
\left(\Delta^{D} f\right)(x) & :=f(x+1)-2 f(x)+f(x-1), \quad x \in \mathbb{N}, \\
\operatorname{dom}\left(\Delta^{D}\right) & :=\left\{f \in \ell^{2}\left(\mathbb{N}_{0}\right): f(0):=0\right\},
\end{aligned}
$$

which is obviously a bounded self-adjoint operator. Notice that $\sigma\left(\Delta^{D}\right)=[0,4]$.

We define the lead-dot interaction for coupling $g_{e l} \in \mathbb{R}$ by the matrix operator acting in (2.10) as follows:

$$
v_{e l}=g_{e l}\left(\begin{array}{ccc}
0 & \left\langle\cdot, \delta_{0}^{S}\right\rangle \delta_{1}^{l} & 0 \\
\left\langle\cdot, \delta_{1}^{l}\right\rangle \delta_{0}^{S} & 0 & \left\langle\cdot, \delta_{1}^{r}\right\rangle \delta_{1}^{S} \\
0 & \left\langle\cdot, \delta_{1}^{S}\right\rangle \delta_{1}^{r} & 0
\end{array}\right),
$$

where non-trivial off-diagonal entries are projection operators in the Hilbert space (2.10) with the scalar product $u, v \mapsto\langle u, v\rangle$ for $u, v \in \mathfrak{h}^{e l}$. Here, $\left\{\delta_{0}^{S}, \delta_{1}^{S}\right\}$ is ortho-normal basis in $\mathfrak{h}_{S}^{e l}$, which in general may be different from $\left\{e_{0}^{S}, e_{1}^{S}\right\}$. Hence, interaction (2.13) describes quantum tunneling between leads and the dot via contact sites of the leads, which are supports of $\delta_{1}^{l}$ and $\delta_{1}^{r}$.

Then we define the Hamiltonian for the system of interacting leads and dot as $h^{e l}:=$ $h_{0}^{e l}+v_{e l}$. Here, both $h_{0}^{e l}$ and $h^{e l}$ are bounded self-adjoint operators on $\mathfrak{h}^{\text {el }}$.

Recall that photon Hamiltonian in the one-mode resonator is defined by operator $h^{p h}=$ $\omega b^{*} b$ with domain in the Fock space $\mathfrak{F}_{+}(\mathbb{C}) \simeq \ell^{2}\left(\mathbb{N}_{0}\right),(2.1)$. We denote the canonical basis in $\ell^{2}\left(\mathbb{N}_{0}\right)$ by $\left\{\Upsilon_{n}\right\}_{n \in \mathbb{N}_{0}}$. Then for the spectrum of $h^{p h}$ one obviously gets:

$$
\sigma\left(h^{p h}\right)=\sigma_{p p}\left(h^{p h}\right)=\bigcup_{n \in \mathbb{N}_{0}}\{n \omega\} .
$$

We introduce the following decoupled Hamiltonian $H_{0}$, which describes the system when the leads are decoupled from the quantum dot and the electron does not interact with the photon field:

$$
H_{0}:=H_{0}^{e l}+H^{p h}
$$

where

$$
H_{0}^{e l}:=h_{0}^{e l} \otimes I_{\mathfrak{h}^{p h}} \quad \text { and } \quad H^{p h}:=I_{\mathfrak{h}^{e l}} \otimes h^{p h} .
$$

The operator $H_{0}$ is self-adjoint on $\operatorname{dom}\left(H_{0}\right)=\operatorname{dom}\left(I_{\mathfrak{h}} \mathfrak{h}^{e l} \otimes h^{p h}\right)$. Recall that $h_{0}^{e l}$ and $h^{p h}$ are bounded self-adjoint operators. Hence, $H_{0}^{e l}$ and $H^{e l}$ are semi-bounded from below, which yields that $H_{0}$ is semi-bounded from below.

The interaction of the photons and the electrons in the quantum dot is given by the coupling of the dipole moment of the electrons to the electromagnetic field in the rotating wave approximation. Namely:

$$
V_{p h}=g_{p h}\left(\left(\cdot, e_{0}^{S}\right) e_{1}^{S} \otimes b+\left(\cdot, e_{1}^{S}\right) e_{0}^{S} \otimes b^{*}\right),
$$

for some coupling constant $g_{p h} \in \mathbb{R}$. The total Hamiltonian is given by:

$$
H:=H^{e l}+H^{p h}+V_{p h}=H_{0}+V_{e l}+V_{p h},
$$

where $H^{e l}:=h^{e l} \otimes I_{\mathfrak{h}^{p h}}$ and $V_{e l}:=v_{e l} \otimes I_{\mathfrak{h}^{p h}}$.

In the following, we call $\mathcal{S}=\left\{H, H_{0}\right\}$ the Jaynes-Cummings-leads system, in short $J C L$ model, which we are going to analyze. In particular, we are interested in the electron and photon currents for that system. The analysis will be based on the abstract Landauer-Büttiker formula, cf. $[1,13]$.

Lemma 2.3. $H$ is bounded from below self-adjoint such that $\operatorname{dom}(H)=\operatorname{dom}\left(H_{0}\right)$. 
Proof. Let $c \geqslant 2$. Then,

$$
\left\|b \Upsilon_{n}\right\|^{2} \leqslant\left\|b^{*} \Upsilon_{n}\right\|^{2}=n+1 \leqslant c^{-1} n^{2}+c, \quad n \in \mathbb{N}_{0} .
$$

Consider elements $f \in \mathfrak{h}_{S} \otimes \mathfrak{h}^{p h} \cap \operatorname{dom}\left(I_{\mathfrak{h}}{ }^{e l} \otimes h^{p h}\right)$ with the following:

$$
f=\sum_{j, l} \beta_{j l} e_{j} \otimes \Upsilon_{l}, \quad j \in\{0,1\}, \quad l \in \mathbb{N}_{0},
$$

which are dense in $\mathfrak{H}^{J C}:=\mathfrak{h}_{S}^{e l} \otimes h^{p h}$. Then, $\|f\|^{2}=\sum_{j, l}\left|\beta_{j l}\right|^{2}$ and $\left\|\left(I_{\mathfrak{h}}^{e l} \otimes b^{*} b\right) f\right\|^{2}=\sum_{j, l=1}\left|\beta_{j l}\right|^{2} l^{2}$. We obtain the following:

$$
\begin{aligned}
& \left\|\left(\left(\cdot, e_{1}^{S}\right) e_{0}^{S} \otimes b\right) f\right\|^{2} \leqslant \sum_{j, l}\left|\beta_{j l}\right|^{2}\left\|b \Upsilon_{l}\right\|^{2} \leqslant \\
& \sum_{j, l}\left|\beta_{j l}\right|^{2}\left(c^{-1} l^{2}+c\right)=c^{-1}\left\|\left(I_{\mathfrak{h}^{e l}} \otimes b^{*} b\right) f\right\|^{2}+c\|f\|^{2}
\end{aligned}
$$

Similarly,

$$
\left\|\left(\left(\cdot, e_{1}^{S}\right) e_{0}^{S} \otimes b^{*}\right) f\right\|^{2} \leqslant c^{-1}\left\|\left(I_{\mathfrak{h}}{ }^{e l} \otimes b^{*} b\right) f\right\|^{2}+c\|f\|^{2} .
$$

If $c \geqslant 2$ is large enough, then we obtain that $V_{p h}$ is dominated by $H^{p h}$ with relative bound less than one. Hence, $H$ is self-adjoint and $\operatorname{dom}\left(H_{0}\right)=\operatorname{dom}(H)$. Since $H_{0}^{e l}$ and $V_{e l}$ are bounded and $H^{p h}$ is self-adjoint and bounded from below, it follows that $H=H_{0}^{e l}+H^{p h}+V_{e l}+V_{p h}$ is bounded from below [17, Thm. V.4.1].

\subsection{Time reversible symmetric systems}

A system described by the Hamiltonian $H$ is called time reversible symmetric if there is a conjugation $\Gamma$ defined on $\mathfrak{H}$ such that $\Gamma H=H \Gamma$. Recall that $\Gamma$ is a conjugation if the conditions $\Gamma^{2}=I$ and $(\Gamma f, \Gamma g)=\overline{(f, g)}, f, g \in \mathfrak{H}$.

Let $\mathfrak{h}_{n}^{p h}, n \in \mathbb{N}_{0}$, the subspace spanned by the eigenvector $\Upsilon_{n}$ in $\mathfrak{h}^{p h}$. We set:

$$
\mathfrak{H}_{n_{\alpha}}:=\mathfrak{h}_{\alpha}^{e l} \otimes \mathfrak{h}_{n}^{p h}, \quad n \in \mathbb{N}_{0}, \quad \alpha \in\{l, r\} .
$$

Notice that

$$
\mathfrak{H}=\bigoplus_{n \in \mathbb{N}_{0}, \alpha \in\{l, r\}} \mathfrak{H}_{n_{\alpha}}
$$

Definition 2.4. The $J C L$-model is called time reversible symmetric if there is a conjugation $\Gamma$ acting on $\mathfrak{H}$ such that $H$ and $H_{0}$ are time reversible symmetric and the subspaces $\mathfrak{H}_{n_{\alpha}}, n \in \mathbb{N}_{0}$, $\alpha \in\{l, r\}$, reduces $\Gamma$.

Example 2.5. Let $\gamma_{\alpha}^{e l}$ and $\gamma_{S}^{e l}$ be conjugations defined by the following:

$$
\gamma_{\alpha}^{e l} f_{\alpha}:=\overline{f_{\alpha}}:=\left\{\overline{f_{\alpha}(k)}\right\}_{k \in \mathbb{N}}, \quad f_{\alpha} \in \mathfrak{h}_{\alpha}^{e l}, \quad \alpha \in\{l, r\},
$$

and

We set $\gamma^{e l}:=\gamma_{l}^{e l} \oplus \gamma_{S}^{e l} \oplus \gamma_{r}^{e l}$. Further, we set:

$$
\gamma_{S}^{e l} f_{S}=\gamma_{S}^{e l}\left(\begin{array}{l}
f_{S}(0) \\
f_{S}(1)
\end{array}\right):=\left(\overline{\frac{f_{S}(0)}{f_{S}(1)}}\right)
$$

$$
\gamma^{p h} \psi:=\bar{\psi}=\{\overline{\psi(n)}\}_{n \in \mathbb{N}_{0}}, \quad \psi \in \mathfrak{h}^{p h} .
$$

Let $\Gamma:=\gamma^{e l} \otimes \gamma^{p h}$. One easily checks that $\Gamma$ is a conjugation on $\mathfrak{H}=\mathfrak{h}^{e l} \otimes \mathfrak{h}^{p h}$.

Lemma 2.6. Let $\gamma_{\alpha}^{e l}, \alpha \in\{S, l, r\}$, and $\gamma^{\text {ph }}$ be given by Example 2.5.

(i) If the conditions $\gamma_{S}^{e l} e_{0}^{S}=e_{0}^{S}$ and $\gamma_{S}^{e l} e_{1}^{S}=e_{1}^{S}$ are satisfied, then $H_{0}$ is time reversible symmetric with respect to $\Gamma$ and, moreover, the subspaces $\mathfrak{H}_{n_{\alpha}}, n \in \mathbb{N}_{0}, \alpha \in\{l, r\}$, reduces $\Gamma$. 
(ii) If in addition the conditions $\gamma_{S}^{e l} \delta_{0}^{S}=\delta_{0}^{S}$ and $\gamma_{S}^{e l} \delta_{1}^{S}=\delta_{1}^{S}$ are satisfied, then JCL-model is time reversible symmetric.

Proof. (i) Obviously we have

$$
\gamma_{\alpha}^{e l} h_{\alpha}^{e l}=h_{\alpha}^{e l} \gamma_{\alpha}^{e l}, \quad \alpha \in\{l, r\}, \quad \text { and } \quad \gamma^{p h} h^{p h}=h^{p h} \gamma^{p h}
$$

If $\gamma_{S}^{e l} e_{0}^{S}=e_{0}^{S}$ and $\gamma_{S}^{e l} e_{1}^{S}=e_{1}^{S}$ are satisfied, then $\gamma_{S}^{e l} h_{S}^{e l}=h_{S}^{e l} \gamma_{S}^{e l}$ which yields $\gamma^{e l} h_{0}^{e l}=h_{0}^{e l} \gamma^{e l}$ and, hence, $\Gamma H_{0}=\Gamma H_{0}$. Since $\gamma^{e l} \mathfrak{h}_{\alpha}^{e l}=\mathfrak{h}_{\alpha}^{e l}$ and $\gamma^{p h} \mathfrak{h}^{p h}=\mathfrak{h}^{p h}$ one gets $\Gamma \mathfrak{H}_{n_{\alpha}}=\mathfrak{H}_{n_{\alpha}}$ which shows that $\mathfrak{H}_{n_{\alpha}}$ reduces $\Gamma$.

(ii) Notice that $\gamma_{\alpha}^{e l} \delta_{1}^{\alpha}=\delta_{1}^{\alpha}, \alpha \in\{l, r\}$. If in addition the conditions $\gamma_{S}^{e l} \delta_{0}^{S}=\delta_{0}^{S}$ and $\gamma_{S}^{e l} \delta_{1}^{S}=\delta_{1}^{S}$ are satisfied, then $\gamma^{e l} v_{e l}=v_{e l} \gamma^{e l}$ is valid, which yields $\gamma^{e l} h^{e l}=h^{e l} \gamma^{e l}$. Hence, $\Gamma H=H \Gamma$. Together with (i), this proves that the $J C L$-model is time reversible symmetric.

Choosing the following:

$$
e_{0}^{S}:=\left(\begin{array}{l}
1 \\
0
\end{array}\right), \quad e_{1}^{S}:=\left(\begin{array}{l}
0 \\
1
\end{array}\right), \quad \delta_{0}^{S}:=\frac{1}{\sqrt{2}}\left(\begin{array}{l}
1 \\
1
\end{array}\right), \quad \delta_{1}^{S}:=\frac{1}{\sqrt{2}}\left(\begin{array}{c}
1 \\
-1
\end{array}\right)
$$

one satisfies the condition $\gamma_{S}^{e l} e_{0}^{S}=e_{0}^{S}$ and $\gamma_{S}^{e l} e_{1}^{S}=e_{1}^{S}$ as well as $\gamma_{S}^{e l} e_{0}^{S}=e_{0}^{S}$ and $\gamma_{S}^{e l} e_{1}^{S}=e_{1}^{S}$.

\subsection{Mirror symmetric systems}

A unitary operator $U$ acting on $\mathfrak{H}$ is called a mirror symmetry if the following conditions are met:

$$
U \mathfrak{H}_{n_{\alpha}}=\mathfrak{H}_{n_{\alpha^{\prime}}}, \quad \alpha, \alpha^{\prime} \in\{l, r\}, \quad \alpha \neq \alpha^{\prime}
$$

are satisfied. In particular, this yields $U \mathfrak{H}^{J C}=\mathfrak{H}^{J C}, \mathfrak{H}^{J C}:=\mathfrak{h}_{S}^{e l} \otimes \mathfrak{h}^{p h}$.

Definition 2.7. The $J C L$-model is called mirror symmetric if there is a mirror symmetry commuting with $H_{0}$ and $H$.

One can easily verify that if $H_{0}$ is mirror symmetric, then

$$
H_{n_{\alpha^{\prime}}} U=U H_{n_{\alpha}}, \quad n \in \mathbb{N}_{0}, \quad \alpha, \alpha^{\prime} \in\{l, r\}, \quad \alpha \neq \alpha^{\prime},
$$

where

$$
H_{n_{\alpha}}:=h_{\alpha}^{e l} \otimes I_{\mathfrak{h}_{n}^{p h}}+I_{\mathfrak{h}_{\alpha}^{e l}} \otimes h_{n}^{p h}=h_{\alpha}^{e l}+n \omega, \quad n \in \mathbb{N}_{0}, \quad \alpha, \alpha^{\prime} \in\{l, r\}, \quad \alpha \neq \alpha^{\prime} .
$$

In particular, this yields that $v_{\alpha}=v_{\alpha^{\prime}}$. Moreover, one gets $U H_{S}=H_{S} U$ where $H_{S}:=h_{S}^{e l} \otimes I_{\mathfrak{h}^{p h}}+$ $I_{\mathfrak{h}^{e l}} \otimes h^{p h}$.

Notice that if $H$ and $H_{0}$ commute with the same mirror symmetry $U$, then also the operator $H_{c}:=h^{e l} \otimes I_{\mathfrak{h}^{p h}}+I_{\mathfrak{h}}{ }^{e l} \otimes h^{p h}$ also commutes with $U$, i.e, is mirror symmetric.

Example 2.8. Let $\mathcal{S}=\left\{H, H_{0}\right\}$ be the $J C L$-model. Let $v_{l}=v_{r}$ and let $e_{0}^{S}$ and $e_{1}^{S}$ as well as $\delta_{0}^{S}$ and $\delta_{1}^{S}$ be given by (2.19). We set:

$$
u_{S}^{e l} e_{0}^{S}:=e_{0}^{S} \quad \text { and } \quad u_{S}^{e l} e_{1}^{S}=-e_{1}^{S},
$$

as well as

$$
u^{p h} \Upsilon_{n}=e^{-i n \pi} \Upsilon_{n}, \quad n \in \mathbb{N}_{0} .
$$

Obviously, $U_{S}:=u_{S}^{e l} \otimes u^{p h}$ defines a unitary operator on $\mathfrak{H}^{J C}$. Straightforward computation shows that:

$$
U_{S} H_{S}=H_{S} U_{S} \quad \text { and } \quad U_{S} V_{p h}=V_{p h} U_{S} .
$$

Furthermore, we set:

$$
u_{r l}^{e l} l_{n}^{l}:=\delta_{n}^{r}, \quad \text { and } \quad u_{l r}^{e l} \delta_{n}^{r}=\delta_{n}^{l}, \quad n \in \mathbb{N},
$$


and

$$
u^{e l}:=\left(\begin{array}{ccc}
0 & 0 & u_{l r}^{e l} \\
0 & u_{S}^{e l} & 0 \\
u_{l r}^{e l} & 0 & 0
\end{array}\right)
$$

We thus have the following:

$$
v_{e l} u^{e l}\left(\begin{array}{c}
f_{l} \\
f_{S} \\
f_{r}
\end{array}\right)=\left(\begin{array}{c}
<f_{S},\left(u_{S}^{e l}\right)^{*} \delta_{0}^{S}>\delta_{1}^{l} \\
<f_{r},\left(u_{l r}^{e l}\right)^{*} \delta_{1}^{l}>\delta_{0}^{S}+<f_{l},\left(u_{r l}^{e l}\right)^{*} \delta_{1}^{r}>\delta_{1}^{S} \\
<f_{S},\left(u_{S}^{e l}\right)^{*} \delta_{1}^{S}>\delta_{1}^{r}
\end{array}\right)
$$

Since $\delta_{0}^{S}:=\frac{1}{\sqrt{2}}\left(e_{0}^{S}+e_{1}^{S}\right)$ and $\delta_{1}^{S}:=\frac{1}{\sqrt{2}}\left(e_{0}^{S}-e_{1}^{S}\right)$ we get from (2.20)

$$
\left(u_{S}^{e l}\right)^{*} \delta_{0}^{S}=\delta_{1}^{S} \quad \text { and } \quad\left(u_{S}^{e l}\right)^{*} \delta_{1}^{S}=\delta_{0}^{S} .
$$

Obviously, we then have

$$
\left(u_{l r}^{e l}\right)^{*} \delta_{1}^{l}=\delta_{1}^{r} \quad\left(u_{r l}^{e l}\right)^{*} \delta_{1}^{r}=\delta_{1}^{l} .
$$

Inserting (2.25) and (2.26) into (2.24), we find

$$
v_{e l} u^{e l}\left(\begin{array}{c}
f_{l} \\
f_{S} \\
f_{r}
\end{array}\right)=\left(\begin{array}{c}
<f_{S}, \delta_{1}^{S}>\delta_{1}^{l} \\
<f_{r}, \delta_{1}^{r}>\delta_{0}^{S}+<f_{l}, \delta_{1}^{l}>\delta_{1}^{S} \\
<f_{S}, \delta_{0}^{S}>\delta_{1}^{r}
\end{array}\right)
$$

Further, we have:

$$
u^{e l} v_{e l}\left(\begin{array}{c}
f_{l} \\
f_{S} \\
f_{r}
\end{array}\right)=\left(\begin{array}{c}
<f_{S}, \delta_{1}^{S}>\delta_{1}^{l} \\
<f_{l}, \delta_{1}^{l}>\delta_{1}^{S}+<f_{r}, \delta_{1}^{r}>\delta_{0}^{S} \\
<f_{s}, \delta_{0}^{S}>\delta_{1}^{r}
\end{array}\right) .
$$

Comparing (2.27) and (2.28), we get $u^{e l} v_{e l}=v_{e l} u^{e l}$. Setting $U:=u^{e l} \otimes u^{p h}$ one immediately proves that $U H_{0}=H_{0} U$ and $U H=H U$. Since $U \mathfrak{H}_{n_{\alpha}}=\mathfrak{H}_{n_{\alpha^{\prime}}}$, it is satisfied that $\mathcal{S}$ is mirror symmetric.

We note that Example $2.8 \mathcal{S}$ is also time-reversible symmetric.

\subsection{Spectral properties of $H$ : first part}

In the following, our goal is to apply the Landauer-Büttiker formula to the $J C L$-model. By $\mathfrak{L}_{p}(\mathfrak{H})$, $1 \leqslant p \leqslant \infty$, we denote in the following the Schatten-v.Neumann ideals.

Proposition 2.9. If $\mathcal{S}=\left\{H, H_{0}\right\}$ is the JCL-model, then $(H+i)^{-1}-\left(H_{0}+i\right)^{-1} \in \mathfrak{L}_{1}(\mathfrak{H})$. In particular, the absolutely continuous parts $H^{a c}$ and $H_{0}^{a c}$ are unitarily equivalent.

Proof. We have

$$
\begin{aligned}
& (H+i)^{-1}-\left(H_{0}+i\right)^{-1}=\left(H_{0}+i\right)^{-1} V(H+i)^{-1}= \\
& \quad\left(H_{0}+i\right)^{-1} V\left(H_{0}+i\right)^{-1}-\left(H_{0}+i\right)^{-1} V\left(H_{0}+i\right)^{-1} V(H+i)^{-1},
\end{aligned}
$$

where $V=H-H_{0}=V_{e l}+V_{p h}$. Taking into account Lemma 2.3, it suffices to prove that $\left(H_{0}+i\right)^{-1} V\left(H_{0}+i\right)^{-1} \in \mathfrak{L}_{1}(\mathfrak{H})$. Using the spectral decomposition of $h^{p h}$ with respect to $\mathfrak{h}^{p h}=$ $\bigoplus_{n \in \mathbb{N}_{0}} \mathfrak{h}_{n}^{p h}$, where $\mathfrak{h}_{n}^{p h}$ are the subspaces spanned by $\Upsilon_{n}$, we obtain the following:

$$
\left(H_{0}+i\right)^{-1}=\bigoplus_{n \in \mathbb{N}_{0}}\left(h_{0}^{e l}+n \omega+i\right)^{-1} \otimes I_{\mathfrak{h}_{n}^{p h}}
$$


We have $\left(H_{0}+i\right)^{-1} V\left(H_{0}+i\right)^{-1}=\left(H_{0}+i\right)^{-1}\left(V_{e l}+V_{p h}\right)\left(H_{0}+i\right)^{-1}$. Since $v_{e l}$ is a finite rank operator, we have $\left\|v_{e l}\right\|_{\mathfrak{L}_{1}}<\infty$. Furthermore, $\mathfrak{h}_{n}^{\text {ph }}$ is obviously one-dimensional for any $n \in \mathbb{N}_{0}$. Hence, $\left\|I_{\mathfrak{h}_{n}^{p h}}\right\|_{\mathfrak{L}_{1}}=1$. From (2.29) and $V_{e l}=v_{e l} \otimes I_{\mathfrak{h}^{p h}}$, we obtain the following:

$$
\begin{aligned}
\left\|\left(H_{0}+i\right)^{-1} V_{e l}\left(H_{0}+i\right)^{-1}\right\|_{\mathfrak{L}_{1}} & =\sum_{n \in \mathbb{N}_{0}}\left\|\left(h_{0}^{e l}+n \omega+i\right)^{-1} v_{e l}\left(h_{0}^{e l}+n \omega+i\right)^{-1}\right\|_{\mathfrak{L}_{1}} \\
& \leqslant \sum_{n \in \mathbb{N}_{0}}\left\|\left(h_{0}^{e l}+n \omega+i\right)^{-2}\right\|\left\|v_{e l}\right\|_{\mathfrak{L}_{1}}
\end{aligned}
$$

Since $h_{0}^{e l}$ is bounded, we get:

$$
\left\|\left(h_{0}^{e l}+n \omega+i\right)^{-1}\right\|=\sup _{\lambda \in \sigma\left(h_{0}^{e l}\right)}\left(\sqrt{(\lambda+n \omega)^{2}+1}\right)^{-1} \leqslant c(n+1)^{-1},
$$

for some $c>0$. This immediately implies that $\left\|\left(H_{0}+i\right)^{-1} V_{e l}\left(H_{0}+i\right)^{-1}\right\|_{\mathfrak{L}_{1}}<\infty$.

We are going to handle $\left(H_{0}+i\right)^{-1} V_{p h}\left(H_{0}+i\right)^{-1}$. Let $p_{n}^{p h}$ be the projection from $\mathfrak{h}^{p h}$ onto $\mathfrak{h}_{n}^{p h}$. We have the following:

$$
\begin{aligned}
& \left(H_{0}+i\right)^{-1}\left(\cdot, e_{0}^{S}\right) e_{1}^{S} \otimes b\left(H_{0}+i\right)^{-1} \\
& \quad=\sum_{m, n \in \mathbb{N}_{0}}\left(h_{0}^{e l}+m \omega+i\right)^{-1}\left(\cdot, e_{0}^{S}\right) e_{1}^{S}\left(h_{0}^{e l}+n \omega+i\right)^{-1} \otimes p_{m}^{p h} b p_{n}^{p h} \\
& \quad=\sum_{n \in \mathbb{N}}\left(h_{0}^{e l}+(n-1) \omega+i\right)^{-1}\left(\cdot, e_{0}^{S}\right) e_{1}^{S}\left(h_{0}^{e l}+n \omega+i\right)^{-1} \otimes \sqrt{n} \Upsilon_{n-1}\left\langle\cdot, \Upsilon_{n}\right\rangle
\end{aligned}
$$

From (2.30), we get the following:

$$
\left.\|\left(h_{0}^{e l}+(n-1) \omega+i\right)^{-1}\left(\cdot, e_{0}^{S}\right) e_{1}^{S}\left(h_{0}^{e l}+n \omega+i\right)^{-1}\right) \otimes \sqrt{n} \Upsilon_{n}\left\langle\cdot, \Upsilon_{n}\right\rangle \|_{\mathfrak{L}_{1}} \leqslant c^{2} \frac{\sqrt{n}}{n(n+1)},
$$

$n \in \mathbb{N}$, which yields:

$$
\left\|\left(H_{0}+i\right)^{-1}\left(\cdot, e_{0}^{S}\right) e_{1}^{S} \otimes b\left(H_{0}+i\right)^{-1}\right\|_{\mathfrak{L}_{1}} \leqslant c^{2} \sum_{n \in \mathbb{N}}^{\infty} \frac{\sqrt{n}}{n(n+1)}<\infty .
$$

Since

$$
\left\|\left(H_{0}+i\right)^{-1}\left(\cdot, e_{1}^{S}\right) e_{0}^{S} \otimes b^{*}\left(H_{0}+i\right)^{-1}\right\|_{\mathfrak{L}_{1}}=\left\|\left(H_{0}+i\right)^{-1}\left(\cdot, e_{0}^{S}\right) e_{1}^{S} \otimes b\left(H_{0}+i\right)^{-1}\right\|_{\mathfrak{L}_{1}},
$$

one gets $\left(H_{0}+i\right)^{-1} V_{p h}\left(H_{0}+i\right)^{-1} \in \mathfrak{L}_{1}(\mathfrak{H})$, which completes the proof.

Thus, the $J C L$-model $\mathcal{S}=\left\{H, H_{0}\right\}$ is a $\mathfrak{L}_{1}$-scattering system. Let us recall that $h_{\alpha}^{e l}=$ $-\Delta^{D}+v_{\alpha}, \alpha \in\{l, r\}$, on $\mathfrak{h}_{l}^{e l}=\mathfrak{h}_{r}^{e l}=\ell^{2}(\mathbb{N})$.

Lemma 2.10. Let $\alpha \in\{l, r\}$. We have the following:

$$
\sigma\left(h_{\alpha}^{e l}\right)=\sigma_{a c}\left(h_{\alpha}^{e l}\right)=\left[v_{\alpha}, 4+v_{\alpha}\right] .
$$

The normalized generalized eigenfunctions of $h_{\alpha}^{e l}$ are given by:

$$
g_{\alpha}(x, \lambda)=\pi^{-\frac{1}{2}}\left(1-\left(-\lambda+2+v_{\alpha}\right)^{2} / 4\right)^{-\frac{1}{4}} \sin \left(\arccos \left(\left(-\lambda+2+v_{\alpha}\right) / 2\right) x\right)
$$

for $x \in \mathbb{N}, \lambda \in\left(v_{\alpha}, 4+v_{\alpha}\right)$.

Proof. We prove the absolute continuity of the spectrum by showing that:

$$
\left\{g_{\alpha}(x, \lambda) \mid \lambda \in(-2,2)\right\}
$$

is a complete set of generalized eigenfunctions. Note that it suffices to prove the lemma for

$$
\left(\left(\Delta^{D}+2\right) f\right)(x)=f(x+1)+f(x-1), \quad f(0)=0 .
$$


The lemma then follows by replacing $\lambda$ with $-\lambda+2+v_{\alpha}$. Let $\lambda \in(-2,2)$ and

$$
g_{\Delta^{D}}(x, \lambda)=\pi^{-\frac{1}{2}}\left(1-\lambda^{2} / 4\right)^{-\frac{1}{4}} \sin (\arccos (\lambda / 2) x) .
$$

Note that $g_{\Delta^{D}}(0, \lambda)=0$, when the boundary condition is satisfied. We substitute $\mu=$ $\arccos (\lambda / 2) \in(0, \pi)$, i.e. $\lambda=2 \cos (\mu)$ and obtain the following:

$$
\sin (\mu(x+1))+\sin (\mu(x-1))=2 \sin (\mu x) \cos (\mu),
$$

when $g_{\Delta^{D}}(x, \lambda)$ satisfies the eigenvalue equation. It is obvious that $g_{\Delta^{D}}(\cdot, \lambda) \notin \ell^{2}\left(\mathbb{N}_{0}\right)$ for $\lambda \in$ $(-2,2)$. To complete the proof of the lemma, it remains to show the ortho-normality and the completeness. For the ortho-normality, we have to show that

$$
\sum_{x \in \mathbb{N}} g_{\Delta^{D}}(x, \lambda) g_{\Delta^{D}}(x, \nu)=\delta(\lambda-\nu) .
$$

Let $\psi \in C_{0}^{\infty}((-2,2))$. We use the substitution $\mu=\arccos (\nu / 2)$ and the relation

$$
\sin (\arccos (y))=\left(1-y^{2}\right)^{-\frac{1}{2}}
$$

to obtain the following:

$$
\begin{aligned}
& \int_{-2}^{2} \mathrm{~d} \nu \sum_{x \in \mathbb{N}} g_{\Delta^{D}}(x, \lambda) g_{\Delta^{D}}(x, \nu) \psi(\nu) \\
& =2 \pi^{-1} \int_{0}^{\pi} \mathrm{d} \mu \sum_{x \in \mathbb{N}} \frac{\sin (\mu) \sin (\arccos (\lambda / 2) x) \sin (\mu x)}{(\sin (\mu))^{\frac{1}{2}}(\sin (\arccos (\lambda / 2)))^{\frac{1}{2}}} \psi(2 \cos (\mu)) \\
& =(2 \pi)^{-1} \int_{0}^{\pi} \mathrm{d} \mu \sum_{x \in \mathbb{N}} \frac{(\sin (\mu))^{\frac{1}{2}}}{(\sin (\arccos (\lambda / 2)))^{\frac{1}{2}}}\left(e^{i(\arccos (\lambda / 2)-\mu) x}+\right. \\
& \left.e^{-i(\arccos (\lambda / 2)-\mu) x}-e^{i(\arccos (\lambda / 2)+\mu) x}-e^{-i(\arccos (\lambda / 2)+\mu) x}\right) \psi(2 \cos (\mu)) .
\end{aligned}
$$

Observe that for the Dirichlet kernel:

$$
\sum_{x \in \mathbb{N}_{0}}\left(e^{i x y}+e^{-i x y}\right)-1=2 \pi \delta(y)
$$

when

$$
\begin{aligned}
& \int_{-2}^{2} \mathrm{~d} \nu \sum_{x \in \mathbb{N}} g_{\Delta^{D}}(x, \lambda) g_{\Delta^{D}}(x, \nu) \psi(\nu)= \\
& \int_{0}^{\pi} \mathrm{d} \mu \frac{(\sin (\mu))^{\frac{1}{2}}}{(\sin (\arccos (\lambda / 2)))^{\frac{1}{2}}}(\delta(\arccos (\lambda / 2)-\mu)+\delta(\arccos (\lambda / 2)+\mu)) \psi(2 \cos (\mu))=\psi(\lambda) .
\end{aligned}
$$

In the second equality we use that the summand containing $\delta(\arccos (\lambda / 2)+\mu)$ is zero since both $\arccos (\lambda / 2)>0$ and $\mu>0$. Thus, the generalized eigenfunctions are orthonormal. Finally, using once more the substitution $\mu=\arccos (\nu / 2)$, we obtain the following:

$$
\begin{aligned}
& \int_{-2}^{2} \mathrm{~d} \nu g_{\Delta^{D}}(x, \nu) g_{\Delta^{D}}(y, \nu)= \\
& \int_{-2}^{2} \mathrm{~d} \nu\left(1-(\nu / 2)^{2}\right)^{-\frac{1}{2}} \sin (\arccos (\nu / 2) x) \sin (\arccos (\nu / 2) y)= \\
& 2 \pi^{-1} \int_{0}^{\pi} \mathrm{d} \mu(\sin (\mu))^{-1} \sin (\mu) \sin (\mu x) \sin (\mu y)=\delta_{x y},
\end{aligned}
$$

for $x, y \in \mathbb{N}$, when the family of generalized eigenfunctions is also complete. 
From these two lemmas, we obtain the following corollary that gives us the spectral properties of $H_{0}$.

Proposition 2.11. Let $\mathcal{S}=\left\{H, H_{0}\right\}$ be the JCL-model. Then, $\sigma\left(H_{0}\right)=\sigma_{a c}\left(H_{0}\right) \cup \sigma_{p p}\left(H_{0}\right)$, where

$$
\sigma_{a c}\left(H_{0}\right)=\bigcup_{n \in \mathbb{N}_{0}}\left[v_{l}+n \omega, v_{l}+4+n \omega\right] \cup\left[v_{r}+n \omega, v_{r}+4+n \omega\right]
$$

and

$$
\sigma_{p p}\left(H_{0}\right)=\bigcup_{n \in \mathbb{N}_{0}}\left\{\lambda_{j}^{S}+n \omega: j=0,1\right\} .
$$

The eigenvectors are given by $\widetilde{g}(m, n)=e_{m}^{S} \otimes \Upsilon_{n}, m=0,1, n \in \mathbb{N}_{0}$. The generalized eigenfunctions are given by $\widetilde{g}_{\alpha}(\cdot, \lambda, n)=g_{\alpha}(\cdot, \lambda-n \omega) \otimes \Upsilon_{n}$ for $\lambda \in \sigma_{a c}\left(H_{0}\right), n \in \mathbb{N}_{0}, \alpha \in\{l, r\}$.

Proof. It is well known (see e.g. [15]) that for two self-adjoint operators $A$ and $B$ with $\sigma_{s c}(A)=$ $\sigma_{s c}(B)=\emptyset$, we have $\sigma_{s c}(A \otimes 1+1 \otimes B)=\emptyset$,

$$
\sigma_{a c}(A \otimes 1+1 \otimes B)=\left(\sigma_{a c}(A)+\sigma(B)\right) \cup\left(\sigma(A)+\sigma_{a c}(B)\right)
$$

and

$$
\sigma_{p p}(A \otimes 1+1 \otimes B)=\sigma_{p p}(A)+\sigma_{p p}(B) .
$$

Furthermore, if $\psi_{A}\left(\lambda_{A}\right)$ and $\psi_{B}\left(\lambda_{B}\right)$ are (generalized) eigenfunctions of $A$ and $B$, respectively, then $\psi_{A}\left(\lambda_{A}\right) \otimes \psi_{B}\left(\lambda_{B}\right)$ is a (generalized) eigenfunction of $A \otimes I+I \otimes B$ for the (generalized) eigenvalue $\lambda_{A}+\lambda_{B}$.

The lemma follows now with $A=h_{0}^{e l}$ and $B=h^{p h}$ using Lemmata 2.10 and (2.14) and the fact that $h_{S}$ has eigenvectors $\left\{e_{0}^{S}, e_{1}^{S}\right\}$ with eigenvalues $\left\{\lambda_{0}^{S}, \lambda_{1}^{S}=\lambda_{0}^{S}+\varepsilon\right\}$.

\subsection{Spectral representation}

For the convenience of the reader, we define here what we mean under a spectral representation of the absolutely continuous part $K_{0}^{a c}$ of a self-adjoint operator $K_{0}$ on a separable Hilbert space $\mathfrak{K}$. Let $\mathfrak{k}$ be an auxiliary separable Hilbert space. We consider the Hilbert space $L^{2}(\mathbb{R}, d \lambda, \mathfrak{k})$. By $\mathcal{M}$, we define the multiplication operator induced by the independent variable $\lambda$ in $L^{2}(\mathbb{R}, d \lambda, \mathfrak{k})$. Let $\Phi: \mathfrak{K}^{a c}\left(K_{0}\right) \longrightarrow L^{2}(\mathbb{R}, d \lambda, \mathfrak{k})$ be an isometry acting from $\mathfrak{K}^{a c}\left(K_{0}\right)$ into $L^{2}(\mathbb{R}, d \lambda, \mathfrak{k})$ such that: $\Phi \operatorname{dom}\left(K_{0}^{a c}\right) \subseteq \operatorname{dom}(\mathcal{M})$ and

$$
\mathcal{M} \Phi f=\Phi K_{0}^{a c} f, \quad f \in \operatorname{dom}\left(K_{0}^{a c}\right) .
$$

Obviously, the orthogonal projection $P:=\Phi \Phi^{*}$ commutes with $\mathcal{M}$ which yields the existence of a measurable family, $\{P(\lambda)\}_{\lambda \in \mathbb{R}}$, such that:

$$
(P \widehat{f})(\lambda)=P(\lambda) \widehat{f}(\lambda), \quad \widehat{f} \in L^{2}(\mathbb{R}, \lambda, \mathfrak{k}) .
$$

We set $L^{2}(\mathbb{R}, d \lambda, \mathfrak{k}(\lambda)):=P L^{2}(\mathbb{R}, \lambda, \mathfrak{k}), \mathfrak{k}(\lambda):=P(\lambda) \mathfrak{k}$, and call the triplet

$$
\Pi\left(K_{0}^{a c}\right):=\left\{L^{2}(\mathbb{R}, d \lambda, \mathfrak{k}(\lambda)), \mathcal{M}, \Phi\right\}
$$

a spectral representation of $K_{0}^{a c}$. If $\left\{L^{2}(\mathbb{R}, d \lambda, \mathfrak{k}(\lambda)), \mathcal{M}, \Phi\right\}$ is a spectral representation of $K^{a c}$, then $K^{a c}$ is unitarily equivalent $\mathcal{M}_{0}:=\mathcal{M} \uparrow L^{2}(\mathbb{R}, d \lambda, \mathfrak{k}(\lambda))$. Indeed, one has $\Phi K_{0}^{a c} \Phi^{*}=\mathcal{M}_{0}$. The function $\xi_{K_{0}}^{a c}(\lambda):=\operatorname{dom}(\mathfrak{k}(\lambda)), \lambda \in \mathbb{R}$, is called the spectral multiplicity function of $K_{0}^{a c}$. Notice that $0 \leqslant \xi_{K_{0}}^{a c}(\lambda) \leqslant \infty$ for $\lambda \in \mathbb{R}$.

For $\alpha \in\{l, r\}$, the generalized eigenfunctions of $h_{\alpha}^{e l}$ define generalized Fourier transforms by $\phi_{\alpha}^{e l}: \mathfrak{h}_{\alpha}^{e l}=\mathfrak{h}_{\alpha}^{e l, a c}\left(h_{\alpha}^{e l}\right) \rightarrow L^{2}\left(\left[v_{\alpha}, v_{\alpha}+4\right]\right)$ and

$$
\left(\phi_{\alpha}^{e l} f_{\alpha}\right)(\lambda)=\sum_{x \in \mathbb{N}_{0}} g_{\alpha}(x, \lambda) f_{\alpha}(x), \quad f_{\alpha} \in \mathfrak{h}_{\alpha}^{e l} .
$$


We then set:

$$
\mathfrak{h}_{\alpha}^{e l}(\lambda):= \begin{cases}\mathbb{C} & \lambda \in\left[v_{\alpha}, v_{\alpha}+4\right] \\ 0 & \lambda \in \mathbb{R} \backslash\left[v_{\alpha}, v_{\alpha}+4\right] .\end{cases}
$$

One can easily verify that $\Pi\left(h_{\alpha}^{e l}\right)=\left\{L^{2}\left(\mathbb{R}, d \lambda, \mathfrak{h}_{\alpha}^{\text {el }}(\lambda)\right), \mathcal{M}, \phi_{\alpha}^{\text {el }}\right\}$ is a spectral representation of $h_{\alpha}^{e l}=h_{\alpha}^{e l, a c}, \alpha=l, r$, where we always assumed implicitly that $\left(\phi_{\alpha}^{e l} f_{\alpha}\right)(\lambda)=0$ for $\lambda \in \mathbb{R} \backslash$ $\left[v_{\alpha}, v_{\alpha}+4\right]$. Setting:

$$
\mathfrak{h}^{e l}(\lambda):=\underset{\mathfrak{h}_{r}^{e l}(\lambda)}{\mathfrak{h}_{l}^{e l}(\lambda)} \subseteq \mathbb{C}^{2}, \quad \lambda \in \mathbb{R}
$$

and introducing the map:

$$
\phi^{e l}: \mathfrak{h}^{e l, a c}\left(h_{0}^{e l}\right)=\underset{\mathfrak{h}_{r}^{e l}}{\bigoplus} \underset{\mathfrak{h}_{l}^{e l}}{\bigoplus} \longrightarrow L^{2}\left(\mathbb{R}, d \lambda, \mathfrak{h}^{e l}(\lambda)\right),
$$

defined by:

$$
\phi^{e l} f:=\left(\begin{array}{l}
\phi_{l}^{e l} f_{l} \\
\phi_{r}^{e l} f_{r}
\end{array}\right), \quad \text { where } f:=\left(\begin{array}{c}
f_{l} \\
f_{r}
\end{array}\right)
$$

we obtain a spectral representation $\Pi\left(h_{0}^{e l, a c}\right)=\left\{L^{2}\left(\mathbb{R}, d \lambda, \mathfrak{h}^{e l}(\lambda)\right), \mathcal{M}, \phi^{e l}\right\}$ of the absolutely continuous part $h_{0}^{e l, a c}=h_{l}^{e l} \oplus h_{r}^{e l}$ of $h_{0}^{e l}$. One easily verifies that $0 \leqslant \xi_{h_{0}^{e l}}^{a c}(\lambda) \leqslant 2$ for $\lambda \in \mathbb{R}$. Introducing:

$$
\lambda_{\min }^{e l}:=\min \left\{v_{l}, v_{r}\right\} \quad \text { and } \quad \lambda_{\max }^{e l}:=\max \left\{v_{l}+4, v_{r}+4\right\},
$$

one easily verifies that $\xi_{h_{0}^{e l}}^{a c}(\lambda)=0$ for $\lambda \in \mathbb{R} \backslash\left[\lambda_{\min }^{e l}, \lambda_{\max }^{e l}\right]$.

Notice, if $v_{r}+4 \leqslant v_{l}$, then

$$
\mathfrak{h}^{e l}(\lambda)= \begin{cases}\mathbb{C}, & \lambda \in\left[v_{r}, v_{r}+4\right] \cup\left[v_{l}, v_{l}+4\right], \\ \{0\}, & \text { otherwise }\end{cases}
$$

which shows that $h_{0}^{e l}$ has a simple spectrum. In particular, it holds $\xi_{h_{0}^{e l}}^{a c}(\lambda)=1$ for $\lambda \in\left[v_{r}, v_{r}+\right.$ 4] $\cup\left[v_{l}, v_{l}+4\right]$ and otherwise $\xi_{h_{0}^{e l}}^{a c}(\lambda)=0$.

Let us introduce the Hilbert space $\mathfrak{h}:=l^{2}\left(\mathbb{N}_{0}, \mathbb{C}^{2}\right)=\bigoplus_{n \in \mathbb{N}_{0}} \mathfrak{h}_{n}, \mathfrak{h}_{n}:=\mathbb{C}^{2}, n \in \mathbb{N}_{0}$. Regarding $\mathfrak{h}^{e l}(\lambda-n \omega)$ as a subspace of $\mathfrak{h}_{n}$, one regards:

$$
\mathfrak{h}(\lambda):=\bigoplus_{n \in \mathbb{N}_{0}} \mathfrak{h}_{n}(\lambda), \quad \mathfrak{h}_{n}(\lambda):=\mathfrak{h}^{e l}(\lambda-n \omega), \quad \lambda \in \mathbb{R},
$$

as a measurable family of subspaces in $\mathfrak{h}$. Notice that $0 \leqslant \operatorname{dim}(\mathfrak{h}(\lambda))<\infty, \lambda \in \mathbb{R}$. We consider the Hilbert space $L^{2}(\mathbb{R}, d \lambda, \mathfrak{h}(\lambda))$.

Furthermore, we introduce the isometric map $\Phi: \mathfrak{H}\left(H_{0}^{a c}\right) \longrightarrow L^{2}(\mathbb{R}, d \lambda, \mathfrak{h}(\lambda))$ defined by

$$
(\Phi f)(\lambda)=\bigoplus_{n \in \mathbb{N}_{0}}\left(\begin{array}{c}
\left(\phi_{l}^{e l} f_{l}(n)\right)(\lambda-n \omega) \\
\left(\phi_{r}^{\text {el }} f_{r}(n)\right)(\lambda-n \omega)
\end{array}\right), \quad \lambda \in \mathbb{R},
$$

where

$$
\bigoplus_{n \in \mathbb{N}_{0}}\left(\begin{array}{c}
f_{l}(n) \\
f_{r}(n)
\end{array}\right) \in \bigoplus_{n \in \mathbb{N}_{0}} \mathfrak{h}^{e l, a c}\left(h_{0}^{e l}\right) \otimes \mathfrak{h}_{n}^{p h}=\bigoplus_{n \in \mathbb{N}}\left(\begin{array}{c}
\mathfrak{h}_{l}^{e l} \otimes h_{n}^{p h} \\
\oplus \\
\mathfrak{h}_{r}^{e l} \otimes h_{n}^{p h}
\end{array}\right)
$$

where $\mathfrak{h}_{p h}=\bigoplus_{n \in \mathbb{N}_{0}} \mathfrak{h}_{n}^{p h}$ and $\mathfrak{h}_{n}^{p h}$ is the subspace spanned by the eigenvectors $\Upsilon_{n}$ of $h^{p h}$. One easily verifies that $\Phi$ is an isometry acting from $\mathfrak{H}^{a c}\left(H_{0}^{a c}\right)$ onto $L^{2}(\mathbb{R}, d \lambda, \mathfrak{h}(\lambda))$. 
Lemma 2.12. The triplet $\left\{L^{2}(\mathbb{R}, d \lambda, \mathfrak{h}(\lambda)), \mathcal{M}, \Phi\right\}$ forms a spectral representation of $H_{0}^{a c}$, that is, $\Pi\left(H_{0}^{a c}\right)=\left\{L^{2}(\mathbb{R}, d \lambda, \mathfrak{h}(\lambda)), \mathcal{M}, \Phi\right\}$ where there is a constant $d \in \mathbb{N}_{0}$ such that $0 \leqslant \xi_{H_{0}}^{a c}(\lambda) \leqslant$ $2 d_{\max }$ for $\lambda \in \mathbb{R}$ where $d_{\max }:=\frac{\lambda_{\max }^{e l}-\lambda_{\min }^{e l}}{\omega}$ and $\lambda_{\max }^{e l}$ and $\lambda_{\min }^{e l}$ are given by (2.36).

Proof. It remains to be shown that $\Phi$ transforms $H_{0}^{a c}$ into the multiplication operator $\mathcal{M}$. We have

$$
H_{0}^{a c} f=\bigoplus_{n \in \mathbb{N}_{0}}\left(\begin{array}{c}
\left(h_{l}^{e l} f_{l}\right)(n)+n \omega f_{l}(n) \\
\left(h_{r}^{e l} f_{r}\right)(n)+n \omega f_{r}(n)
\end{array}\right)
$$

which yields the following:

$$
\begin{aligned}
\left(\Phi H_{0}^{a c} f\right)(\lambda) & =\bigoplus_{n \in \mathbb{N}_{0}}\left(\begin{array}{c}
\left(\phi_{l}^{e l}\left(h_{l}^{e l} f_{l}\right)(n)\right)(\lambda-n \omega)+n \omega\left(\phi_{l}^{e l} f_{l}(n)\right)(\lambda-n \omega) \\
\left(\phi_{r}^{e l}\left(h_{r}^{e l} f_{r}\right)(n)\right)(\lambda-n \omega)+n \omega\left(\phi_{r}^{e l} f_{r}(n)\right)(\lambda-n \omega)
\end{array}\right) \\
& =\bigoplus_{n \in \mathbb{N}_{0}}\left(\begin{array}{c}
\lambda\left(\phi_{l}^{e l} f_{l}(n)\right)(\lambda-n \omega) \\
\lambda\left(\phi_{r}^{e l} f_{r}(n)\right)(\lambda-n \omega)
\end{array}\right)=(\mathcal{M} \Phi f)(\lambda), \quad \lambda \in \mathbb{R} .
\end{aligned}
$$

which proves the desired property.

One easily checks that $\mathfrak{h}(\lambda)$ might only be only non-trivial if $\lambda-n \omega \in\left[\lambda_{\min }^{e l}, \lambda_{\max }^{e l}\right]$. Hence, we obtain that $\mathfrak{h}(\lambda)$ is non-trivial if the condition:

$$
\frac{\lambda-\lambda_{\max }^{e l}}{\omega} \leqslant n \leqslant \frac{\lambda-\lambda_{\min }^{e l}}{\omega}
$$

is satisfied. Hence,

$$
0 \leqslant \xi_{H_{0}}^{a c}(\lambda) \leqslant 2 \operatorname{card}\left\{n \in \mathbb{N}_{0}: \frac{\lambda-\lambda_{\text {max }}^{e l}}{\omega} \leqslant n \leqslant \frac{\lambda-\lambda_{\text {min }}^{e l}}{\omega}\right\}, \quad \lambda \in \mathbb{R} .
$$

or

$$
0 \leqslant \xi_{H_{0}}^{a c}(\lambda) \leqslant 2 \operatorname{card}\left\{n \in \mathbb{N}_{0}: 0 \leqslant n \leqslant \frac{\lambda_{\max }^{e l}-\lambda_{\max }^{e l}}{\omega}\right\}, \quad \lambda \in \mathbb{R} .
$$

Hence $0 \leqslant \xi_{H_{0}}^{a c}(\lambda) \leqslant d_{\max }$ for $\lambda \in \mathbb{R}$.

In the following we denote the orthogonal projection from $\mathfrak{h}(\lambda)$ onto $\mathfrak{h}_{n}(\lambda)$ by $P_{n}(\lambda)$, $\lambda \in \mathbb{R}$, cf (2.37). Since $\mathfrak{h}(\lambda)=\bigoplus_{n \in \mathbb{N}_{0}} \mathfrak{h}_{n}(\lambda)$ we have $I_{\mathfrak{h}(\lambda)}=\sum_{n \in \mathbb{N}_{0}} P_{n}(\lambda), \lambda \in \mathbb{R}$. Further, we introduce the following subspaces:

$$
\mathfrak{h}_{n_{\alpha}}(\lambda):=\mathfrak{h}_{\alpha}^{e l}(\lambda-n \omega), \quad \lambda \in \mathbb{R}, \quad n \in \mathbb{N}_{0}
$$

Notice that:

$$
\mathfrak{h}_{n}(\lambda)=\bigoplus_{\alpha \in\{l, r\}} \mathfrak{h}_{n_{\alpha}}(\lambda), \quad \lambda \in \mathbb{R}, \quad n \in \mathbb{N}_{0}
$$

By $P_{n_{\alpha}}(\lambda)$ we denote the orthogonal projection from $\mathfrak{h}(\lambda)$ onto $\mathfrak{h}_{n_{\alpha}}(\lambda), \lambda \in \mathbb{R}$. Clearly, we have $P_{n}(\lambda)=\sum_{\alpha \in\{l, r\}} P_{n_{\alpha}}(\lambda), \lambda \in \mathbb{R}$.

Example 2.13. In general, the direct integral $\Pi\left(H_{0}^{a c}\right)$ can be very complicated, in particular, the structure of $\mathfrak{h}(\lambda)$ given by (2.37) is difficult to analyze. However, there are interesting simple cases:

(i) Let $v=v_{l}=v_{r}$ and $4 \leqslant \omega$. In this case we have $\mathfrak{h}^{e l}(\lambda)=\mathbb{C}^{2}$ for $[v, v+4]$ and

$$
\mathfrak{h}(\lambda)= \begin{cases}\mathbb{C}^{2}, & \lambda \in[v+n \omega, v+n \omega+4], \quad n \in \mathbb{N}_{0}, \\ \{0\}, & \text { otherwise. }\end{cases}
$$


(ii) Let $v_{r}=0, v_{l}=4, \omega_{0}=4$. Then

$$
\mathfrak{h}(\lambda)= \begin{cases}\mathfrak{h}_{r}^{e l}(\lambda)=\mathbb{C}, & \lambda \in[0,4), \\ \mathfrak{h}_{l r}^{e l}(\lambda)=\mathbb{C}^{2}, & \lambda \in[4,8), \\ \mathfrak{h}_{r l}^{e l}(\lambda)=\mathbb{C}^{2}, & \lambda \in[8,12), \\ \cdots & \end{cases}
$$

where

$$
\mathfrak{h}_{\alpha \alpha^{\prime}}^{e l}(\lambda)=\underset{\substack{\mathfrak{h}_{\alpha}^{e l}(\lambda) \\ \mathfrak{h}_{\alpha^{\prime}}^{e l}(\lambda)}}{\bigoplus}, \quad \alpha, \alpha^{\prime} \in\{l, r\}, \quad \alpha \neq \alpha^{\prime} .
$$

Hence, $\operatorname{dim}(\mathfrak{h}(\lambda))=2$ for $\lambda \geqslant 4$.

Let $Z$ be a bounded operator acting on $\mathfrak{H}^{a c}\left(H_{0}\right)$ and commuting with $H_{0}^{a c}$. Since $Z$ commutes with $H_{0}^{a c}$ there is a measurable family $\{Z(\lambda)\}_{\lambda \in \mathbb{R}}$ of bounded operators acting on $\mathfrak{h}(\lambda)$ such that $Z$ is unitarily equivalent to the multiplication operator induced by $\{Z(\lambda)\}_{\lambda \in \mathbb{R}}$ in $\Pi\left(H_{0}^{a c}\right)$. We then set:

$$
Z_{m_{\alpha} n_{\varkappa}}(\lambda):=P_{m_{\alpha}}(\lambda) Z(\lambda)\left\lceil\mathfrak{h}_{n_{\varkappa}}(\lambda), \quad \lambda \in \mathbb{R}, \quad m, n \in \mathbb{N}_{0}, \quad \alpha, \varkappa \in\{l, r\} .\right.
$$

Let $Z_{m_{\alpha} n_{\varkappa}}:=P_{m_{\alpha}} Z P_{n_{\varkappa}}$ where $P_{m_{\alpha}}$ is the orthogonal projection from $\mathfrak{H}$ onto $\mathfrak{H}_{m_{\alpha}} \subseteq \mathfrak{H}^{a c}\left(H_{0}\right)$, cf. (2.18). Clearly, the multiplication operator induced $\left\{Z_{m_{\alpha} n_{\varkappa}}(\lambda)\right\}_{\lambda \in \mathbb{R}}$ in $\Pi\left(H_{0}^{a c}\right)$ is unitarily equivalent to $Z_{m_{\alpha} n_{\varkappa}}$.

Since, by Lemma $2.12, \mathfrak{h}(\lambda)$ is a finite dimensional space, the operators $Z(\lambda)$ are finite dimensional ones and we can introduce the following quantity:

$$
\sigma_{m_{\alpha} n_{\varkappa}}(\lambda):=\operatorname{tr}\left(Z_{m_{\alpha} n_{\varkappa}}(\lambda)^{*} Z_{m_{\alpha} n_{\varkappa}}(\lambda)\right), \quad \lambda \in \mathbb{R}, \quad m, n \in \mathbb{N}_{0}, \quad \alpha, \varkappa \in\{l, r\} .
$$

Lemma 2.14. Let $H_{0}$ be the self-adjoint operator defined by (2.15) on $\mathfrak{H}$. Furthermore, let $Z$ be a bounded operator on $\mathfrak{H}^{a c}\left(H_{0}\right)$ commuting with $H_{0}^{a c}$

(i) Let $\Gamma$ be a conjugation on $\mathfrak{H}$, cf. Section 2.3. If $\Gamma$ commutes with $H_{0}$ and $P_{n_{\alpha}}, n \in \mathbb{N}_{0}$, $\alpha \in\{l, r\}$ and $\Gamma Z \Gamma=Z^{*}$ holds, then $\sigma_{m_{\alpha} n_{\varkappa}}(\lambda)=\sigma_{n_{\varkappa} m_{\alpha}}(\lambda), \lambda \in \mathbb{R}$.

(ii) Let $U$ be a mirror symmetry on $\mathfrak{H}$. If $U$ commutes with $H_{0}$ and $Z$, then $\sigma_{m_{\alpha} n_{\varkappa}}(\lambda)=$ $\sigma_{m_{\alpha^{\prime}} n_{\varkappa^{\prime}}}(\lambda), \lambda \in \mathbb{R}, m, n \in \mathbb{N}_{0}, \alpha, \alpha^{\prime}, \varkappa, \varkappa^{\prime} \in\{l, r\}, \alpha \neq \alpha^{\prime}, \varkappa \neq \varkappa^{\prime}$.

Proof. (i) Since $\Gamma$ commutes with $H_{0}$ the conjugation $\Gamma$ is reduce by $\mathfrak{H}^{a c}\left(H_{0}\right)$. So without loss of generality, we assume that $\Gamma$ acts on $\mathfrak{H}^{a c}\left(H_{0}\right)$. We set $\Gamma_{n_{\alpha}}:=\Gamma\left\lceil\mathfrak{H}_{n_{\alpha}}\right.$. Notice that:

$$
\Gamma=\bigoplus_{n \in \mathbb{N}_{0}, \alpha \in\{l, r\}} \Gamma_{n_{\alpha}}
$$

There is a measurable family $\{\Gamma(\lambda)\}_{\lambda \in \mathbb{R}}$ of conjugations such that the multiplication operator induced by $\{\Gamma(\lambda)\}_{\lambda \in \mathbb{R}}$ in $\Pi\left(H_{0}^{a c}\right)$ is unitarily equivalent to $\Gamma$. Moreover, since $\Gamma$ commutes with $P_{n_{\alpha}}$, we see that the multiplication operator induced by the measurable family:

$$
\Gamma_{n_{\alpha}}(\lambda):=\Gamma(\lambda) \uparrow \mathfrak{h}_{n_{\alpha}}(\lambda), \quad \lambda \in \mathbb{R}, \quad m \in \mathbb{N}_{0}, \quad \alpha \in\{l, r\}
$$

is unitarily equivalent to $\Gamma_{n_{\alpha}}$. Using $\Gamma Z \Gamma=Z^{*}$ we get $\Gamma_{m_{\alpha}} Z_{m_{\alpha} n_{\varkappa}} \Gamma_{n_{\varkappa}}=Z_{n_{\varkappa} m_{\alpha}}^{*}$. Hence,

$$
\Gamma_{m_{\alpha}}(\lambda) Z_{m_{\alpha} n_{\varkappa}}(\lambda) \Gamma_{n_{\varkappa}}(\lambda)=Z_{n_{\varkappa} m_{\alpha}}(\lambda)^{*}, \quad \lambda \in \mathbb{R} .
$$

If $X$ is a trace class operator, then $\operatorname{tr}(\Gamma X \Gamma)=\overline{\operatorname{tr}(X)}$. Using that we find

$$
\frac{\sigma_{m_{\alpha} n_{\varkappa}}(\lambda)=\overline{\operatorname{tr}\left(\Gamma_{n_{\varkappa}}(\lambda) Z_{m_{\alpha} n_{\varkappa}}(\lambda) * Z_{m_{\alpha} n_{\varkappa}}(\lambda) \Gamma_{n_{\varkappa}}(\lambda)\right)}}{\operatorname{tr}\left(\Gamma_{n_{\varkappa}}(\lambda) Z_{m_{\alpha} n_{\varkappa}}(\lambda) * \Gamma_{m_{\alpha}} \Gamma_{m_{\alpha}} Z_{m_{\alpha} n_{\varkappa}}(\lambda) \Gamma_{n_{\varkappa}}(\lambda)\right)}=
$$


From (2.39), we obtain the following:

$$
\sigma_{m_{\alpha} n_{\varkappa}}(\lambda)=\overline{\operatorname{tr}\left(Z_{n_{\varkappa} m_{\alpha}}(\lambda) Z_{n_{\varkappa} m_{\alpha}}(\lambda)^{*}\right)}=\sigma_{n_{\varkappa} m_{\alpha}}(\lambda), \quad \lambda \in \mathbb{R}
$$

which proves (i).

(ii) Again, without loss of generality we can assume that $U$ acts only $\mathfrak{H}^{a c}\left(H_{0}\right)$. Since $U$ commutes with $H_{0}$, there is a measurable family $\{U(\lambda)\}_{\lambda \in \mathbb{R}}$ of unitary operators acting on $\mathfrak{h}(\lambda)$ such that the multiplication operator induced by $\{U(\lambda)\}_{\lambda \in \mathbb{R}}$ is unitarily equivalent to $U$. Since $U \mathfrak{H}_{n_{\alpha}}=\mathfrak{H}_{n_{\alpha^{\prime}}}$ we have $U(\lambda) \mathfrak{h}_{n_{\alpha}}(\lambda)=\mathfrak{h}_{n_{\alpha^{\prime}}}(\lambda), \lambda \in \mathbb{R}$. Hence,

$$
\begin{gathered}
\sigma_{m_{\alpha} n_{\varkappa}}(\lambda)=\operatorname{tr}\left(U(\lambda) Z_{m_{\alpha} n_{\varkappa}}(\lambda)^{*} Z_{m_{\alpha} n_{\varkappa}}(\lambda) U(\lambda)^{*}\right)= \\
\quad \operatorname{tr}\left(U(\lambda) Z_{m_{\alpha}, n_{\varkappa}}(\lambda)^{*} U(\lambda)^{*} U(\lambda) Z_{m_{\alpha}, n_{\varkappa}}(\lambda) U(\lambda)^{*}\right) .
\end{gathered}
$$

Hence,

$$
\sigma_{m_{\alpha} n_{\varkappa}}(\lambda)=\operatorname{tr}\left(P_{n_{\varkappa^{\prime}}} U(\lambda) Z(\lambda)^{*} U(\lambda)^{*} P_{m_{\alpha^{\prime}}}(\lambda) U(\lambda) Z(\lambda) U(\lambda)^{*} P_{n_{\varkappa^{\prime}}}(\lambda)\right) .
$$

Since $U$ commutes with $Z$, we find that:

$$
\sigma_{m_{\alpha} n_{\varkappa}}(\lambda)=\operatorname{tr}\left(P_{n_{\varkappa^{\prime}}} Z(\lambda)^{*} P_{m_{\alpha^{\prime}}}(\lambda) Z(\lambda) P_{n_{\varkappa^{\prime}}}(\lambda)\right)=\sigma_{m_{\alpha^{\prime}} n_{\varkappa^{\prime}}}(\lambda), \quad \lambda \in \mathbb{R},
$$

which proves (ii).

\subsection{Spectral properties of $H$ : second part}

Since we have full information for the spectral properties of $H_{0}$, we can use this to show that $H$ has no singular continuous spectrum. Crucial for that is the following lemma: with the help of [6, Cor. IV.15.19], which establishes existence and completeness of wave operators and absence of singular continuous spectrum through a time-falloff method. We cite it as a Lemma for convenience, with slight simplifications that suffice for our purposes.

Lemma 2.15 ( [6, Corollary IV.15.19]). Let $\left\{H_{0}, H\right\}$ be a scattering system and let $\Lambda$ be a closed countable set. Let $F_{+}$and $F_{-}$be two self-adjoint operators such that $F_{+}+F_{-}=P_{H_{0}}^{a c}$ and

$$
s-\lim _{t \rightarrow \infty} e^{\mp i t H_{0}} F_{ \pm} e^{ \pm i t H_{0}}=0 .
$$

If $(H-i)^{-1}-\left(H_{0}-i\right)^{-1} \in \mathfrak{L}_{\infty}(\mathfrak{H}),\left(1-P_{H_{0}}^{a c}\right) \gamma\left(H_{0}\right) \in \mathfrak{L}_{\infty}(\mathfrak{H})$, and

$$
\left|\int_{0}^{ \pm \infty} \mathrm{d} t\left\|\left(\left(H_{0}-i\right)^{-1}-(H-i)^{-1}\right) e^{-i t H_{0}} \gamma\left(H_{0}\right) F_{ \pm}\right\|\right|<\infty
$$

for all $\gamma \in C_{0}^{\infty}(\mathbb{R} \backslash \Lambda)$, then $W_{ \pm}\left(H, H_{0}\right)$ exist and are complete and $\sigma_{s c}(H)=\sigma_{s c}\left(H_{0}\right)=\emptyset$. Furthermore, each eigenvalue of $H$ and $H_{0}$ in $\mathbb{R} \backslash \Lambda$ is of finite multiplicity and these eigenvalues accumulate the most at points of $\Lambda$ or at $\pm \infty$.

We already know that the wave operators exist and are complete since the resolvent difference is trace class. Hence, we need Lemma 2.15 only to prove the following proposition.

Proposition 2.16. The Hamiltonian $H$ defined by (2.17) has no singular continuous spectrum, that $i s, \sigma_{s c}(H)=\emptyset$.

Proof. At first we have to construct the operators $F_{ \pm}$. To this end, let $\mathcal{F}: L^{2}(\mathbb{R}) \rightarrow L^{2}(\mathbb{R})$ be the usual Fourier transform, i.e

$$
(\mathcal{F} f)(\mu):=\widehat{f}(\mu):=\frac{1}{\sqrt{2 \pi}} \int_{\mathbb{R}} e^{-i \mu x} f(x) d x, \quad f \in L^{2}(\mathbb{R}, d x), \quad \mu \in \mathbb{R} .
$$

Further, let $\Pi_{ \pm}$be the orthogonal projection onto $L^{2}\left(\mathbb{R}_{ \pm}\right)$in $L^{2}(\mathbb{R})$. We set:

$$
F_{ \pm}=\Phi^{*} \mathcal{F} \Pi_{ \pm} \mathcal{F}^{*} \Phi
$$


where $\Phi$ is given by (2.38). We immediately obtain $F_{-}+F_{+}=P_{a c}\left(H_{0}\right)$. We still have to show that:

$$
s-\lim _{t \rightarrow \infty}\left\|e^{\mp i t H_{0}} \Phi^{*} \mathcal{F} \Pi_{ \pm} \mathcal{F}^{*} \Phi e^{ \pm i t H_{0}} f\right\|=0
$$

for $f \in \mathfrak{H}^{a c}\left(H_{0}\right)$. We prove the relation only for $F_{+}$since the proof for $F_{-}$is essentially identical. We have the following:

$$
\left(\Pi_{+} \mathcal{F}^{*} \Phi e^{i t H_{0}} f\right)(x)=(2 \pi)^{-\frac{1}{2}} \chi_{\mathbb{R}_{+}}(x) \int_{\mathbb{R}} \mathrm{d} \mu e^{i(x+t) \mu} \widehat{f}(\mu)=\chi_{\mathbb{R}_{+}}(x) \psi(x+t)
$$

with $\psi=\mathcal{F} \hat{f}$. Now,

$$
\begin{aligned}
& \left\|e^{-i t H_{0}} \Phi^{*} \mathcal{F} \Pi_{+} \mathcal{F}^{*} \Phi e^{i t H_{0}} f\right\|^{2}= \\
& \quad\left\|\Pi_{+} \mathcal{F}^{*} \Phi e^{i t H_{0}} f\right\|^{2}=\int_{\mathbb{R}_{+}} \mathrm{d} x|\psi(x+t)|^{2}=\int_{t}^{\infty} \mathrm{d} x|\psi(x)|^{2} \stackrel{t \rightarrow \infty}{\longrightarrow} 0 .
\end{aligned}
$$

Concerning the compactness condition, we already know that $(H-i)^{-1}-\left(H_{0}-i\right)^{-1} \in \mathfrak{L}_{1}(\mathfrak{H}) \subset$ $\mathfrak{L}_{\infty}(\mathfrak{H})$ from Proposition 2.9. Let

$$
\Lambda:=\bigcup_{n \in \mathbb{N}_{0}}\left\{v_{l}+n \omega, v_{r}+n \omega, v_{l}+4+n \omega, v_{r}+4+n \omega\right\}
$$

which is closed and countable. We know from Corollary 2.11 that $H_{0}$ has no singular continuous spectrum and the eigenvalues are of finite multiplicity. It follows then that $\left(1-P_{a c}\left(H_{0}\right)\right) \gamma\left(H_{0}\right)$ is compact for every $\gamma \in C_{0}^{\infty}(\mathbb{R} \backslash \Lambda)$. The remaining assumption of Lemma 2.15 is as follows:

$$
\left|\int_{0}^{ \pm \infty} \mathrm{d} t\left\|\left((H-i)^{-1}-\left(H_{0}-i\right)^{-1}\right) \gamma\left(H_{0}\right) e^{-i t H_{0}} F_{ \pm}\right\|\right|<\infty
$$

If we can prove this, then we immediately obtain that $H$ has no singular continuous spectrum. Now $(H-i)^{-1}-\left(H_{0}-i\right)^{-1}=(H-i)^{-1}\left(V_{e l}+V_{p h}\right)\left(H_{0}-i\right)^{-1}$. But $(H-i)^{-1}$ is bounded,

$$
\operatorname{ran}\left(F_{ \pm}\right) \subset \mathfrak{H}^{a c}\left(H_{0}\right)=\left(\mathfrak{h}_{l}^{e l} \oplus \mathfrak{h}_{r}^{e l}\right) \otimes \mathfrak{h}^{p h}
$$

and $V_{p h} P^{a c}\left(H_{0}\right)=0$. Also, $V_{e l}=v_{e l} \otimes I_{\mathfrak{h}^{p h}}$ and

$$
\operatorname{ker}\left(v_{e l}\right)^{\perp} \subset \mathbb{C} \delta_{1}^{l} \oplus \mathfrak{h}_{S} \oplus \mathbb{C} \delta_{1}^{r}
$$

Hence, it suffices to prove:

$$
\left|\int_{0}^{ \pm \infty} \mathrm{d} t\left\|P_{1}^{\alpha}\left(H_{0}-i\right)^{-1} \gamma\left(H_{0}\right) e^{-i t H_{0}} F_{ \pm}\right\|\right|<\infty
$$

$\alpha \in\{l, r\}$, where $P_{1}^{\alpha}=p_{1}^{\alpha} \otimes I_{\mathfrak{h}^{p h}}$ and $p_{1}^{\alpha}$ is the orthogonal projection onto $\mathfrak{h}_{\alpha}^{e l}$. In the following we treat only the case $F_{+}$. The calculations for $F_{-}$are completely analogous. We use that $\Phi$ maps $H_{0}^{a c}$ into the multiplication operator $\mathcal{M}$ induced by $\lambda$. Hence, we get the following:

$$
\begin{aligned}
& \left\|P_{1}^{\alpha} \widetilde{\gamma}\left(H_{0}\right) e^{-i t H_{0}} \Phi^{*} \mathcal{F} f\right\|=\left\|P_{1}^{\alpha} \Phi^{*} \Phi \widetilde{\gamma}\left(H_{0}\right) e^{-i t H_{0}} \Phi^{*} \mathcal{F} f\right\|= \\
& \quad=(2 \pi)^{-\frac{1}{2}}\left(\sum_{n \in \mathbb{N}_{0}}\left|\int_{\delta_{\alpha, n}} \mathrm{~d} \lambda g_{\alpha}(1, \lambda-n \omega) \widetilde{\gamma}(\lambda) \int_{\mathbb{R}_{+}} \mathrm{d} x e^{-i \lambda(x+t)} f(x)\right|^{2}\right)^{\frac{1}{2}}
\end{aligned}
$$

where $\operatorname{supp}(f) \subseteq \mathbb{R}_{+}, \widetilde{\gamma}(\lambda):=(\lambda-i)^{-1} \gamma(\lambda), \lambda \in \mathbb{R}$, and $\delta_{\alpha, n}:=\left[v_{\alpha}+n \omega_{0}, v_{\alpha}+n \omega+4\right]$. Notice that $\widetilde{\gamma}(\lambda) \in C_{0}^{\infty}(\mathbb{R} \backslash \Lambda)$. We find:

$$
\begin{aligned}
& \int_{\delta_{j, n}} \mathrm{~d} \lambda g_{\alpha}(1, \lambda-n \omega) \widetilde{\gamma}(\lambda) \int_{\mathbb{R}_{+}} \mathrm{d} x e^{-i \lambda(x+t)} f(x)= \\
& \int_{v_{\alpha}}^{v_{\alpha}+4} \mathrm{~d} \lambda g_{\alpha}(1, \lambda) \widetilde{\gamma}(\lambda+n \omega) \int_{\mathbb{R}_{+}} \mathrm{d} x e^{-i(\lambda+n \omega)(x+t)} f(x)
\end{aligned}
$$


which yields:

$$
\begin{aligned}
& \left\|P_{1}^{\alpha} \Phi^{*} \Phi \widetilde{\gamma}\left(H_{0}\right) e^{-i t H_{0}} \Phi^{*} \mathcal{F} f\right\|= \\
& (2 \pi)^{-\frac{1}{2}}\left(\sum_{n \in \mathbb{N}_{0}}\left|\int_{v_{\alpha}}^{v_{\alpha}+4} \mathrm{~d} \lambda g_{\alpha}(1, \lambda) \widetilde{\gamma}\left(\lambda+n \omega_{0}\right) \int_{\mathbb{R}_{+}} \mathrm{d} x e^{-i\left(\lambda+n \omega_{0}\right)(x+t)} f(x)\right|^{2}\right)^{\frac{1}{2}} .
\end{aligned}
$$

Since the support of $\gamma(\lambda)$ is compact, we see that the sum $\sum_{n \in \mathbb{N}_{0}}$ is finite. Changing the integrals, we get:

$$
\begin{aligned}
& \int_{\delta_{\alpha, n}} \mathrm{~d} \lambda g_{\alpha}(1, \lambda-n \omega) \widetilde{\gamma}(\lambda) \int_{\mathbb{R}_{+}} \mathrm{d} x e^{-i \lambda(x+t)} f(x)= \\
& \int_{\mathbb{R}_{+}} \mathrm{d} x f(x) e^{-i n \omega_{0}(x+t)} \int_{v_{\alpha}}^{v_{\alpha}+4} \mathrm{~d} \lambda g_{\alpha}(1, \lambda) \widetilde{\gamma}(\lambda+n \omega) e^{-i \lambda(x+t)}
\end{aligned}
$$

Integrating by parts $m$-times, we obtain:

$$
\begin{aligned}
& \int_{\delta_{\alpha, n}} \mathrm{~d} \lambda g_{\alpha}(1, \lambda-n \omega) \widetilde{\gamma}(\lambda) \int_{\mathbb{R}_{+}} \mathrm{d} x e^{-i \lambda(x+t)} f(x)= \\
& \quad(-i)^{m} \int_{\mathbb{R}_{+}} \mathrm{d} x f(x) \frac{e^{-i n \omega(x+t)}}{(x+t)^{m}} \int_{v_{\alpha}}^{v_{\alpha}+4} \mathrm{~d} \lambda e^{-i \lambda(x+t)} \frac{d^{m}}{d \lambda^{m}}\left(g_{\alpha}(1, \lambda) \widetilde{\gamma}(\lambda+n \omega)\right)
\end{aligned}
$$

Hence,

$$
\left|\int_{\delta_{\alpha, n}} \mathrm{~d} \lambda g_{\alpha}(1, \lambda-n \omega) \widetilde{\gamma}(\lambda) \int_{\mathbb{R}_{+}} \mathrm{d} x e^{-i \lambda(x+t)} f(x)\right|^{2} \leqslant C_{n}^{2}\left(\int_{\mathbb{R}_{+}} \mathrm{d} x|f(x)| \frac{1}{(x+t)^{m}}\right)^{2}
$$

which yields:

$$
\left|\int_{\delta_{\alpha, n}} \mathrm{~d} \lambda g_{\alpha}(1, \lambda-n \omega) \widetilde{\gamma}(\lambda) \int_{\mathbb{R}_{+}} \mathrm{d} x e^{-i \lambda(x+t)} f(x)\right|^{2} \leqslant C_{n}^{2} \frac{1}{t^{(2 m-1)}}\|f\|^{2}
$$

for $m \in \mathbb{N}$ where:

$$
C_{n}:=\int_{v_{\alpha}}^{v_{\alpha}+4} \mathrm{~d} \lambda \mid \frac{d^{m}}{d \lambda^{m}}\left(g_{\alpha}(1, \lambda) \widetilde{\gamma}(\lambda+n \omega) \mid\right) .
$$

Notice that $C_{n}=0$ for sufficiently large $n \in \mathbb{N}$. Therefore,

$$
\left\|P_{1}^{\alpha} \widetilde{\gamma}\left(H_{0}\right) e^{-i t H_{0}} \Phi^{*} \mathcal{F} f\right\| \leqslant\left(\sum_{n \in \mathbb{N}_{0}} C_{n}^{2}\right)^{1 / 2} \frac{1}{t^{m-1 / 2}}\|f\|, \quad f \in L^{2}\left(\mathbb{R}_{+}, d x\right),
$$

which shows that $\left\|P_{1}^{\alpha} \widetilde{\gamma}\left(H_{0}\right) e^{-i t H_{0}} F_{+}\right\| \in L^{1}\left(\mathbb{R}_{+}, d t\right)$ for $m \geqslant 2$.

\section{Landauer-Büttiker formula and applications}

\subsection{Landauer-Büttiker formula}

The abstract Landauer-Büttiker formula can be used to calculate currents through devices. Usually one considers a pair $\mathcal{S}=\left\{K, K_{0}\right\}$ be of self-adjoint operators where the unperturbed Hamiltonian $K_{0}$ describes a totally decoupled system, that means, the inner system is closed and the leads are decoupled from it, while the perturbed Hamiltonian $K$ describes the system where the leads are coupled to the inner system. An important component is system $\mathcal{S}=\left\{K, K_{0}\right\}$, which represents a complete scattering or even a trace class scattering system.

In [1], an abstract Landauer-Büttiker formula was derived in the framework of a trace class scattering theory for semi-bounded self-adjoint operators which allows one to reproduce the results of [18] and [7] rigorously. In [13], the results of [1] were generalized to non-semibounded operators. Following [1], we consider a trace class scattering system $\mathcal{S}=\left\{K, K_{0}\right\}$. We 
recall that $\mathcal{S}=\left\{K, K_{0}\right\}$ is called a trace class scattering system if the resolvent difference of $K$ and $K_{0}$ belongs to the trace class. If $\mathcal{S}=\left\{K, K_{0}\right\}$ is a trace class scattering system, then the wave operators $W_{ \pm}\left(K, K_{0}\right)$ exist and are complete. The scattering operator is defined by $S\left(K, K_{0}\right):=W_{+}\left(K, K_{0}\right)^{*} W_{-}\left(K, K_{0}\right)$. The main components, besides the trace class scattering system $\mathcal{S}=\left\{K, K_{0}\right\}$, are the density and the charge operators $\rho$ and $Q$, respectively.

The density operator $\rho$ is a non-negative bounded self-adjoint operator commuting with $K_{0}$. The charge $Q$ is a bounded self-adjoint operator commuting also with $K_{0}$. If $K$ has no singular continuous spectrum, then the current related to the density operator $\rho$ and the charge $Q$ is defined as follows:

$$
J_{\rho, Q}^{\mathcal{S}}=-i \operatorname{tr}\left(W_{-}\left(K, K_{0}\right) \rho W_{-}\left(K, K_{0}\right)^{*}[K, Q]\right),
$$

where $[K, Q]$ is the commutator of $K$ and $Q$. In fact, the commutator $[K, Q]$ might be not defined. In this case, the regularized definition:

$$
J_{\rho, Q}^{\mathcal{S}}=-i \operatorname{tr}\left(W_{-}\left(K, K_{0}\right)\left(I+K_{0}^{2}\right) \rho W_{-}\left(K, K_{0}\right)^{*} \frac{1}{K-i}[K, Q] \frac{1}{K+i}\right),
$$

is used, where it is assumed that $\left(I+K_{0}^{2}\right) \rho$ is a bounded operator. Since the condition $(H-i)^{-1}[H, Q](H+i)^{-1} \in \mathfrak{L}_{1}(\mathfrak{H})$ is satisfied, definition (3.2) makes sense. By $\mathfrak{L}_{1}(\mathfrak{H})$ is the ideal of trace class operators is denoted.

Let $K_{0}$ be self-adjoint operator on the separable Hilbert space $\mathfrak{K}$. We call $\rho$ be a density operator for $K_{0}$ if $\rho$ is a bounded non-negative self-adjoint operator commuting with $K_{0}$. Since $\rho$ commutes with $K_{0}$, one sees that $\rho$ leaves invariant the subspace $\mathfrak{K}^{a c}\left(K_{0}\right)$. We then set

$$
\rho_{a c}:=\rho \uparrow \mathfrak{K}^{a c}\left(K_{0}\right) \text {. }
$$

call $\rho_{a c}$ the $a c$-density part of $\rho$.

A bounded self-adjoint operator, $Q$, commuting with $K_{0}$, is called a charge. If $Q$ is the charge, then:

$$
Q_{a c}:=Q \uparrow \mathfrak{K}^{a c}\left(K_{0}\right),
$$

is called its $a c$-charge component.

Let $\Pi\left(K_{0}^{a c}\right)=\left\{L^{2}(\mathbb{R}, d \lambda, \mathfrak{k}(\lambda)), \mathcal{M}, \Phi\right\}$ be a spectral representation of $K_{0}^{a c}$. If $\rho$ is a density operator, then there is a measurable family $\left\{\rho_{a c}(\lambda)\right\}_{\lambda \in \mathbb{R}}$ of bounded self-adjoint operators such that the multiplication operator:

$$
\left(\mathcal{M}_{\rho_{a c}} \widehat{f}\right)(\lambda):=\rho_{a c}(\lambda) \widehat{f}(\lambda), \quad \widehat{f} \in \operatorname{dom}\left(M_{\rho_{a c}}\right):=L^{2}(\mathbb{R}, d \lambda, \mathfrak{k}(\lambda)),
$$

is unitarily equivalent to the $a c$-part $\rho_{a c}$, that is, $\mathcal{M}_{\rho_{a c}}=\Phi \rho_{a c} \Phi^{*}$. In particular, this yields that: $\operatorname{ess-sup}_{\lambda \in \mathbb{R}}\left\|\rho_{a c}(\lambda)\right\|_{\mathcal{B}(\mathfrak{k}(\lambda)}=\left\|\rho_{a c}\right\|_{\mathcal{B}\left(\mathfrak{K}^{a c}\left(K_{0}\right)\right)}$. In the following, we call $\left\{\rho_{a c}(\lambda)\right\}_{\lambda \in \mathbb{R}}$ the density matrix of $\rho_{a c}$.

Similarly, one obtains that if $Q$ is a charge, then there is a measurable family $\left\{Q_{a c}(\lambda)\right\}_{\lambda \in \mathbb{R}}$ of bounded self-adjoint operators, such that the multiplication operator:

$$
\begin{aligned}
\left(\mathcal{M}_{Q_{a c}} \widehat{f}\right)(\lambda) & :=Q_{a c}(\lambda) \widehat{f}(\lambda), \\
\widehat{f} \in \operatorname{dom}\left(Q_{a c}\right) & :=\left\{f \in L^{2}(\mathbb{R}, d \lambda, \mathfrak{k}(\lambda)): Q_{a c}(\lambda) \widehat{f}(\lambda) \in L^{2}(\mathbb{R}, d \lambda, \mathfrak{k}(\lambda))\right\},
\end{aligned}
$$

is unitarily equivalent to $Q_{a c}$, i.e. $\mathcal{M}_{Q_{a c}}=\Phi Q_{a c} \Phi^{*}$. In particular, one has:

$$
\operatorname{ess} \sup _{\lambda \in \mathbb{R}}\left\|Q_{a c}(\lambda)\right\|_{\mathcal{B}(\mathfrak{k}(\lambda))}=\left\|Q_{a c}\right\|_{\mathcal{B}\left(\mathfrak{K} \mathfrak{R}^{a c}\left(K_{0}\right)\right)} \text {. }
$$

If $Q$ is a charge, then the family $\left\{Q_{a c}(\lambda)\right\}_{\lambda \in \mathbb{R}}$ is called the charge matrix of the ac-component of $Q$.

Let $\mathcal{S}=\left\{K, K_{0}\right\}$ be a trace scattering system. By $\{S(\lambda)\}_{\lambda \in \mathbb{R}}$, we denote the scattering matrix, which corresponds to the scattering operator $S\left(K, K_{0}\right)$ with respect to the spectral representation $\Pi\left(K_{0}^{a c}\right)$. The operator $T:=S\left(K, K_{0}\right)-P^{a c}\left(K_{0}\right)$ is called the transmission operator. 
By $\{T(\lambda)\}_{\lambda \in \mathbb{R}}$, we denote the transmission matrix which is related to the transmission operator. Scattering and transmission matrices are related by $S(\lambda)=T_{\mathfrak{k}(\lambda)}+T(\lambda)$ for a.e. $\lambda \in \mathbb{R}$. Notice that $T(\lambda)$ belongs for to the trace class a.e. $\lambda \in \mathbb{R}$.

Theorem 3.1 ( [13, Corollary 2.14]). Let $S:=\left\{K, K_{0}\right\}$ be a trace class scattering system and let $\{S(\lambda)\}_{\lambda \in \mathbb{R}}$ be the scattering matrix of $\mathcal{S}$ with respect to the spectral representation $\Pi\left(K_{0}^{a c}\right)$. Furthermore, let $\rho$ and $Q$ be density and charge operators and let $\left\{\rho_{a c}(\lambda)\right\}_{\lambda \in \mathbb{R}}$ and $\left\{Q_{a c}(\lambda)\right\}_{\lambda \in \mathbb{R}}$ be the density and charge matrices of the ac-components $\rho_{a c}$ and charge $Q_{a c}$ with respect to $\Pi\left(K_{0}^{a c}\right)$, respectively. If $\left(I+K_{0}^{2}\right) \rho$ is bounded, then the current $J_{\rho, Q}^{\mathcal{S}}$ defined by (3.2) admits the representation:

$$
J_{\rho, Q}^{\mathcal{S}}=\frac{1}{2 \pi} \int_{\mathbb{R}} \operatorname{tr}\left(\rho_{a c}(\lambda)\left(Q_{a c}(\lambda)-S^{*}(\lambda) Q_{a c}(\lambda) S(\lambda)\right)\right) \mathrm{d} \lambda,
$$

where the integrand on the right side and the current $J_{\rho, Q}^{\mathcal{S}}$ satisfy the estimate:

$$
\begin{array}{r}
\left|\operatorname{tr}\left(\rho_{a c}(\lambda)\left(Q_{a c}(\lambda)-S^{*}(\lambda) Q_{a c}(\lambda) S(\lambda)\right)\right)\right| \leqslant \\
\quad 4\|\rho(\lambda)\|_{\mathfrak{L}(\mathfrak{k}(\lambda))}\|T(\lambda)\|_{\mathfrak{L}_{1}(\mathfrak{k}(\lambda))}\|Q(\lambda)\|_{\mathfrak{L}(\mathfrak{k}(\lambda))},
\end{array}
$$

for a.e. $\lambda \in \mathbb{R}$ and

$$
\left|J_{\rho, Q}^{\mathcal{S}}\right| \leqslant C_{0}\left\|(H+i)^{-1}-\left(H_{0}+i\right)^{-1}\right\|_{\mathfrak{L}_{1}(\mathfrak{K})},
$$

where $C_{0}:=\frac{2}{\pi}\left\|\left(1+H_{0}^{2}\right) \rho\right\|_{\mathfrak{L}(\mathfrak{K})}$.

In applications, not every charge $Q$ is a bounded operator. We say the self-adjoint operator $Q$ commuting with $K_{0}$ is a $p$-tempered charge if $Q\left(H_{0}-i\right)^{-p}$ is a bounded operator for $p \in \mathbb{N}_{0}$. As above, we can introduce $Q_{a c}:=Q \uparrow \operatorname{dom}(Q) \cap \mathfrak{K}^{a c}\left(K_{0}\right)$. It follows that $Q E_{K_{0}}(\Delta)$ is a bounded operator for any bounded Borel set $\Delta$. This yields that the corresponding charge matrix, $\left\{Q_{a c}(\lambda)\right\}_{\lambda \in \mathbb{R}}$, is a measurable family of bounded self-adjoint operators such that:

$$
\operatorname{ess} \sup _{\lambda \in \mathbb{R}}\left(1+\lambda^{2}\right)^{p / 2}\left\|Q_{a c}(\lambda)\right\|_{\mathfrak{L}(\mathfrak{k}(\lambda))}<\infty .
$$

To generalize the current $J_{\rho, Q}^{\mathcal{S}}$ to tempered charges $Q$, one uses the fact that $Q(\Delta):=Q E_{K_{0}}(\Delta)$ is a charge for any bounded Borel set $\Delta$. Hence, the current $J_{\rho, Q(\Delta)}^{\mathcal{S}}$ is well-defined by (3.2) for any bounded Borel set $\Delta$. Using Theorem 3.1 one gets that for $p$-tempered charges, the limit

$$
J_{\rho, Q}^{\mathcal{S}}:=\lim _{\Delta \rightarrow \mathbb{R}} J_{\rho, Q(\Delta)}^{\mathcal{S}}
$$

exists, provided $\left(H_{0}-i\right)^{p+2} \rho$ is a bounded operator. This gives rise to the following corollary:

Corollary 3.2. Let the assumptions of the Theorem 3.1 be satisfied. If for some $p \in \mathbb{N}_{0}$ the operator $\left(H_{0}-i\right)^{p+2} \rho$ is bounded and $Q$ is a p-tempered charge for $K_{0}$, then the current defined by (3.7) admits the representation (3.4), where the right hand side of (3.4) satisfies the estimate (3.5). Moreover, the current $J_{\rho, Q}^{\mathcal{S}}$ can be estimated in the following manner:

$$
\left|J_{\rho, Q}^{\mathcal{S}}\right| \leqslant C_{p}\left\|(H+i)^{-1}-\left(H_{0}+i\right)^{-1}\right\|_{\mathfrak{L}_{1}(\mathfrak{K})},
$$

where $C_{p}:=\frac{2}{\pi}\left\|\left(1+H_{0}^{2}\right)^{p+2 / 2} \rho\right\|_{\mathfrak{L}(\mathfrak{K})}\left\|Q\left(I+H_{0}^{2}\right)^{-p / 2}\right\|_{\mathfrak{L}(\mathfrak{K})}$.

At first glance, the formula (3.4) is not very similar to the original Landauer-Büttiker formula of $[7,18]$. To make the formula more convenient, we recall that a standard application example for the Landauer-Büttiker formula is the so-called black-box model, cf. [1]. In this case, the Hilbert space $\mathfrak{K}$ is given by:

$$
\mathfrak{K}=\mathfrak{K}_{S} \oplus \bigoplus_{j=1}^{N} \mathfrak{K}_{j}, \quad 2 \leqslant N<\infty .
$$


and $K_{0}$ by:

$$
K_{0}=K_{S} \oplus \bigoplus_{j=1}^{N} K_{j}, \quad 2 \leqslant N<\infty .
$$

The Hilbert space $\mathfrak{K}_{S}$ is called the sample or dot and $K_{S}$ is the sample or dot Hamiltonian. The Hilbert spaces $\mathfrak{K}_{j}$ are called reservoirs or leads and $K_{j}$ are the reservoir or lead Hamiltonians. For simplicity, we assume that the reservoir Hamiltonians $K_{j}$ are absolutely continuous and the sample Hamiltonian $K_{S}$ has a point spectrum. A typical choice for the density operator is:

$$
\rho=f_{S}\left(K_{S}\right) \oplus \bigoplus_{j=1}^{N} f_{j}\left(K_{j}\right),
$$

where $f_{S}(\cdot)$ and $f_{j}(\cdot)$ are non-negative bounded Borel functions, and for the charge:

$$
Q=g_{S}\left(H_{s}\right) \oplus \bigoplus_{j=1}^{N} g_{j}\left(H_{j}\right)
$$

where $g_{S}(\cdot)$ and $g_{j}(\cdot)$ a bounded Borel functions. Making this choice the Landauer-Büttiker formula (3.4) takes the form:

$$
J_{\rho, Q}^{\mathcal{S}}=\frac{1}{2 \pi} \sum_{j, k=1}^{N} \int_{\mathbb{R}}\left(f_{j}(\lambda)-f_{k}(\lambda)\right) g_{j}(\lambda) \sigma_{j k}(\lambda) d \lambda,
$$

where

$$
\sigma_{j k}(\lambda):=\operatorname{tr}\left(T_{j k}(\lambda)^{*} T_{j k}(\lambda)\right), \quad j, k=1, \ldots, N, \quad \lambda \in \mathbb{R},
$$

are called the total transmission probability from reservoir $k$ to reservoir $j$, cf. [1]. We call it the cross-section of the scattering process going from channel $k$ to channel $j$ at energy $\lambda \in \mathbb{R}$. $\left\{T_{j k}(\lambda)\right\}_{\lambda \in \mathbb{R}}$ is called the transmission matrix from channel $k$ to channel $j$ at energy $\lambda \in \mathbb{R}$ with respect to the spectral representation $\Pi\left(K_{0}^{a c}\right)$. We note that $\left\{T_{j k}(\lambda)\right\}_{\lambda \in \mathbb{R}}$ corresponds to the transmission operator:

$$
T_{j k}:=P_{j} T\left(K, K_{0}\right) P_{k}, \quad T\left(K, K_{0}\right):=S\left(K, K_{0}\right)-P^{a c}\left(K_{0}\right),
$$

acting from the reservoir $k$ to reservoir $j$ where $T\left(K, K_{0}\right)$ is called the transmission operator. Let $\{T(\lambda)\}_{\lambda \in \mathbb{R}}$ be the transmission matrix. Following [1], the current $J_{\rho, Q}^{\mathcal{S}}$ given by (3.13) is directed from the reservoirs into the sample.

The quantity $\|T(\lambda)\|_{\mathfrak{L}_{2}}=\operatorname{tr}(T(\lambda) * T(\lambda))$ is well-defined and is called the cross-section of the scattering system $\mathcal{S}$ at energy $\lambda \in \mathbb{R}$. Notice that:

$$
\sigma(\lambda)=\|T(\lambda)\|_{\mathfrak{L}_{2}}=\operatorname{tr}\left(T(\lambda)^{*} T(\lambda)\right)=\sum_{j, k=1}^{N} \sigma_{j k}(\lambda) . \quad \lambda \in \mathbb{R},
$$

We point out that the channel cross-sections $\sigma_{j k}(\lambda)$ admit the property:

$$
\sum_{j=1}^{N} \sigma_{j k}(\lambda)=\sum_{j=1}^{N} \sigma_{k j}(\lambda), \quad \lambda \in \mathbb{R}
$$

which is a consequence of the unitarity of the scattering matrix. Moreover, if there is a conjugation $J$, such that $K J=J K$ and $K_{0} J=J K_{0}$ holds, that is, if the scattering system $\mathcal{S}$ is time reversible symmetric, then we have even more, namely, it holds that:

$$
\sigma_{j k}(\lambda)=\sigma_{k j}(\lambda), \quad \lambda \in \mathbb{R} .
$$

Usually, the Landauer-Büttiker formula (3.13) is used to calculated the electron current entering the reservoir $j$ from the sample. In this case one has to choose $Q:=Q_{j}^{e l}:=-\mathfrak{e} P_{j}$ where 
$P_{j}$ is the orthogonal projection form $\mathfrak{K}$ onto $\mathfrak{K}_{j}$ and $\mathfrak{e}>0$ is the magnitude of the elementary charge. This is equivalent to choosing $g_{j}(\lambda)=-\mathfrak{e}$ and $g_{k}(\lambda)=0$ for $k \neq j, \lambda \in \mathbb{R}$. In doing so, we get the Landauer-Büttiker formula simplifying to:

$$
J_{\rho, Q_{j}^{e l}}^{\mathcal{S}}=-\frac{\mathfrak{e}}{2 \pi} \sum_{k=1}^{N} \int_{\mathbb{R}}\left(f_{j}(\lambda)-f_{k}(\lambda)\right) \sigma_{j k}(\lambda) d \lambda .
$$

To restore the original Landauer-Büttiker formula, one sets:

$$
f_{j}(\lambda)=f\left(\lambda-\mu_{j}\right), \quad \lambda \in \mathbb{R},
$$

where $\mu_{j}$ is the chemical potential of the reservoir $\mathfrak{K}_{j}$ and $f(\cdot)$ is a bounded non-negative Borel function called the distribution function. This gives rise to the following formula:

$$
J_{\rho, Q_{j}^{e l}}^{\mathcal{S}}=-\frac{\mathfrak{e}}{2 \pi} \sum_{k=1}^{N} \int_{\mathbb{R}}\left(f\left(\lambda-\mu_{j}\right)-f\left(\lambda-\mu_{k}\right)\right) \sigma_{j k}(\lambda) d \lambda .
$$

In particular, if we choose one:

$$
f(\lambda):=f_{F D}(\lambda):=\frac{1}{1+e^{\beta \lambda}}, \quad \beta>0, \quad \lambda \in \mathbb{R},
$$

where $f_{F D}(\cdot)$ is the Fermi-Dirac distribution function, and inserting (3.21) into (3.20) we obtain:

$$
J_{\rho, Q_{j}^{e l}}^{\mathcal{S}}=-\frac{\mathfrak{e}}{2 \pi} \sum_{k=1}^{N} \int_{\mathbb{R}}\left(f_{F D}\left(\lambda-\mu_{j}\right)-f_{F D}\left(\lambda-\mu_{k}\right)\right) \sigma_{j k}(\lambda) d \lambda .
$$

If we have only two reservoirs, then they are usually denoted by $l$ (left) and $r$ (right). Let $j=l$ and $k=r$. Then,

$$
J_{\rho, Q_{l}^{e l}}^{\mathcal{S}}=-\frac{\mathfrak{e}}{2 \pi} \int_{\mathbb{R}}\left(f_{F D}\left(\lambda-\mu_{l}\right)-f_{F D}\left(\lambda-\mu_{r}\right)\right) \sigma_{l r}(\lambda) d \lambda .
$$

One easily checks that $J_{\rho, Q_{l}}^{\mathcal{S}} \leqslant 0$ if $\mu_{l} \geqslant \mu_{r}$. That means, the current is leaving the left reservoir and is entering the right one which is in accordance with physical expectations.

Example 3.3. Notice that $s_{c}:=\left\{h^{e l}, h_{0}^{e l}\right\}$ is a $\mathfrak{L}_{1}$ scattering system. The Hamiltonian $h^{e l}$ takes into account the effect of coupling of reservoirs or leads $\mathfrak{h}_{l}:=l^{2}(\mathbb{N})$ and $\mathfrak{h}_{r}:=l^{2}(\mathbb{N})$ to the sample $\mathfrak{h}_{S}=\mathbb{C}^{2}$ which is also called the quantum dot. The Hamiltonians for the leads are given by: $h_{\alpha}^{e l}=-\Delta^{D}+v_{\alpha}, \alpha=l, r$. The sample or quantum dot Hamiltonian is given by $h_{S}^{e l}$. The wave operators are given by:

$$
w_{ \pm}\left(h^{e l}, h_{0}^{e l}\right):=s-\lim _{t \rightarrow \infty} e^{i t h^{e l}} e^{-i t h_{0}^{e l}} P^{a c}\left(h_{0}^{e l}\right) .
$$

The scattering operator is given by $s_{c}:=w_{+}\left(h^{e l}, h_{0}^{e l}\right)^{*} w_{-}\left(h^{e l}, h_{0}^{e l}\right)$. Let $\Pi\left(h_{0}^{e l, a c}\right)$ be the spectral representation of $h_{0}^{e l, a c}$ introduced in Section 2.6. If $\rho^{e l}$ and $q^{e l}$ are density and charge operators for $h_{0}^{e l}$, then the Landauer-Büttiker formula takes the following form:

$$
J_{\rho^{e l}, q^{e l}}^{s_{c}}=\frac{1}{2 \pi} \int_{\mathbb{R}} \operatorname{tr}\left(\rho_{a c}^{e l}(\lambda)\left(q_{a c}^{e l}-s_{c}(\lambda)^{*} q_{a c}^{e l}(\lambda) s_{c}(\lambda)\right)\right),
$$

where $\left\{s_{c}(\lambda)\right\}_{\lambda \in \mathbb{R}},\left\{q^{e l}(\lambda)\right\}_{\lambda \in \mathbb{R}}$ and $\left\{\rho^{e l}(\lambda)\right\}_{\lambda \in \mathbb{R}}$ are the scattering, charge and density matrices with respect to $\Pi\left(h_{0}^{e l, a c}\right)$, respectively. The condition that $\left(\left(h_{0}^{e l}\right)^{2}+I_{\mathfrak{h}^{e l}}\right) \rho^{e l}$ is a bounded operator is superfluous because $h_{0}^{e l}$ is a bounded operator. For the same reason we have that every $p$-tempered charge $q^{e l}$ is in fact a charge, that means, $q^{e l}$ is a bounded self-adjoint operator.

The scattering system $s_{c}$ is a black-box model with reservoirs $\mathfrak{h}_{l}^{\text {el }}$ and $\mathfrak{h}_{r}^{\text {el }}$. Choosing

$$
\rho^{e l}=f_{l}\left(h^{e l}\right) \oplus f_{S}\left(h_{S}^{e l}\right) \oplus f_{r}\left(h_{r}^{e l}\right),
$$


where $f_{\alpha}(\cdot), \alpha=l, r$, are bounded Borel functions, and

$$
q^{e l}=g_{l}\left(h_{l}^{e l}\right) \oplus g_{S}\left(h_{S}^{e l}\right) \oplus g_{r}\left(h_{r}^{e l}\right),
$$

where $g_{\alpha}(\cdot), \alpha \in\{l, r\}$, are locally bounded Borel functions, then from (3.13) it follows that:

$$
J_{\rho^{e l}, q^{e l}}^{s_{c}}=\frac{1}{2 \pi} \sum_{\substack{\alpha, \varkappa \in\{l, r\} \\ \alpha \neq \varkappa}} \int_{\mathbb{R}}\left(f_{\alpha}(\lambda)-f_{\varkappa}(\lambda)\right) g_{\alpha}(\lambda) \sigma_{c}(\lambda) d \lambda,
$$

where $\left\{\sigma_{c}(\lambda)\right\}_{\lambda \in \mathbb{R}}$ is the channel cross-section from left to right and vice versa. Indeed, let $\left\{t_{c}(\lambda)\right\}_{\lambda \in \mathbb{R}}$ be the transition matrix which corresponds to the transition operator $t_{c}:=s_{c}-I_{\mathfrak{h}^{e l}}$. Clearly, one has $t_{c}(\lambda)=I_{\mathfrak{h}(\lambda)}-s_{c}(\lambda), \lambda \in \mathbb{R}$. Let $\left\{p_{\alpha}^{e l}(\lambda)\right\}_{\lambda \in \mathbb{R}}$ be the matrix which corresponds to the orthogonal projection $p_{\alpha}^{e l}$ from $\mathfrak{h}^{e l}$ onto $\mathfrak{h}_{\alpha}^{e l}$. Further, let $t_{r l}^{c}(\lambda):=p_{r}^{e l}(\lambda) t_{c}(\lambda) p_{l}^{e l}$ and $t_{l r}^{c}:=p_{l}^{e l}(\lambda) t_{c}(\lambda) p_{r}^{e l}$. Notice that both quantities are in fact scalar functions. Accordingly, the channel cross-sections $\sigma_{l r}^{c}(\lambda)$ and $\sigma_{r l}^{c}(\lambda)$ at energy $\lambda \in \mathbb{R}$ are given by $\sigma_{c}(\lambda):=\sigma_{l r}^{c}(\lambda)=$ $\left|t_{l r}^{c}(\lambda)\right|^{2}=\left|t_{r l}^{c}(\lambda)\right|^{2}=\sigma_{r l}^{c}(\lambda), \lambda \in \mathbb{R}$.

In particular, if $g_{l}(\lambda)=1$ and $g_{r}=0$, then:

$$
J_{\rho^{e l}, q_{l}^{e l}}^{s_{c}}=\frac{1}{2 \pi} \int_{\mathbb{R}}\left(f_{l}(\lambda)-f_{r}(\lambda)\right) \sigma_{c}(\lambda) d \lambda,
$$

and $q_{l}^{e l}:=p_{l}^{e l}$. Following [1], $J_{\rho^{e l}, q_{l}^{e l}}^{s_{c}}$ denotes the current entering the quantum dot from the left lead.

\subsection{Application to the $J C L$-model}

Let $\mathcal{S}=\left\{H, H_{0}\right\}$ now be the $J C L$-model. Furthermore, let $\rho$ and $Q$ be the density operator and a charge for $H_{0}$, respectively. Under these assumptions, the current $J_{\rho, Q}^{\mathcal{S}}$ is defined by:

$$
J_{\rho, Q}^{\mathcal{S}}:=-i \operatorname{tr}\left(W_{-}\left(H, H_{0}\right)\left(I+H_{0}^{2}\right) \rho W_{-}\left(H, H_{0}\right)^{*} \frac{1}{H-i}[H, Q] \frac{1}{H+i}\right),
$$

and admits representation (3.4). If $Q$ is a $p$-tempered charge and $\left(H_{0}-i\right)^{p+2} \rho$ is a bounded operator, then the current $J_{\rho, Q}^{\mathcal{S}}$ is defined in accordance with (3.7) and the Landauer-Büttiker formula (3.4) is also valid.

We introduce the intermediate scattering system $\mathcal{S}_{c}:=\left\{H, H_{c}\right\}$, where:

$$
H_{c}:=h^{e l} \otimes I_{\mathfrak{h}^{p h}}+I_{\mathfrak{h}^{e l}} \otimes h^{p h}=H_{0}+V_{e l} .
$$

The Hamiltonian $H_{c}$ describes the coupling of the leads to the quantum dot, but under the assumption that the photon interaction is not switched on.

Accordingly, $\mathcal{S}_{p h}:=\left\{H, H_{c}\right\}$ and $\mathcal{S}_{c}:=\left\{H_{c}, H_{0}\right\}$ are $\mathfrak{L}_{1}$-scattering systems. The corresponding scattering operators are denoted by $S_{p h}$ and $S_{c}$, respectively. Let $\Pi\left(H_{c}^{a c}\right)=$ $\left\{L^{2}\left(\mathbb{R}, d \lambda, \mathfrak{h}_{c}(\lambda)\right), \mathcal{M}, \Phi_{c}\right\}$ of $H_{c}^{a c}$ be a spectral representation of $H_{c}$. The scattering matrix of the scattering system $\left\{H, H_{c}\right\}$ with respect to $\Pi\left(H_{c}^{a c}\right)$ is denoted by $\left\{S_{p h}(\lambda)\right\}_{\lambda \in \mathbb{R}}$. The scattering matrix of the scattering system $\left\{H_{c}, H_{0}\right\}$ with respect to $\Pi\left(H_{0}^{a c}\right)=\left\{L^{2}\left(\mathbb{R}, d \lambda, \mathfrak{h}_{0}(\lambda)\right), \mathcal{M}, \Phi_{0}\right\}$ is denoted by $\left\{S_{c}(\lambda)\right\}_{\lambda \in \mathbb{R}}$.

Since $\mathcal{S}_{c}$ is a $\mathfrak{L}_{1}$-scattering system, the wave operators $W_{ \pm}\left(H_{c}, H_{0}\right)$ exist and are complete and since $\Phi_{c} W_{ \pm}\left(H_{c}, H_{0}\right) \Phi_{0}^{*}$ commutes with $\mathcal{M}$, there are measurable families $\left\{W_{ \pm}(\lambda)\right\}_{\lambda \in \mathbb{R}}$ of isometries acting from $\mathfrak{h}_{0}(\lambda)$ onto $\mathfrak{h}_{c}(\lambda)$ for a.e. $\lambda \in \mathbb{R}$ such that:

$$
\left(\Phi_{c} W_{ \pm}\left(H_{c}, H_{0}\right) \Phi_{0}^{*} \widehat{f}\right)(\lambda)=W_{ \pm}(\lambda) \widehat{f}(\lambda), \quad \lambda \in \mathbb{R}, \quad \widehat{f} \in L^{2}\left(\mathbb{R}, d \lambda, \mathfrak{h}_{0}(\lambda)\right) .
$$

The families $\left\{W_{ \pm}(\lambda)\right\}_{\lambda \in \mathbb{R}}$ are called wave matrices.

Straightforward computation shows that $\widehat{S}_{p h}:=W_{+}\left(H_{c}, H_{0}\right)^{*} S_{p h} W_{+}\left(H_{c}, H_{0}\right)$ commutes with $H_{0}$. Hence, with respect to the spectral representation $\Pi\left(H_{0}^{a c}\right)$, the operator $\widehat{S}_{p h}$ is unitarily 
equivalent to a multiplication induced by a measurable family $\left\{\widehat{S}_{p h}(\lambda)\right\}_{\lambda \in \mathbb{R}}$ of unitary operators in $\mathfrak{h}_{0}(\lambda)$. Straightforward computation shows that:

$$
\widehat{S}_{p h}(\lambda)=W_{+}(\lambda)^{*} S_{p h}(\lambda) W_{+}(\lambda),
$$

for a.e. $\lambda \in \mathbb{R}$. Roughly speaking, $\left\{\widehat{S}_{p h}(\lambda)\right\}_{\lambda \in \mathbb{R}}$ is the scattering matrix of $S_{p h}$ with respect to the spectral representation $\Pi\left(H_{0}^{a c}\right)$.

Furthermore, let

$$
\rho^{c}:=W_{-}\left(H_{c}, H_{0}\right) \rho W_{-}\left(H_{c}, H_{0}\right)^{*}
$$

and

$$
Q^{c}:=W_{+}\left(H_{c}, H_{0}\right) Q W_{+}\left(H_{c}, H_{0}\right)^{*} .
$$

The operators $\rho^{c}$ and $Q^{c}$ are the density and tempered charge operators for the scattering system $S_{p h}$. Indeed, one easily verifies that $\rho^{c}$ and $Q^{c}$ are commute with $H_{c}$. Moreover, $\rho^{c}$ is non-negative. Furthermore, if $Q$ is a charge, then $Q^{c}$ is also a charge. This gives rise to the introduction of currents $J_{\rho, Q}^{c}:=J_{\rho, Q}^{S_{c}}$,

$$
J_{\rho, Q}^{c}:=-i \operatorname{tr}\left(W_{-}\left(H_{c}, H_{0}\right) \rho W_{-}\left(H_{c}, H_{0}\right)^{*} \frac{1}{H_{c}-i}\left[H_{c}, Q\right] \frac{1}{H_{c}+i}\right),
$$

and $J_{\rho, Q}^{p h}:=J_{\rho^{c}, Q^{c}}^{\mathcal{S}_{p h}}$

$$
J_{\rho, Q}^{p h}:=-i \operatorname{tr}\left(W_{-}\left(H, H_{c}\right) \rho^{c} W_{-}\left(H, H_{c}\right)^{*} \frac{1}{H-i}\left[H, Q^{c}\right] \frac{1}{H+i}\right),
$$

which are well defined. If $Q$ is $p$-tempered charge and $\left(H_{0}-i\right)^{p+2} \rho$ is a bounded operator, then one easily checks that $Q^{c}$ is a $p$-tempered charge and $\left(H_{c}-i\right)^{p+2} \rho^{c}$ is a bounded operator. Hence the definition of the currents $J_{\rho^{c}, Q^{c}}^{S_{c}}$ can be extended to this case and the Landauer-Büttiker formula (3.4) holds.

Finally, we note that the corresponding matrices $\left\{\rho_{a c}^{c}(\lambda)\right\}_{\lambda \in \mathbb{R}}$ and $\left\{Q_{a c}^{c}(\lambda)\right\}_{\lambda \in \mathbb{R}}$ are related to the matrices $\left\{\rho_{a c}(\lambda)\right\}_{\lambda \in \mathbb{R}}$ and $\left\{Q_{a c}(\lambda)\right\}_{\lambda \in \mathbb{R}}$ by

$$
\rho_{a c}^{c}(\lambda)=W_{-}(\lambda) \rho_{a c}(\lambda) W_{-}(\lambda)^{*} \quad \text { and } \quad Q_{a c}^{c}(\lambda)=W_{+}(\lambda) Q_{a c}(\lambda) W_{+}(\lambda)^{*}
$$

for a.e. $\lambda \in \mathbb{R}$.

Proposition 3.4 (Current decomposition). Let $\mathcal{S}=\left\{H, H_{0}\right\}$ be the JCL-model. Furthermore, let $\rho$ and $Q$ be the density operator and a p-tempered charge, $p \in \mathbb{N}_{0}$, for $H_{0}$, respectively. If $\left(H_{0}-i\right)^{p+2} \rho$ is a bounded operator, then the decomposition,

$$
J_{\rho, Q}^{\mathcal{S}}=J_{\rho, Q}^{c}+J_{\rho, Q}^{p h},
$$

holds where $J_{\rho, Q}^{c}$ and $J_{\rho, Q}^{p h}$ are given by (3.31) and (3.32).

In particular, let $\left\{S_{c}(\lambda)\right\}_{\lambda \in \mathbb{R}},\left\{\rho_{a c}(\lambda)\right\}_{\lambda \in \mathbb{R}}$ and $\left\{Q_{a c}(\lambda)\right\}_{\lambda \in \mathbb{R}}$ be scattering, density and charge matrices of $S_{c}, \rho$ and $Q$ with respect to $\Pi\left(H_{0}^{a c}\right)$ and let $\left\{S_{p h}(\lambda)\right\}_{\lambda \in \mathbb{R}},\left\{\rho_{a c}^{c}(\lambda)\right\}_{\lambda \in \mathbb{R}}$ and $\left\{Q_{a c}^{c}(\lambda)\right\}_{\lambda \in \mathbb{R}}$ be the scattering, density and charge matrices of the scattering operator $S_{p h}$, density operator $\rho^{c}, c f$. (3.29), and charge operator $Q^{c}, c f$. (3.30), with respect to the spectral representation $\Pi\left(H_{c}^{a c}\right\}$. Then, the following representations:

$$
\begin{aligned}
J_{\rho, Q}^{c} & :=\frac{1}{2 \pi} \int_{\mathbb{R}} \operatorname{tr}\left(\rho_{a c}(\lambda)\left(Q_{a c}(\lambda)-S_{c}(\lambda)^{*} Q_{a c}(\lambda) S_{c}(\lambda)\right) d \lambda,\right. \\
J_{\rho, Q}^{p h} & :=\frac{1}{2 \pi} \int_{\mathbb{R}} \operatorname{tr}\left(\rho_{a c}^{c}(\lambda)\left(Q_{a c}^{c}(\lambda)-S_{p h}(\lambda)^{*} Q_{a c}^{c}(\lambda) S_{p h}(\lambda)\right)\right) d \lambda,
\end{aligned}
$$

take place. 
Proof. Since $\mathcal{S}_{c}$ and $\mathcal{S}_{p h}$ are $\mathfrak{L}_{1}$-scattering systems from Theorem 3.1 the representations (3.35) and (3.36) are easily follow. Taking into account (3.33), we get the following:

$$
\begin{aligned}
& \operatorname{tr}\left(\rho_{a c}^{c}(\lambda)\left(Q_{a c}^{c}(\lambda)-S_{p h}(\lambda)^{*} Q_{a c}^{c}(\lambda) S_{p h}(\lambda)\right)\right)= \\
& \quad \operatorname{tr}\left(W_{-}(\lambda) \rho_{a c} W_{-}(\lambda)^{*}\left(W_{+}(\lambda) Q_{a c}(\lambda) W_{+}(\lambda)-S_{p h}(\lambda)^{*} Q_{a c}^{c}(\lambda) S_{p h}(\lambda)\right)\right) .
\end{aligned}
$$

Using $S_{c}(\lambda)=W_{+}(\lambda)^{*} W_{-}(\lambda)$ we find that:

$$
\begin{aligned}
& \operatorname{tr}\left(\rho_{a c}^{c}(\lambda)\left(Q_{a c}^{c}(\lambda)-S_{p h}(\lambda)^{*} Q_{a c}^{c}(\lambda) S_{p h}(\lambda)\right)\right)=\operatorname{tr}\left(\rho_{a c}(\lambda) \times\right. \\
& \left.\quad\left(S_{c}(\lambda)^{*} Q_{a c}(\lambda) S_{c}(\lambda)-W_{-}(\lambda)^{*} S_{p h}(\lambda)^{*} W_{+}(\lambda) Q_{a c}(\lambda) W_{+}(\lambda)^{*} S_{p h}(\lambda) W_{-}(\lambda)\right)\right) .
\end{aligned}
$$

Since $\left\{H_{c}, H_{0}\right\}$ and $\left\{H, H_{c}\right\}$ are $\mathfrak{L}_{1}$-scattering systems, the existence of the wave operators $W_{ \pm}\left(H, H_{c}\right)$ and $W_{ \pm}\left(H_{c}, H_{0}\right)$ follows. Using the chain rule, we find $W_{ \pm}\left(H, H_{0}\right)=$ $W_{ \pm}\left(H, H_{c}\right) W_{ \pm}\left(H_{c}, H_{0}\right)$ which yields:

$$
\begin{aligned}
S & =W_{+}\left(H, H_{0}\right)^{*} W_{+}\left(H, H_{0}\right) \\
& =W_{+}\left(H_{c}, H_{0}\right)^{*} W_{+}\left(H, H_{c}\right) W_{-}\left(H, H_{c}\right) W_{-}\left(H_{c}, H_{0}\right)=W_{+}\left(H_{c}, H_{0}\right)^{*} S_{p h} W_{-}\left(H_{c}, H_{0}\right) .
\end{aligned}
$$

Hence, the scattering matrix $\{S(\lambda)\}_{\lambda \in \mathbb{R}}$ of $\left\{H, H_{0}\right\}$ admits the representation

$$
S(\lambda)=W_{+}(\lambda)^{*} S_{p h}(\lambda) W_{-}(\lambda), \quad \lambda \in \mathbb{R} .
$$

Inserting (3.38) into (3.37), we get the following:

$$
J_{\rho, Q}^{p h}=\frac{1}{2 \pi} \int_{\mathbb{R}} \operatorname{tr}\left(\rho_{a c}(\lambda)\left(S_{c}(\lambda)^{*} Q_{a c}(\lambda) S_{c}(\lambda)-S(\lambda)^{*} Q_{a c}(\lambda) S(\lambda)\right)\right) d \lambda
$$

Using (3.39), we obtain the following:

$$
J_{\rho, Q}^{c}+J_{\rho, Q}^{p h}=\frac{1}{2 \pi} \int_{\mathbb{R}} \operatorname{tr}\left(\rho_{a c}(\lambda)\left(Q_{a c}(\lambda)-S(\lambda)^{*} Q_{a c}(\lambda) S(\lambda)\right)\right) d \lambda .
$$

Finally, taking into account (3.4), we obtain (3.34).

\section{Remark 3.5.}

(i) The current $J_{\rho, Q}^{c}$ is due to the coupling of the leads to the quantum dot and it is therefore called the contact induced current.

(ii) The current $J_{\rho, Q}^{p h}$ is due to the interaction of photons with electrons and it is called the photon induced current. Notice the this current is calculated under the assumption that the leads are already in contact with the dot.

Corollary 3.6. Let the assumptions of Proposition 3.4 be satisfied. With respect to the spectral representation $\Pi\left(H_{0}^{a c}\right)$ of $H_{0}^{a c}$ the photon induced current $J_{\rho, Q}^{p h}$ can be represented by:

$$
J_{\rho, Q}^{p h}:=\frac{1}{2 \pi} \int_{\mathbb{R}} \operatorname{tr}\left(\widehat{\rho}_{a c}(\lambda)\left(Q_{a c}(\lambda)-\widehat{S}_{p h}(\lambda)^{*} Q_{a c}(\lambda) \widehat{S}_{p h}(\lambda)\right)\right) d \lambda,
$$

where the measurable families $\left\{\widehat{S}_{p h}(\lambda)\right\}_{\lambda \in \mathbb{R}}$ and $\left\{\widehat{\rho}_{a c}(\lambda)\right\}_{\lambda \in \mathbb{R}}$ are given by (3.28) and

$$
\widehat{\rho}_{a c}(\lambda):=S_{c}(\lambda) \rho_{a c}(\lambda) S_{c}(\lambda)^{*} \quad \lambda \in \mathbb{R},
$$

respectively.

Proof. Using (3.33) and $S_{c}(\lambda)=W_{+}(\lambda)^{*} W_{-}(\lambda)$, we find:

$$
\begin{aligned}
& \operatorname{tr}\left(\rho_{a c}^{c}(\lambda)\left(Q_{a c}^{c}(\lambda)-S_{p h}(\lambda)^{*} Q_{a c}^{c}(\lambda) S_{p h}(\lambda)\right)\right)= \\
& \quad \operatorname{tr}\left(S_{c}(\lambda) \rho_{a c}(\lambda) S_{c}(\lambda)^{*}\left(Q_{a c}(\lambda)-W_{+}(\lambda)^{*} S_{p h}(\lambda)^{*} W_{+}(\lambda) Q_{a c}(\lambda) W_{+}(\lambda)^{*} S_{p h}(\lambda) W_{+}(\lambda)\right)\right) .
\end{aligned}
$$


Taking into account the representations (3.28) and (3.41), we get the following:

$$
\begin{aligned}
& \operatorname{tr}\left(\rho_{a c}^{c}(\lambda)\left(Q_{a c}^{c}(\lambda)-S_{p h}(\lambda)^{*} Q_{a c}^{c}(\lambda) S_{p h}(\lambda)\right)\right)= \\
& \quad \operatorname{tr}\left(S_{c}(\lambda) \rho_{a c}(\lambda) S_{c}(\lambda)^{*}\left(Q_{a c}(\lambda)-\widehat{S}_{p h}(\lambda)^{*} Q_{a c}(\lambda) \widehat{S}_{p h}(\lambda)\right)\right),
\end{aligned}
$$

which immediately yields (3.40).

Remark 3.7. In the following, we call $\left\{\hat{\rho}_{a c}(\lambda)\right\}_{\lambda \in \mathbb{R}}$, cf. (3.41), the photon modified electron density matrix. Notice that $\left\{\widehat{\rho}_{a c}(\lambda)\right\}_{\lambda \in \mathbb{R}}$ might be non-diagonal, even if the electron density matrix $\left\{\rho_{a c}(\lambda)\right\}_{\lambda \in \mathbb{R}}$ is diagonal.

\section{Analysis of currents}

In the following, we analyze currents $J_{\rho, Q}^{c}$ and $J_{\rho, Q}^{p h}$ under the assumption that $\rho$ and $Q$ have the tensor product structure:

$$
\rho=\rho^{e l} \otimes \rho^{p h} \quad \text { and } \quad Q=q^{e l} \otimes q^{p h},
$$

where $\rho^{e l}$ and $\rho^{p h}$ as well as $q^{e l}$ and $q^{p h}$ are density operators and (tempered) charges for $h_{0}^{e l}$ and $h^{p h}$, respectively. Since $\rho^{p h}$ commutes with $h^{p h}$, which is discrete, the operator $\rho^{p h}$ has the form:

$$
\rho^{p h}=\sum_{n \in \mathbb{N}_{0}} \rho^{p h}(n)\left(\cdot, \Upsilon_{n}\right) \Upsilon_{n}
$$

where $\rho^{p h}(n)$ are non-negative numbers. Similarly, $q^{p h}$ can be represented as:

$$
q^{p h}=\sum_{n \in \mathbb{N}_{0}} q^{p h}(n)\left(\cdot, \Upsilon_{n}\right) \Upsilon_{n}
$$

where $q^{p h}(n)$ are real numbers.

Lemma 4.1. Let $\mathcal{S}=\left\{H, H_{0}\right\}$ be the JCL-model. Assume that $\rho \neq 0$ and $Q$ have the structure (4.1) where $\rho^{\text {el }}$ is a density operator and $q^{\text {el }}$ is a charge for $h_{0}^{e l}$.

(i) The operator $\left(H_{0}-i\right)^{p+2} \rho, p \in \mathbb{N}_{0}$, is bounded if and only if the condition:

$$
\sup _{n \in \mathbb{N}_{0}} \rho^{p h}(n) n^{p+2}<\infty
$$

is satisfied.

(ii) The charge $Q$ is p-tempered if and only if:

$$
\sup _{n \in \mathbb{N}}\left|q^{p h}(n)\right| n^{-p}<\infty,
$$

is valid

Proof. (i) The operator $\left(H_{0}-i\right)^{p+2} \rho$ admits the representation:

$$
\left(H_{0}-i\right)^{p+2} \rho=\bigoplus_{p \in \mathbb{N}_{0}} \rho^{p h}(n)\left(h_{0}^{e l}+n \omega-i\right)^{p+2} \rho^{e l} .
$$

We have:

$$
\begin{aligned}
\left\|\left(H_{0}-i\right)^{p+2} \rho\right\|_{\mathfrak{L}(\mathfrak{H})} & =\sup _{p \in \mathbb{N}_{0}} \rho^{p h}(n)\left\|\left(h_{0}^{e l}+n \omega-i\right)^{p+2} \rho^{e l}\right\|_{\mathfrak{L}\left(\mathfrak{h}^{e l}\right)} \\
& =\sup _{p \in \mathbb{N}_{0}} \rho^{p h}(n) n^{p+2} n^{-(p+2)}\left\|\left(h_{0}^{e l}+n \omega-i\right)^{p+2} \rho^{e l}\right\|_{\left.\mathfrak{L}(\mathfrak{h})^{e l}\right)} .
\end{aligned}
$$

Since $\lim _{n \rightarrow \infty} n^{-(p+2)}\left\|\left(h_{0}^{e l}+n \omega-i\right)^{p+2} \rho^{e l}\right\|_{\mathfrak{L}\left(\mathfrak{h}^{e l}\right)}=\omega^{p+2}\left\|\rho^{e l}\right\|_{\mathfrak{L}\left(\mathfrak{h}^{e l}\right)}$, we obtain for sufficiently large $n \in \mathbb{N}_{0}$ that:

$$
\frac{\omega^{p+2}}{2}\left\|\rho^{e l}\right\|_{\mathfrak{L}\left(\mathfrak{h}^{e l}\right)} \leqslant n^{-(p+2)}\left\|\left(h_{0}^{e l}+n \omega-i\right)^{p+2} \rho^{e l}\right\|_{\mathfrak{L}\left(\mathfrak{h}^{e l}\right)}
$$


Using that and (4.6), we immediately obtain (4.4). Conversely, from (4.6) and (4.4), we obtain that $\left(H_{0}-i\right)^{p+2} \rho$ is a bounded operator.

(ii) As above, we have:

$$
Q\left(H_{0}-i\right)^{-p}=\bigoplus_{n \in \mathbb{N}_{0}} q^{p h}(n) q^{e l}
$$

Hence:

$$
\left\|Q\left(H_{0}-i\right)^{-p}\right\|_{\mathfrak{L}(\mathfrak{H})}=\sup _{n \in \mathbb{N}_{0}}\left|q^{p h}(n)\right|\left\|q^{e l}\left(h_{0}^{e l}+n \omega-i\right)^{-p}\right\|_{\mathfrak{L}\left(\mathfrak{h}^{e l}\right)} .
$$

Since $\lim _{n \rightarrow \infty} n^{p}\left\|\left(h_{0}^{e l}+n \omega-i\right)^{-p}\right\|_{\mathfrak{L}\left(\mathfrak{h}^{e l}\right)}=\omega^{-p}\left\|q^{e l}\right\|_{\mathfrak{L}\left(\mathfrak{h}^{e l}\right)}$, we similarly obtain, as above, that (4.5) holds. The converse is obvious.

\subsection{Contact induced current}

Let us recall that $\mathcal{S}_{c}=\left\{H_{c}, H_{0}\right\}$ is a $\mathfrak{L}_{1}$-scattering system. Straightforward computation shows that:

$$
W_{ \pm}\left(H_{c}, H_{0}\right)=w_{ \pm}\left(h^{e l}, h_{0}^{e l}\right) \otimes I_{\mathfrak{h}^{p h}},
$$

where $w_{ \pm}\left(h^{e l}, h_{0}^{e l}\right)$ is given by (3.24). Hence:

$$
S_{c}=s_{c} \otimes I_{\mathfrak{h}^{p h}}, \quad \text { where } \quad s_{c}:=w_{+}\left(h_{c}^{e l}, h_{0}^{e l}\right)^{*} w_{-}\left(h_{c}^{e l}, h_{0}^{e l}\right) .
$$

Proposition 4.2. Let $\mathcal{S}=\left\{H, H_{0}\right\}$ be the JCL-model. Assume that $\rho$ and $Q$ are given by (4.1) where $\rho^{e l}$ and $q^{e l}$ are density and charge operators for $h_{0}^{e l}$ and $\rho^{\text {ph }}$ and $q^{\text {ph }}$ for $h^{p h}$, respectively. If for some $p \in \mathbb{N}_{0}$ the conditions (4.4) and (4.5) are satisfied, then the current $J_{\rho, Q}^{c}$ is well defined and admits the representation:

$$
J_{\rho, Q}^{c}=\gamma J_{\rho^{e l}, q^{e l}}^{s_{c}}, \quad \gamma:=\sum_{n \in \mathbb{N}_{0}} q^{p h}(n) \rho^{p h}(n),
$$

where $J_{\rho^{e l}, q^{e l}}^{s_{c}}$ is defined by (3.2). In particular, if $\operatorname{tr}\left(\rho^{p h}\right)=1$ and $q^{p h}=I_{\mathfrak{h}^{p h}}$, then $J_{\rho, Q}^{c}=J_{\rho^{e l}, q^{e l}}^{s_{c}}$.

Proof. First, we note that by lemma 4.1 the operator $\left(H_{0}-i\right)^{p+2} \rho$ is bounded and $Q$ is $p$-tempered. Hence, the current $J_{\rho, Q}^{S_{c}}$ is correctly defined and the Landauer-Büttiker formula (3.4) is valid.

With respect to the spectral representation $\Pi\left(H_{0}^{a c}\right)$ of Lemma 2.12, the charge matrix $\left\{Q_{a c}(\lambda)\right\}_{\lambda \in \mathbb{R}}$ of $Q_{a c}=q_{a c}^{e l} \otimes q^{p h}$ admits the representation:

$$
Q_{a c}(\lambda)=\bigoplus_{n \in \mathbb{N}_{0}} q_{a c}^{e l}(\lambda-n \omega) q^{p h}(n), \quad \lambda \in \mathbb{R} .
$$

Since $S_{c}=s_{c} \otimes I_{\mathfrak{h}^{p h}}$, the scattering matrix $\left\{S_{c}(\lambda)\right\}_{\lambda \in \mathbb{R}}$ admits the representation:

$$
S_{c}(\lambda)=\bigoplus_{n \in \mathbb{N}_{0}} s_{c}(\lambda-n \omega), \quad \lambda \in \mathbb{R} .
$$

Hence:

$$
\begin{aligned}
& Q_{a c}(\lambda)-S_{c}(\lambda)^{*} Q_{a c}(\lambda) S_{c}(\lambda)= \\
& \bigoplus_{n \in \mathbb{N}_{0}} q^{p h}(n)\left(q_{a c}^{e l}(\lambda-n \omega)-s_{c}(\lambda-n \omega)^{*} q_{a c}^{e l}(\lambda-\omega n) s_{c}(\lambda-n \omega)\right) .
\end{aligned}
$$

Moreover, the density matrix $\left\{\rho_{a c}(\lambda)\right\}_{\lambda \in \mathbb{R}}$ admits the representation:

$$
\rho_{a c}(\lambda)=\bigoplus_{n \in \mathbb{N}_{0}} \rho^{p h}(n) \rho_{a c}^{e l}(\lambda-n \omega)
$$


Inserting (4.10) into (4.9) we find the following:

$$
\begin{aligned}
& \rho^{a c}(\lambda)\left(Q_{a c}(\lambda)-S_{c}(\lambda)^{*} Q_{a c}(\lambda) S_{c}(\lambda)\right)= \\
& \bigoplus_{n \in \mathbb{N}_{0}} q^{p h}(n) \rho^{p h}(n) \rho_{a c}^{e l}(\lambda-n \omega)\left(q_{a c}^{e l}(\lambda-\omega n)-s_{c}(\lambda-n \omega)^{*} q_{a c}^{e l}(\lambda-\omega n) s_{c}(\lambda-n \omega)\right)
\end{aligned}
$$

Since $\gamma=\sum_{n \in \mathbb{N}_{0}} q^{p h}(n) \rho^{p h}(n)$ is absolutely convergent by (4.4) and (4.5), we obtain that:

$$
\begin{aligned}
& \operatorname{tr}\left(\rho^{a c}(\lambda)\left(Q_{a c}(\lambda)-S_{c}(\lambda)^{*} Q_{a c}(\lambda) S_{c}(\lambda)\right)\right)= \\
& \quad \sum_{n \in \mathbb{N}_{0}} q^{p h}(n) \rho^{p h}(n) \operatorname{tr}\left(\rho_{a c}^{e l}(\lambda-n \omega)\left(q_{a c}^{e l}(\lambda-\omega n)-s_{c}(\lambda-n \omega)^{*} q_{a c}^{e l}(\lambda-\omega n) s_{c}(\lambda-n \omega)\right)\right)
\end{aligned}
$$

Clearly, we have:

$$
\begin{aligned}
& \left|\operatorname{tr}\left(\rho_{a c}^{e l}(\lambda-n \omega)\left(q_{a c}^{e l}(\lambda-\omega n)-s_{c}(\lambda-n \omega)^{*} q_{a c}^{e l}(\lambda-\omega n) s_{c}(\lambda-n \omega)\right)\right)\right| \leqslant \\
& 4\left\|\rho_{a c}^{e l}(\lambda-n \omega)\right\|_{\mathfrak{L}\left(\mathfrak{h}_{n}(\lambda)\right)}\left\|q_{a c}^{e l}(\lambda-n \omega)\right\|_{\mathfrak{L}\left(\mathfrak{h}_{n}(\lambda)\right)}, \quad \lambda \in \mathbb{R} .
\end{aligned}
$$

We insert (4.11) into the Landauer-Büttiker formula (3.35). Using (4.4) and (4.5) as well as

$$
\int_{\mathbb{R}}\left\|\rho_{a c}^{e l}(\lambda)\right\|_{\mathfrak{L}\left(\mathfrak{h}_{n}(\lambda)\right)}\left\|q_{a c}^{e l}(\lambda)\right\|_{\mathfrak{L}\left(\mathfrak{h}_{n}(\lambda)\right)} d \lambda<\infty,
$$

we see that we can interchange the integral and the sum. By doing so, we get:

$$
\begin{aligned}
J_{\rho, Q}^{c} & =\sum_{n \in \mathbb{N}_{0}} q^{p h}(n) \rho^{p h}(n) \frac{1}{2 \pi} \int_{\mathbb{R}} \operatorname{tr}\left(\rho_{a c}^{e l}(\lambda-n \omega) \times\right. \\
& \left.\left(q_{a c}^{e l}(\lambda-\omega n)-s_{c}(\lambda-n \omega)^{*} q_{a c}^{e l}(\lambda-\omega n) s_{c}(\lambda-n \omega)\right)\right) d \lambda .
\end{aligned}
$$

Using (3.25) we prove (4.7).

If $\operatorname{tr}\left(\rho^{p h}\right)=1$, then $\sum_{\mathbb{N}_{0}} \rho^{p h}(n)=1$. Furthermore, if $\rho^{p h}=I_{\mathfrak{h}^{p h}}$, then $q^{p h}(n)=1$. Hence, $\gamma=1$.

\subsection{Photon induced current}

To calculate the current $J_{\rho, Q}^{p h}$, we use representation (3.40). We then set:

$$
\widehat{S}_{m n}^{p h}(\lambda):=P_{m}(\lambda) \widehat{S}_{p h}(\lambda)\left\lceil\mathfrak{h}_{n}(\lambda), \quad \lambda \in \mathbb{R},\right.
$$

where $\left\{\widehat{S}_{p h}(\lambda)\right\}_{\lambda \in \mathbb{R}}$ is defined by (3.28) and $P_{m}(\lambda)$ is the orthogonal projection from $\mathfrak{h}(\lambda)$, cf. (2.37), onto $\mathfrak{h}_{m}(\lambda):=\mathfrak{h}^{e l}(\lambda-m \omega), \lambda \in \mathbb{R}$.

Proposition 4.3. Let $\mathcal{S}=\left\{H, H_{0}\right\}$ be the JCL-model. Assume that $\rho$ and $Q$ are given by (4.1) where $\rho^{e l}$ and $q^{e l}$ are density and charge operators for $h_{0}^{e l}$ and $\rho^{\text {ph }}$ and $q^{\text {ph }}$ for $h^{p h}$, respectively. If for some $p \in \mathbb{N}_{0}$ the conditions (4.4) and (4.5) are satisfied, then the current $J_{\rho, Q}^{p h}$ is well-defined and it admits the following representation:

$$
\begin{aligned}
J_{\rho, Q}^{p h}= & \sum_{m \in \mathbb{N}_{0}} \rho^{p h}(m) \sum_{n \in \mathbb{N}_{0}} q^{p h}(n) \frac{1}{2 \pi} \int_{\mathbb{R}} d \lambda \operatorname{tr}\left(\widehat{\rho}_{a c}^{e l}(\lambda-m \omega) \times\right. \\
& \left.\left(q_{a c}^{e l}(\lambda-n \omega) \delta_{m n}-\widehat{S}_{n m}^{p h}(\lambda)^{*} q_{a c}^{e l}(\lambda-n \omega) \widehat{S}_{n m}^{p h}(\lambda)\right)\right),
\end{aligned}
$$

where $\left\{\widehat{\rho}_{a c}^{e l}(\lambda)\right\}_{\lambda \in \mathbb{R}}$ is the photon modified electron density defined, cf. (3.41), which takes the following form:

$$
\widehat{\rho}_{a c}^{e l}(\lambda)=s_{c}(\lambda) \rho^{e l}(\lambda) s_{c}(\lambda)^{*}, \quad \lambda \in \mathbb{R} .
$$


Proof. By Lemma 4.1 we get that that the charge $Q$ is $p$-tempered and $\left(H_{0}-i\right)^{p} \rho$ is a bounded operator. By Corollary 3.2, the current $J_{\rho, Q}^{p h}:=J_{\rho^{c}, Q^{c}}^{S_{p h}}$ is well-defined.

Since $\left(Q_{a c}(\lambda)-\widehat{S}_{p h}(\lambda)^{*} Q_{a c}(\lambda) \widehat{S}_{p h}(\lambda)\right)$ is a trace class operator for $\lambda \in \mathbb{R}$, we get from (3.40) and (4.10) that:

$$
\begin{aligned}
& \operatorname{tr}\left(\widehat{\rho}_{a c}(\lambda)\left(Q_{a c}(\lambda)-\widehat{S}_{p h}(\lambda)^{*} Q_{a c}(\lambda) \widehat{S}_{p h}(\lambda)\right)\right)= \\
& \quad \sum_{m \in \mathbb{N}_{0}} \rho^{p h}(m) \operatorname{tr}\left(\widehat{\rho}^{e l}(\lambda-m \omega) P_{m}(\lambda)\left(Q_{a c}(\lambda)-\widehat{S}_{p h}(\lambda)^{*} Q_{a c}(\lambda) \widehat{S}_{p h}(\lambda)\right) P_{m}(\lambda)\right)
\end{aligned}
$$

Furthermore, we have:

$$
\begin{aligned}
P_{m}(\lambda) & \left(Q_{a c}(\lambda)-\widehat{S}_{p h}(\lambda)^{*} Q_{a c}(\lambda) \widehat{S}_{p h}(\lambda)\right) P_{m}(\lambda) \\
& =q^{p h}(m)\left(q^{e l}(\lambda-m \omega)-P_{m}(\lambda) \widehat{S}_{p h}(\lambda)^{*} Q_{a c}(\lambda) \widehat{S}_{p h}(\lambda)\right) P_{m}(\lambda) \\
& =q^{p h}(m) q^{e l}(\lambda-m \omega)-\sum_{n \in \mathbb{N}_{0}} q^{p h}(n) \widehat{S}_{n m}^{p h}(\lambda)^{*} q^{e l}(\lambda-n \omega) \widehat{S}_{n m}^{p h}(\lambda)
\end{aligned}
$$

for $\lambda \in \mathbb{R}$ where $\widehat{S}_{n m}^{p h}(\lambda)^{*}:=P_{n}(\lambda) \widehat{S}_{p h}(\lambda) P_{m}(\lambda), \lambda \in \mathbb{R}$. Notice that $\sum_{n \in \mathbb{N}_{0}}$ is a sum with a finite number of summands. Hence:

$$
\begin{gathered}
\operatorname{tr}\left(\widehat{\rho}_{a c}(\lambda)\left(Q_{a c}(\lambda)-\widehat{S}_{p h}(\lambda)^{*} Q_{a c}(\lambda) \widehat{S}_{p h}(\lambda)\right)\right)=\sum_{m \in \mathbb{N}_{0}} \rho^{p h}(m) \sum_{n \in \mathbb{N}_{0}} q^{p h}(n) \times \\
\quad \operatorname{tr}\left(\widehat{\rho}^{e l}(\lambda-m \omega)\left(q^{e l}(\lambda-m \omega) \delta_{m n}-\widehat{S}_{n m}^{p h}(\lambda)^{*} q^{e l}(\lambda-n \omega) \widehat{S}_{n m}^{p h}(\lambda)\right)\right)
\end{gathered}
$$

We are going to show that

$$
\begin{aligned}
\sum_{m \in \mathbb{N}_{0}} \rho^{p h}(m) & \sum_{n \in \mathbb{N}_{0}}\left|q^{p h}(n)\right| \int_{\mathbb{R}} \mid \operatorname{tr}\left(\widehat{\rho}^{e l}(\lambda-m \omega) \times\right. \\
& \left.\left(q^{e l}(\lambda-m \omega) \delta_{m n}-\widehat{S}_{n m}^{p h}(\lambda)^{*} q^{e l}(\lambda-n \omega) \widehat{S}_{n m}^{p h}(\lambda)\right)\right) \mid d \lambda<\infty .
\end{aligned}
$$

Clearly, one has the following estimate:

$$
\begin{aligned}
& \left|\operatorname{tr}\left(\widehat{\rho}^{e l}(\lambda-m \omega)\left(q^{e l}(\lambda-m \omega) \delta_{m n}-\widehat{S}_{n m}^{p h}(\lambda)^{*} q^{e l}(\lambda-n \omega) \widehat{S}_{n m}^{p h}(\lambda)\right)\right)\right| \leqslant \\
& 2\left\|\widehat{\rho}^{e l}(\lambda-m \omega)\right\|_{\mathfrak{L}\left(\mathfrak{h}_{m}(\lambda)\right)}\left(\left\|q^{e l}(\lambda-m \omega)\right\|_{\mathfrak{L}\left(\mathfrak{h}_{m}(\lambda)\right)} \delta_{n m}+\left\|q^{e l}(\lambda-n \omega)\right\|_{\mathfrak{L}\left(\mathfrak{h}_{n}(\lambda)\right)}\right) .
\end{aligned}
$$

Furthermore, we get:

$$
\begin{array}{r}
\int_{\lambda \in \mathbb{R}}\left\|\hat{\rho}^{e l}(\lambda-m \omega)\right\|_{\mathfrak{L}\left(\mathfrak{h}_{m}(\lambda)\right)}\left\|q^{e l}(\lambda-m \omega)\right\|_{\mathfrak{L}\left(\mathfrak{h}_{m}(\lambda)\right)} \delta_{n m} \leqslant \\
\int_{\lambda \in \mathbb{R}}\left\|\hat{\rho}^{e l}(\lambda)\right\|_{\mathfrak{L}\left(\mathfrak{h}_{m}(\lambda)\right)}\left\|q^{e l}(\lambda)\right\|_{\mathfrak{L}\left(\mathfrak{h}_{m}(\lambda)\right)} d \lambda
\end{array}
$$

and

$$
\begin{aligned}
\int_{\mathbb{R}} \| \hat{\rho}^{e l}(\lambda-m \omega) & \left\|_{\mathfrak{L}\left(\mathfrak{h}_{m}(\lambda)\right)}\right\| q^{e l}(\lambda-n \omega) \|_{\mathfrak{L}\left(\mathfrak{h}_{n}(\lambda)\right)} d \lambda \leqslant \\
& \left\|q_{a c}^{e l}\right\|_{\mathfrak{L}\left(\mathfrak{h}^{e l}\right)} \int_{\lambda \in \mathbb{R}}\left\|\hat{\rho}^{e l}(\lambda-(m-n) \omega)\right\|_{\mathfrak{L}\left(\mathfrak{h}_{m-n}(\lambda)\right)} d \lambda
\end{aligned}
$$

If the conditions (4.4) and (4.5) are satisfied, then

$$
\sum_{m \in \mathbb{N}_{0}} \rho^{p h}(m)\left|q^{p h}(m)\right| \int_{\mathbb{R}}\left\|\widehat{\rho}^{e l}(\lambda)\right\|_{\mathfrak{L}\left(\mathfrak{h}_{m}(\lambda)\right)}\left\|q^{e l}(\lambda)\right\|_{\mathfrak{L}\left(\mathfrak{h}_{m}(\lambda)\right)} d \lambda<\infty .
$$


Furthermore, we obtain:

$$
\begin{aligned}
& \sum_{m \in \mathbb{N}_{0}} \rho^{p h}(m) \sum_{n \in \mathbb{N}_{0}}\left|q^{p h}(n)\right| \int_{\lambda \in \mathbb{R}}\left\|\hat{\rho}^{e l}(\lambda-(m-n) \omega)\right\|_{\mathfrak{L}\left(\mathfrak{h}_{m-n}(\lambda)\right)} d \lambda \leqslant \\
& \left(v_{\max }-v_{\text {min }}+4\right)\left\|\rho_{a c}^{e l}\right\|_{\mathfrak{L}\left(\mathfrak{h}^{e l}\right)} \sum_{m \in \mathbb{N}_{0}} \rho^{p h}(m) \sum_{|m-n| \leqslant d_{\text {max }}}\left|q^{p h}(n)\right|<\infty,
\end{aligned}
$$

where $d_{\max }$ is introduced by Lemma 2.12. To prove the following:

$$
\sum_{m \in \mathbb{N}_{0}} \rho^{p h}(m) \sum_{|m-n| \leqslant d_{\max }}\left|q^{p h}(n)\right|<\infty,
$$

we again use (4.4) and (4.5). The last step allows us to interchange the integral and the sums, which immediately proves (4.12).

Corollary 4.4. Let $\mathcal{S}=\left\{H, H_{0}\right\}$ be the JCL-model. We assume that $\rho$ and $Q$ are given by (4.1), where $\rho^{e l}$ and $q^{\text {el }}$ are density and charge operators for $h_{0}^{e l}$ and $\rho^{\text {ph }}$ and $q^{p h}$ for $h^{p h}$, respectively. If $\rho^{e l}$ is an equilibrium state, i.e. $\rho^{e l}=f^{e l}\left(h_{0}^{e l}\right)$, then:

$$
\begin{aligned}
J_{\rho, Q}^{p h}= & \sum_{m, n \in \mathbb{N}_{0}} q^{p h}(n) \frac{1}{2 \pi} \int_{\mathbb{R}}\left(\rho^{p h}(n) f^{e l}(\lambda-n \omega)-\rho^{p h}(m) f^{e l}(\lambda-m \omega)\right) \times \\
& \operatorname{tr}\left(\widehat{S}_{n m}^{p h}(\lambda)^{*} q_{a c}^{e l}(\lambda-n \omega) \widehat{S}_{n m}^{p h}(\lambda)\right) d \lambda .
\end{aligned}
$$

Proof. From (4.12), we obtain the following:

$$
\begin{aligned}
J_{\rho, Q}^{p h}= & \sum_{n \in \mathbb{N}_{0}} q^{p h}(n) \sum_{m \in \mathbb{N}_{0}} \rho^{p h}(m) \frac{1}{2 \pi} \int_{\mathbb{R}} d \lambda f^{e l}(\lambda-m \omega) \times \\
& \operatorname{tr}\left(q_{a c}^{e l}(\lambda-n \omega) \delta_{m n}-\widehat{S}_{n m}^{p h}(\lambda)^{*} q_{a c}^{e l}(\lambda-n \omega) \widehat{S}_{n m}^{p h}(\lambda)\right) .
\end{aligned}
$$

Hence,

$$
\begin{gathered}
J_{\rho, Q}^{p h}=\sum_{n \in \mathbb{N}_{0}} q^{p h}(n) \frac{1}{2 \pi} \int_{\mathbb{R}} d \lambda \sum_{m \in \mathbb{N}_{0}} \rho^{p h}(m) f^{e l}(\lambda-m \omega) \times \\
\operatorname{tr}\left(q_{a c}^{e l}(\lambda-n \omega) \delta_{m n}-\widehat{S}_{n m}^{p h}(\lambda)^{*} q_{a c}^{e l}(\lambda-n \omega) \widehat{S}_{n m}^{p h}(\lambda)\right) .
\end{gathered}
$$

This gives the following:

$$
\begin{aligned}
J_{\rho, Q}^{p h}= & \sum_{n \in \mathbb{N}_{0}} q^{p h}(n) \frac{1}{2 \pi} \int_{\mathbb{R}} d \lambda\left(\rho^{p h}(n) f^{e l}(\lambda-n \omega) \operatorname{tr}\left(q_{a c}^{e l}(\lambda-n \omega)\right)-\right. \\
& \left.\sum_{m \in \mathbb{N}_{0}} \rho^{p h}(m) f^{e l}(\lambda-m \omega) \operatorname{tr}\left(\widehat{S}_{n m}^{p h}(\lambda)^{*} q_{a c}^{e l}(\lambda-n \omega) \widehat{S}_{n m}^{p h}(\lambda)\right)\right) .
\end{aligned}
$$

Since

$$
\begin{aligned}
& \sum_{m \in \mathbb{N}_{0}} \rho^{p h}(m) f^{e l}(\lambda-m \omega) \operatorname{tr}\left(\widehat{S}_{n m}^{p h}(\lambda)^{*} q_{a c}^{e l}(\lambda-n \omega) \widehat{S}_{n m}^{p h}(\lambda)\right)= \\
& \sum_{m \in \mathbb{N}_{0}}\left(\rho^{p h}(m) f^{e l}(\lambda-m \omega)-\rho^{p h}(n) f^{e l}(\lambda-n \omega)\right) \operatorname{tr}\left(\widehat{S}_{n m}^{p h}(\lambda)^{*} q_{a c}^{e l}(\lambda-n \omega) \widehat{S}_{n m}^{p h}(\lambda)\right)+ \\
& \rho^{p h}(n) f^{e l}(\lambda-n \omega) \sum_{m \in \mathbb{N}_{0}} \operatorname{tr}\left(\widehat{S}_{n m}^{p h}(\lambda)^{*} q_{a c}^{e l}(\lambda-n \omega) \widehat{S}_{n m}^{p h}(\lambda)\right),
\end{aligned}
$$

then inserting this into (4.15) we obtain (4.14). 


\section{Electron and photon currents}

\subsection{Electron current}

To calculate the electron current induced by contacts and photon contact, we make the following choice throughout this section. We set

$$
Q_{\alpha}^{e l}:=q_{\alpha}^{e l} \otimes q^{p h}, \quad q_{\alpha}^{e l}:=-\mathfrak{e} p_{\alpha}^{e l} \quad \text { and } \quad q^{p h}:=I_{\mathfrak{h}^{p h}}, \quad \alpha \in\{l, r\},
$$

where $p_{\alpha}^{e l}$ denotes the orthogonal projection from $\mathfrak{h}^{\text {el }}$ onto $\mathfrak{h}_{\alpha}^{e l}$. By $\mathfrak{e}>0$, we denote the magnitude of the elementary charge. Since $p_{\alpha}^{e l}$ commutes with $h_{\alpha}^{e l}$, one can easily verify that $Q_{\alpha}^{e l}$ commutes with $H_{0}$, which shows that $Q_{\alpha}^{e l}$ is a charge. Following [1], the flux related to $Q_{\alpha}^{e l}$ gives us the electron current $J_{\rho, Q_{\alpha}^{e l}}^{S}$ entering the lead $\alpha$ from the sample. Notice $Q_{\alpha}^{e l}=-\mathfrak{e} P_{\alpha}$ where $P_{\alpha}$ is the orthogonal projection from $\mathfrak{H}$ onto $\mathfrak{H}_{\alpha}:=\mathfrak{h}_{\alpha}^{e l} \otimes \mathfrak{h}^{p h}$. Since $q^{p h}=I_{\mathfrak{h}^{p h}}$, condition (4.5) is immediately satisfied for any $p \geqslant 0$.

Let $f(\cdot): \mathbb{R} \longrightarrow \mathbb{R}$ be a non-negative bounded measurable function. We set:

$$
\rho^{e l}=\rho_{l}^{e l} \oplus \rho_{S}^{e l} \oplus \rho_{r}^{e l}, \quad \rho_{\alpha}^{e l}:=f\left(h_{\alpha}^{e l}-\mu_{\alpha}\right), \quad \alpha \in\{l, r\},
$$

and $\rho=\rho^{e l} \otimes \rho^{p h}$. By $\mu_{\alpha}$ the chemical potential of the lead $\alpha$ is denoted. In applications, one sets $f(\lambda):=f_{F D}(\lambda), \lambda \in \mathbb{R}$, where $f_{F D}(\lambda)$ is the so-called Fermi-Dirac distribution given by (3.21). If $\beta=\infty$, then $f_{F D}(\lambda):=\chi_{\mathbb{R}_{-}}(\lambda), \lambda \in \mathbb{R}$. Notice that $\left[\rho^{e l}, p^{e l}\right]=0$. For $\rho^{p h}$, we choose the Gibbs state:

$$
\rho^{p h}:=\frac{1}{Z} e^{-\beta h^{p h}}, \quad Z=\operatorname{tr}\left(e^{-\beta h^{p h}}\right)=\frac{1}{1-e^{-\beta \omega}} .
$$

Hence, $\rho^{p h}=\left(1-e^{-\beta \omega}\right) e^{-\beta h^{p h}}$. If $\beta=\infty$, then $\rho^{p h}:=\left(\cdot, \Upsilon_{0}\right) \Upsilon_{0}$. Clearly, $\operatorname{tr}\left(\rho^{p h}\right)=1$. We note that $\rho^{p h}(n)=\left(1-e^{-\beta \omega}\right) e^{-n \beta \omega}, n \in \mathbb{N}_{0}$, satisfies the condition (4.4) for any $p \geqslant 0$. Accordingly, $\rho_{0}=\rho^{e l} \otimes \rho^{p h}$ is the density operator for $H_{0}$.

Definition 5.1. Let $\mathcal{S}=\left\{H, H_{0}\right\}$ be the $J C L$-model. If $Q:=Q_{\alpha}^{e l}$, where $Q_{\alpha}^{e l}$ is given by (5.1), and $\rho:=\rho_{0}:=\rho^{e l} \otimes \rho^{p h}$, where $\rho^{e l}$ and $\rho^{p h}$ are given by (5.2) and (5.3), then $J_{\rho_{0}, Q_{\alpha}^{e l}}^{e l}:=J_{\rho_{0}, Q_{\alpha}^{e l}}^{\mathcal{S}}$ is called the electron current entering the lead $\alpha$. The currents $J_{\rho_{0}, Q_{\alpha}^{e l}}^{c}$ and $J_{\rho_{0}, Q_{\alpha}^{e l}}^{p h}$ are called the contact-induced and photon-induced electron currents.

5.1.1. Contact induced electron current. The following proposition immediately follows from Proposition 4.2.

Proposition 5.2. Let $\mathcal{S}=\left\{H, H_{0}\right\}$ be the JCL-model. Then the contact induced electron current $J_{\rho_{0}, Q_{\alpha}^{e l}}^{c l}, \alpha \in\{l, r\}$, is given by $J_{\rho_{0}, Q_{\alpha}^{e l}}^{c}=J_{\rho^{e l}, q_{\alpha}^{e l}}^{s_{c}}$. In particular, one has:

$$
J_{\rho_{0}, Q_{\alpha}^{e l}}^{c}=-\frac{\mathfrak{e}}{2 \pi} \int_{\mathbb{R}}\left(f\left(\lambda-\mu_{\alpha}\right)-f\left(\lambda-\mu_{\varkappa}\right) \sigma_{c}(\lambda) d \lambda, \quad \alpha, \varkappa \in\{l, r\}, \quad \alpha \neq \varkappa,\right.
$$

where $\left\{\sigma_{c}(\lambda)\right\}_{\lambda \in \mathbb{R}}$ is the channel cross-section from left to the right of the scattering system $s_{c}=$ $\left\{h^{\text {el }}, h_{0}^{\text {el }}\right\}$, cf. Example 3.3.

Proof. Since $\operatorname{tr}\left(\rho^{p h}\right)=1$ it follows from Proposition 4.2 that $J_{\rho_{0}, Q_{\alpha}^{e l}}^{c}=J_{\rho^{e l}, q_{\alpha}^{e l}}^{s_{c}}$. From (3.26), cf. Example 3.3, we find (5.4).

If $\mu_{l}>\mu_{r}$ and $f(\cdot)$ is decreasing, then $J_{\rho_{0}, Q_{l}^{e l}}^{c}<0$. Hence, the electron contact current is going from the left lead to the right which is in accordance with the physical expectations. In particular, this is valid for the Fermi-Dirac distribution.

Proposition 5.3. Let $\mathcal{S}=\left\{H, H_{0}\right\}$ be the JCL-model. Further, let $\rho^{\text {el }}$ and $\rho^{\text {ph }}$ be given by (5.2) and (5.3), respectively. If the charge $Q_{\alpha}^{e l}$ is given by (5.1), then the following holds: 
(E) If $\mu_{l}=\mu_{r}$, then $J_{\rho_{0}, Q_{\alpha}^{e l}}^{c}=0, \alpha \in\{l, r\}$.

(S) If $v_{l} \geqslant v_{r}+4$, then $J_{\rho_{0}, Q_{\alpha}^{e l}}^{c}=0, \alpha \in\{l, r\}$, even if $\mu_{l} \neq \mu_{l}$.

(C) If $e_{0}^{S}=\delta_{0}^{S}$ and $e_{1}^{S}=\delta_{1}^{S}$, then $J_{\rho_{0}, Q_{\alpha}^{e l}}^{c}=0, \alpha \in\{l, r\}$, even if $\mu_{l} \neq \mu_{l}$.

Proof. (E) If $\mu_{l}=\mu_{r}$, then $f\left(\lambda-\mu_{l}\right)=f\left(\lambda-\mu_{r}\right)$. Applying formula (5.4) we obtain $J_{\rho_{0}, Q_{\alpha}^{e l}}^{c}=0$.

(S) If $v_{l} \geqslant v_{r}+4$, then $h_{0}^{e l, a c}$ has simple spectrum. Hence the scattering matrix $\left\{s_{c}(\lambda)\right\}_{\lambda \in \mathbb{R}}$ of the scattering system $s_{c}=\left\{h^{e l}, h_{0}^{e l}\right\}$ is a scalar function which immediately yields $\sigma_{c}(\lambda)=0$, $\lambda \in \mathbb{R}$, which yields $J_{\rho_{0}, Q_{\alpha}^{e l}}^{c}=0$.

(C) In this case, the Hamiltonian $h^{e l}$ decomposes into the direct sum of two non-interacting Hamiltonians. Hence, the scattering matrix of $\left\{s_{c}(\lambda)\right\}_{\lambda \in \mathbb{R}}$ of the scattering system $s_{c}=\left\{h^{e l}, h_{0}^{e l}\right\}$ is diagonal, which immediately yields $J_{\rho_{0}, Q_{\alpha}^{e l}}^{c}=0$.

5.1.2. Photon induced electron current. It is hopeless to analyze the properties of (4.12) if we make no assumptions concerning $\rho^{e l}$ and the scattering operator $s_{c}$. The simplest assumptions is that $\rho^{e l}$ and $s_{c}$ commute. In this case, we get $\hat{\rho}^{e l}(\lambda)=\rho^{e l}(\lambda), \lambda \in \mathbb{R}$.

Lemma 5.4. Let $\mathcal{S}=\left\{H, H_{0}\right\}$ be the JCL-model. Furthermore, let $\rho^{\text {el }}$ be given by (5.2). If one of the cases $(E),(S)$ or $(C)$ of Proposition 5.3 is realized, then the $\rho^{e l}$ and $s_{c}$ commute.

Proof. If $(E)$ holds, then $\rho^{e l}=f\left(h_{0}^{e l}\right)$ which yields $\left[\rho^{e l}, s_{c}\right]=0$. If $(S)$ is valid, then the scattering matrix $\left\{s_{c}(\lambda)\right\}_{\lambda \in \mathbb{R}}$ is a scalar function which shows $\left[\rho^{e l}, s_{c}\right]=0$. Finally, if $(C)$ is realized, then the scattering matrix $\left\{s_{c}(\lambda)\right\}_{\lambda \in \mathbb{R}}$ diagonal. Since the $\rho^{e l}$ is given by (5.2) we get $\left[\rho^{e l}, s_{c}\right]=0$.

We will now calculate the current $J_{\rho_{0}, Q_{\alpha}^{\text {el }}}^{\text {, }}$, see (4.12). Clearly, we have $P_{\alpha}(\lambda)=$ $\sum_{n \in \mathbb{N}_{0}} p_{\alpha}^{e l}(\lambda-n \omega)$ and $I_{\mathfrak{h}(\lambda)}=P_{l}(\lambda)+P_{r}(\lambda), \lambda \in \mathbb{R}$. We then set:

$$
P_{n_{\alpha}}(\lambda):=P_{\alpha}(\lambda) P_{n}(\lambda)=P_{n}(\lambda) P_{\alpha}(\lambda)=p_{\alpha}^{e l}(\lambda-n \omega), \alpha \in\{l, r\},
$$

$n \in \mathbb{N}_{0}, \lambda \in \mathbb{R}$. In the following we use the notation $\widehat{T}_{p h}(\lambda)=\widehat{S}_{p h}(\lambda)-I_{\mathfrak{h}(\lambda)}, \lambda \in \mathbb{R}$, where $\left\{\widehat{T}_{p h}(\lambda)\right\}_{\lambda \in \mathbb{R}}$ is called the transition matrix and $\left\{\widehat{S}_{p h}(\lambda)\right\}_{\lambda \in \mathbb{R}}$ is given by (3.28). We set:

$$
\widehat{T}_{k_{\alpha} m_{\varkappa}}^{p h}(\lambda):=P_{k_{\alpha}}(\lambda) \widehat{T}_{p h}(\lambda) P_{m_{\varkappa}}(\lambda), \quad \lambda \in \mathbb{R}, \quad \alpha, \varkappa \in\{l, r\}, \quad k, m \in \mathbb{N}_{0},
$$

and:

$$
\widehat{\sigma}_{k_{\alpha} m_{\varkappa}}^{p h}(\lambda)=\operatorname{tr}\left(\widehat{T}_{k_{\alpha} m_{\varkappa}}^{p h}(\lambda)^{*} \widehat{T}_{k_{\alpha} m_{\varkappa}}^{p h}(\lambda)\right), \quad \lambda \in \mathbb{R},
$$

which is the cross-section between the channels $k_{\alpha}$ and $m_{\varkappa}$.

Proposition 5.5. Let $\mathcal{S}=\left\{H, H_{0}\right\}$ be the JCL-model.

(i) If $\rho^{\text {el }}$ commutes with the scattering operator $s_{c}$ and $q^{e l}$, then:

$$
J_{\rho_{0}, Q_{\alpha}^{e l}}^{p h}=-\sum_{\substack{m, n \in \mathbb{N}_{0} \\ \varkappa \in\{l, r\}}} \frac{\mathfrak{e}}{2 \pi} \int_{\mathbb{R}}\left(\rho^{p h}(n) f\left(\lambda-\mu_{\alpha}-n \omega\right)-\rho^{p h}(m) f\left(\lambda-\mu_{\varkappa}-m \omega\right)\right) \widehat{\sigma}_{n_{\alpha} m_{\varkappa}}^{p h}(\lambda) d \lambda .
$$

(ii) If in addition $\mathcal{S}=\left\{H, H_{0}\right\}$ is time reversible symmetric, then

$$
J_{\rho_{0}, Q_{\alpha}^{e l}}^{p h}=-\sum_{m, n \in \mathbb{N}_{0}} \frac{\mathfrak{e}}{2 \pi} \int_{\mathbb{R}}\left(\rho^{p h}(n) f\left(\lambda-\mu_{\alpha}-n \omega\right)-\rho^{p h}(m) f\left(\lambda-\mu_{\alpha^{\prime}}-m \omega\right)\right) \widehat{\sigma}_{n_{\alpha} m_{\alpha^{\prime}}}^{p h}(\lambda) d \lambda,
$$

$\alpha, \alpha^{\prime} \in\{l, r\}, \alpha \neq \alpha^{\prime}$. 
Proof. (i) Let us assume that:

$$
q^{e l}=\sum_{\varkappa \in\{l, r\}} g_{\varkappa}\left(h_{\varkappa}^{e l}\right)
$$

Notice that

$$
q_{a c}^{e l}(\lambda)=\sum_{\varkappa \in\{l, r\}} g_{\varkappa}(\lambda) p_{\varkappa}^{e l}(\lambda), \quad \lambda \in \mathbb{R} .
$$

Inserting (5.8) into (4.12) and using $q^{p h}=I_{\mathfrak{h} p h}$, we obtain the following:

$$
\begin{aligned}
J_{\rho_{0}, Q}^{p h}= & \sum_{\substack{m \in \mathbb{N}_{0} \\
\alpha \in\{l, r\}}} \rho^{p h}(m) \sum_{\substack{n \in \mathbb{N}_{0} \\
\varkappa \in\{l, r\}}} \frac{1}{2 \pi} \int_{\mathbb{R}} d \lambda \phi_{\alpha}(\lambda-m \omega) g_{\varkappa}(\lambda-n \omega) \times \\
& \operatorname{tr}\left(p_{\alpha}^{e l}(\lambda-m \omega)\left(p_{\varkappa}^{e l}(\lambda-n \omega) \delta_{m n}-\widehat{S}_{n m}^{p h}(\lambda)^{*} p_{\varkappa}^{e l}(\lambda-n \omega) \widehat{S}_{n m}^{p h}(\lambda)\right)\right),
\end{aligned}
$$

where, for simplicity, we have set:

$$
\phi_{\alpha}(\lambda):=f\left(\lambda-\mu_{\alpha}\right), \quad \lambda \in \mathbb{R}, \quad n \in \mathbb{N}_{0}, \quad \alpha \in\{l, r\} .
$$

Accordingly, we get:

$$
\begin{aligned}
J_{\rho_{0}, Q}^{p h}= & \sum_{\substack{n \in \mathbb{N}_{0} \\
\varkappa \in\{l, r\}}} \rho^{p h}(n) \frac{1}{2 \pi} \int_{\mathbb{R}} d \lambda \phi_{\varkappa}(\lambda-n \omega) g_{\varkappa}(\lambda-n \omega) \operatorname{tr}\left(p_{\varkappa}^{e l}(\lambda-n \omega)\right)- \\
& \sum_{\substack{n \in \mathbb{N}_{0} \\
\varkappa \in\{l, r\}}} \sum_{\substack{m \in \mathbb{N}_{0} \\
\alpha \in\{l, r\}}} \rho^{p h}(m) \frac{1}{2 \pi} \int_{\mathbb{R}} d \lambda \phi_{\alpha}(\lambda-m \omega) g_{\varkappa}(\lambda-n \omega) \times \\
& \operatorname{tr}\left(p_{\alpha}^{e l}(\lambda-m \omega) \widehat{S}_{n m}^{p h}(\lambda)^{*} p_{\varkappa}^{e l}(\lambda-n \omega) \widehat{S}_{n m}^{p h}(\lambda) p_{\alpha}^{e l}(\lambda-m \omega)\right) .
\end{aligned}
$$

Since the scattering matrix $\left\{\widehat{S}^{p h}(\lambda)\right\}_{\lambda \in \mathbb{R}}$ is unitary, we have:

$$
p_{\varkappa}^{e l}(\lambda-n \omega)=\sum_{\substack{m \in \mathbb{N}_{0} \\ \alpha \in\{l, r\}}} p_{\varkappa}^{e l}(\lambda-n \omega) \widehat{S}_{m n}^{p h}(\lambda)^{*} p_{\alpha}^{e l}(\lambda-m \omega) \widehat{S}_{m n}^{p h}(\lambda) p_{\varkappa}^{e l}(\lambda-n \omega),
$$

for $n \in \mathbb{N}_{0}$ and $\varkappa \in\{l, r\}$. Inserting (5.11) into (5.10), we find that:

$$
\begin{aligned}
J_{\rho_{0}, Q}^{p h}= & \sum_{\substack{n \in \mathbb{N}_{0} \\
\varkappa \in\{l, r\}}} \sum_{\substack{m \in \mathbb{N}_{0} \\
\alpha \in\{l, r\}}} \rho^{p h}(n) \frac{1}{2 \pi} \int_{\mathbb{R}} d \lambda \phi_{\varkappa}(\lambda-n \omega) g_{\varkappa}(\lambda-n \omega) \times \\
& \operatorname{tr}\left(p_{\varkappa}^{e l}(\lambda-n \omega) \widehat{S}_{n m}^{p h}(\lambda)^{*} p_{\alpha}^{e l}(\lambda-m \omega) \widehat{S}_{m n}^{p h}(\lambda) p_{\varkappa}^{e l}(\lambda-n \omega)\right)- \\
& \sum_{\substack{n \in \mathbb{N}_{0} \\
\varkappa \in\{l, r\}}} \sum_{\substack{m \in \mathbb{N}_{0} \\
\alpha \in\{l, r\}}} \rho^{p h}(m) \frac{1}{2 \pi} \int_{\mathbb{R}} d \lambda \phi_{\alpha}(\lambda-m \omega) g_{\varkappa}(\lambda-n \omega) \times \\
& \operatorname{tr}\left(p_{\alpha}^{e l}(\lambda-m \omega) \widehat{S}_{n m}^{p h}(\lambda)^{*} p_{\varkappa}^{e l}(\lambda-n \omega) \widehat{S}_{n m}^{p h}(\lambda) p_{\alpha}^{e l}(\lambda-m \omega)\right) .
\end{aligned}
$$

Using the notation (5.5), we find the following:

$$
\begin{aligned}
J_{\rho_{0}, Q}^{p h}= & \sum_{\substack{n \in \mathbb{N}_{0} \\
\varkappa \in\{l, r\}}} \sum_{\substack{m \in \mathbb{N}_{0} \\
\alpha \in\{l, r\}}} \rho^{p h}(n) \frac{1}{2 \pi} \int_{\mathbb{R}} d \lambda \phi_{\varkappa}(\lambda-n \omega) g_{\varkappa}(\lambda-n \omega) \hat{\sigma}_{m_{\alpha} n_{\varkappa}}^{p h}(\lambda)- \\
& \sum_{\substack{n \in \mathbb{N}_{0} \\
\varkappa \in\{l, r\}}} \sum_{\substack{m \in \mathbb{N}_{0} \\
\alpha \in\{l, r\}}} \rho^{p h}(m) \frac{1}{2 \pi} \int_{\mathbb{R}} d \lambda \phi_{\alpha}(\lambda-m \omega) g_{\varkappa}(\lambda-n \omega) \hat{\sigma}_{n_{\varkappa} m_{\alpha}}^{p h}(\lambda) .
\end{aligned}
$$


By (3.16), we find that:

$$
\sum_{\substack{m \in \mathbb{N}_{0} \\ \alpha \in\{l, r\}}} \widehat{\sigma}_{m_{\alpha} n_{\varkappa}}^{p h}(\lambda)=\sum_{\substack{m \in \mathbb{N}_{0} \\ \alpha \in\{l, r\}}} \widehat{\sigma}_{n_{\varkappa} m_{\alpha}}^{p h}(\lambda) \quad \lambda \in \mathbb{R} .
$$

Using that, we obtain the following:

$$
\begin{aligned}
& J_{\rho_{0}, Q}^{p h}=\sum_{\substack{m, n \in \mathbb{N}_{0} \\
\alpha, \varkappa \in\{l, r\}}} \frac{1}{2 \pi} \int_{\mathbb{R}} \times \\
& \left(\rho^{p h}(n) \phi_{\varkappa}(\lambda-n \omega)-\rho^{p h}(m) \phi_{\alpha}(\lambda-m \omega)\right) g_{\varkappa}(\lambda-n \omega) \widehat{\sigma}_{n_{\varkappa} m_{\alpha}}^{p h}(\lambda) d \lambda .
\end{aligned}
$$

Setting $g_{\alpha}(\lambda)=-\mathfrak{e}$ and $g_{\varkappa}(\lambda) \equiv 0, \varkappa \neq \alpha$, we obtain (5.6).

(ii) Straightforward computation shows that:

$$
\begin{gathered}
\sum_{n, m \in \mathbb{N}_{0}} \int_{\mathbb{R}}\left(\rho^{p h}(n) f\left(\lambda-\mu_{a}-n \omega\right)-\rho^{p h}(m) f\left(\lambda-\mu_{a}-m \omega\right)\right) \widehat{\sigma}_{n_{\alpha} m_{\alpha}}^{p h}(\lambda) d \lambda= \\
\sum_{n, m \in \mathbb{N}_{0}} \int_{\mathbb{R}}\left(\rho^{p h}(m) f\left(\lambda-\mu_{a}-m \omega\right)-\rho^{p h}(n) f\left(\lambda-\mu_{a}-n \omega\right)\right) \widehat{\sigma}_{m_{\alpha} n_{\alpha}}^{p h}(\lambda) d \lambda .
\end{gathered}
$$

Since $\sigma_{m_{\alpha} n_{\alpha}}^{p h}(\lambda)=\sigma_{n_{\alpha} m_{\alpha}}^{p h}(\lambda), \lambda \in \mathbb{R}$, we obtain the following:

$$
\begin{aligned}
& \sum_{n, m \in \mathbb{N}_{0}} \int_{\mathbb{R}}\left(\rho^{p h}(n) f\left(\lambda-\mu_{a}-n \omega\right)-\rho^{p h}(m) f\left(\lambda-\mu_{a}-m \omega\right)\right) \widehat{\sigma}_{n_{\alpha} m_{\alpha}}^{p h}(\lambda) d \lambda= \\
& -\sum_{n, m \in \mathbb{N}_{0}} \int_{\mathbb{R}}\left(\rho^{p h}(n) f\left(\lambda-\mu_{a}-n \omega\right)-\rho^{p h}(m) f\left(\lambda-\mu_{a}-m \omega\right)\right) \widehat{\sigma}_{n_{\alpha} m_{\alpha}}^{p h}(\lambda) d \lambda
\end{aligned}
$$

which yields:

$$
\sum_{n, m \in \mathbb{N}_{0}} \int_{\mathbb{R}}\left(\rho^{p h}(n) f\left(\lambda-\mu_{a}-n \omega\right)-\rho^{p h}(m) f\left(\lambda-\mu_{a}-m \omega\right)\right) \widehat{\sigma}_{n_{\alpha} m_{\alpha}}^{p h}(\lambda) d \lambda=0 .
$$

Using that, we immediately obtain the representation (5.7) from (5.6).

Corollary 5.6. Let $\mathcal{S}=\left\{H, H_{0}\right\}$ be the JCL-model.

(i) If the cases cases $(E),(S)$ or $(C)$ of Proposition 5.3 are realized, then the representation (5.6) holds.

(ii) If the case (E) of Proposition 5.3 is realized and the system $\mathcal{S}=\left\{H, H_{0}\right\}$ is time reversible symmetric, then

$$
J_{\rho_{0}, Q_{\alpha}^{e l}}^{p h}=-\sum_{m, n \in \mathbb{N}_{0}} \frac{\mathfrak{e}}{2 \pi} \int_{\mathbb{R}}\left(\rho^{p h}(n) f(\lambda-\mu-n \omega)-\rho^{p h}(m) f(\lambda-\mu-m \omega)\right) \widehat{\sigma}_{n_{\alpha} m_{\alpha^{\prime}}}^{p h}(\lambda) d \lambda
$$

$n \in \mathbb{N}_{0}, \alpha \in\{l, r\}$ where $\mu:=\mu_{l}=\mu_{r}$ and $\alpha \neq \alpha^{\prime}$.

(iii) If the case (E) of Proposition 5.3 is realized and the system $\mathcal{S}=\left\{H, H_{0}\right\}$ is time reversible and mirror symmetric, then $J_{\rho_{0}, Q_{\alpha}^{e l}}^{p h}=0$.

Proof. (i) The statement follows from Proposition 5.5(i) and Lemma 5.4.

(ii) Setting $\mu_{\alpha}=\mu_{\alpha^{\prime}}$ formula (5.13) follows (5.7).

(iii) If $\mathcal{S}=\left\{H, H_{0}\right\}$ is time reversible and mirror symmetric, we obtain from Lemma 2.14 (ii) that $\widehat{\sigma}_{n_{\alpha} m_{\alpha^{\prime}}}^{p h}(\lambda)=\widehat{\sigma}_{n_{\alpha^{\prime}} m_{\alpha}}^{p h}(\lambda), \lambda \in \mathbb{R}, n, m \in \mathbb{N}_{0}, \alpha, \alpha^{\prime} \in\{l, r\}, \alpha \neq \alpha^{\prime}$. Using that, we obtain from (5.13) the following:

$$
J_{\rho_{0}, Q_{\alpha}^{e l}}^{p h}=-\sum_{m, n \in \mathbb{N}_{0}} \frac{\mathfrak{e}}{2 \pi} \int_{\mathbb{R}}\left(\rho^{p h}(n) f(\lambda-\mu-n \omega)-\rho^{p h}(m) f(\lambda-\mu-m \omega)\right) \hat{\sigma}_{n_{\alpha^{\prime}} m_{\alpha}}^{p h}(\lambda) d \lambda .
$$


Interchanging $m$ and $n$, we obtain:

$$
J_{\rho_{0}, Q_{\alpha}^{e l}}^{p h}=-\sum_{m, n \in \mathbb{N}_{0}} \frac{\mathfrak{e}}{2 \pi} \int_{\mathbb{R}}\left(\rho^{p h}(m) f(\lambda-\mu-m \omega)-\rho^{p h}(n) f(\lambda-\mu-n \omega)\right) \widehat{\sigma}_{m_{\alpha^{\prime}} n_{\alpha}}^{p h}(\lambda) d \lambda .
$$

Using that $S$ is time reversible symmetric we get from Lemma 2.14 (i) that:

$$
J_{\rho_{0}, Q_{\alpha}^{e l}}^{p h}=-\sum_{m, n \in \mathbb{N}_{0}} \frac{\mathfrak{e}}{2 \pi} \int_{\mathbb{R}}\left(\rho^{p h}(m) f(\lambda-\mu-m \omega)-\rho^{p h}(n) f(\lambda-\mu-n \omega)\right) \hat{\sigma}_{n_{\alpha} m_{\alpha^{\prime}}}^{p h}(\lambda) d \lambda .
$$

which shows that $J_{\rho_{0}, Q_{\alpha}^{e l}}^{p h}=-J_{\rho_{0}, Q_{\alpha}^{e l}}^{p h}$. Hence $J_{\rho_{0}, Q_{\alpha}^{e l}}^{p h}=0$.

We note that by Proposition 5.3, the contact induced current is zero, i.e. $J_{\rho_{0}, Q_{\alpha}^{e l}}^{c}=0$. Hence, if the $\mathcal{S}$ is time reversible and mirror symmetric, then the total current is zero, i.e. $J_{\rho_{0}, Q_{\alpha}^{e l}}^{\mathcal{S}}=0$.

Remark 5.7. Let the case $(E)$ of Proposition 5.3 be realized, that is, $\mu_{l}=\mu_{r}$. Moreover, we assume for simplicity that $0=: v_{r} \leqslant v:=v_{l}$.

(i) If $\beta=\infty$, then $\rho^{p h}(n)=\delta_{0 n}, n \in \mathbb{N}_{0}$. From (5.6), we immediately obtain that $J_{\rho^{e l}, Q_{\alpha}^{e l}}^{p h}=0$. That means, if the temperature is zero, then the photon-induced electron current is zero.

(ii) The photon-induced electron current might be zero even if $\beta<\infty$. Indeed, let $\mathcal{S}=$ $\left\{H, H_{0}\right\}$ be time reversible symmetric and let the case (E) be realized. If $\omega \geqslant v+4$ and $\mathfrak{h}^{e l}(\lambda):=\mathfrak{h}_{n}^{e l}(\lambda)=\mathfrak{h}^{e l}(\lambda-n \omega), n \in \mathbb{N}_{0}$. Hence, one always has $n=m$ in formula (5.13), which immediately yields $J_{\rho_{0}, Q_{\alpha}^{e l}}^{p h}=0$.

(iii) The photon-induced electron current might be different than zero. Indeed, let $\mathcal{S}=\left\{H, H_{0}\right\}$ be time reversible symmetric and let $v=2$ and $\omega=4$, then one sees that to calculate the $J_{\rho_{0}, Q_{l}^{e l}}^{p h}$, one has to consider $m=n+1$ in formula (5.13). Therefore, we find that:

$$
\begin{aligned}
& J_{\rho_{0}, Q_{l}^{e l}}^{p h}=-\sum_{n \in \mathbb{N}_{0}} \frac{\mathfrak{e}}{2 \pi} \times \\
& \int_{\mathbb{R}} d \lambda\left(\rho^{p h}(n) f(\lambda-\mu-n \omega)-\rho^{p h}(n+1) f(\lambda-\mu-(n+1) \omega)\right) \widehat{\sigma}_{n_{l}(n+1)_{r}}^{p h}(\lambda) .
\end{aligned}
$$

If $\rho^{p h}$ is given by (5.3) and $f(\lambda)=f_{F D}(\lambda)$, cf. (3.21), then one easily verifies that

$$
\frac{\partial}{\partial x} \rho^{p h}(x) f_{F D}(\lambda-\mu-x \omega)<0, \quad x, \mu, \lambda \in \mathbb{R} .
$$

Hence, $\rho^{p h}(n) f_{F D}(\lambda-\mu-n \omega)$ is decreasing in $n \in \mathbb{N}_{0}$ for $\lambda, \mu \in \mathbb{R}$ which yields $\left(\rho^{p h}(n) f(\lambda-\mu-n \omega)-\rho^{p h}(n+1) f(\lambda-\mu-(n+1) \omega)\right) \geqslant 0$. Therefore, $J_{\rho_{0}, Q_{l}^{e l}}^{p h} \leqslant 0$ which means that the photon-induced current leaves the left-hand side and enters the righthand side. In fact, $J_{\rho_{0}, Q_{l}^{e l}}^{p h}=0$ implies that $\widehat{\sigma}_{n_{l}(n+1)_{r}}^{p h}(\lambda)=0$ for $n \in \mathbb{N}_{0}$ and $\lambda \in \mathbb{R}$, which means that there is no scattering from the left-hand side to the right one and vice versa which can be excluded generically.

\subsection{Photon current}

The photon current is related to the charge by equation:

$$
Q:=Q^{p h}=-I_{\mathfrak{h}}{ }^{e l} \otimes \mathfrak{n},
$$

where $\mathfrak{n}=\mathrm{d} \Gamma(1)=b^{*} b$ is the photon number operator on $\mathfrak{h}^{p h}=\mathfrak{F}_{+}(\mathbb{C})$, which is self-adjoint and commutes with $h^{p h}$. It follows that $Q^{p h}$ is also self-adjoint and commutes with $H_{0}$. It is not bounded, but since $\operatorname{dom}(\mathfrak{n})=\operatorname{dom}\left(h^{p h}\right)$, it is immediately obvious that $Q^{p h}\left(H_{0}+\theta\right)^{-1}$ is bounded, 
when $\mathfrak{N}$ is a tempered charge. Its charge matrix with respect to the spectral representation $\Pi\left(H_{0}^{a c}\right)$ of Lemma 2.12 is given by:

$$
Q_{a c}^{p h}(\lambda)=-\bigoplus_{n \in \mathbb{N}_{0}} n P_{n}(\lambda)
$$

We recall that $P_{n}(\lambda)$ is the orthogonal projection from $\mathfrak{h}(\lambda)$ onto $\mathfrak{h}_{n}(\lambda)=\mathfrak{h}^{\text {el }}(\lambda-n \omega), \lambda \in \mathbb{R}$. We will now calculate the photon current or, as it is also known, the photon production rate.

5.2.1. Contact induced photon current. The following proposition is, in fact, in accordance with the physical intuition.

Proposition 5.8. Let $\mathcal{S}=\left\{H, H_{0}\right\}$ be the JCL-model. Then $J_{\rho_{0}, Q^{p h}}^{c}=0$.

Proof. We note that $q_{a c}^{e l}(\lambda)=I_{\mathfrak{h}}^{e l}(\lambda), \lambda \in \mathbb{R}$. Inserting this into (3.25), we obtain $J_{\rho^{e l}, q^{e l}}^{s_{c}}=0$. Applying Proposition 4.2 we prove $J_{\rho_{0}, Q^{p h}}^{c}=0$.

The result reflects the fact that the lead contact does not contribute to the photon current, which is plausible from the physical point of view.

5.2.2. Photon current. From Proposition 5.8, we see that only the photon-induced photon current $J_{\rho_{0}, Q^{p h}}^{p h}$ contributes to the photon current $J_{\rho_{0}, Q^{p h}}^{\mathcal{S}}$. Since $J_{\rho_{0}, Q^{p h}}^{\mathcal{S}}=J_{\rho_{0}, Q^{p h}}^{p h}$, we call $J_{\rho_{0}, Q^{p h}}^{p h}$ simply the photon current.

Using the notation $\widehat{T}_{n m}^{p h}(\lambda):=P_{n}(\lambda) \widehat{T}_{p h}(\lambda) \uparrow \mathfrak{h}^{e l}(\lambda-m \omega), \lambda \in \mathbb{R}, m, n \in \mathbb{N}_{0}$. We set:

$$
\widetilde{T}_{n m}^{p h}(\lambda)=\widehat{T}_{n m}^{p h}(\lambda) s_{c}(\lambda-m \omega), \quad \lambda \in \mathbb{R}, \quad m, n \in \mathbb{N}_{0},
$$

and

$$
\widetilde{T}_{n_{\varkappa} m_{\alpha}}^{p h}(\lambda):=P_{n_{\varkappa}}(\lambda) \widetilde{T}_{n m}^{p h}(\lambda)\left\lceil\mathfrak{h}_{\alpha}^{e l}(\lambda-m \omega), \quad \lambda \in \mathbb{R},\right.
$$

$m, n \in \mathbb{N}_{0}, \alpha, \varkappa \in\{l, r\}$, as well as $\widetilde{\sigma}_{n_{\varkappa} m_{\alpha}}^{p h}(\lambda):=\operatorname{tr}\left(\widetilde{T}_{n_{\varkappa} m_{\alpha}}^{p h}(\lambda)^{*} \widetilde{T}_{n_{\varkappa} m_{\alpha}}^{p h}(\lambda)\right), \lambda \in \mathbb{R}$.

Proposition 5.9. Let $\mathcal{S}=\left\{H, H_{0}\right\}$ be the JCL-model.

(i) Then:

$$
J_{\rho_{0}, Q^{p h}}^{p h}=\sum_{\substack{m, n \in \mathbb{N}_{0} \\ \alpha, \varkappa \in\{l, r\}}}(n-m) \rho^{p h}(m) \frac{1}{2 \pi} \int_{\mathbb{R}} f\left(\lambda-\mu_{\alpha}-m \omega\right) \widetilde{\sigma}_{n_{\varkappa} m_{\alpha}}^{p h}(\lambda) d \lambda .
$$

(ii) If $\rho^{e l}$ commutes with $s_{c}$, then:

$$
J_{\rho_{0}, Q^{p h}}^{p h}=\sum_{\substack{m, n \in \mathbb{N}_{0} \\ \alpha, \varkappa \in\{l, r\}}}(n-m) \rho^{p h}(m) \frac{1}{2 \pi} \int_{\mathbb{R}} f\left(\lambda-\mu_{\alpha}-m \omega\right) \widehat{\sigma}_{n_{\varkappa} m_{\alpha}}^{p h}(\lambda) d \lambda .
$$

(iii) If $\rho^{e l}$ commutes with $s_{c}$ and $\mathcal{S}=\left\{H, H_{0}\right\}$ is time reversible symmetric, then:

$$
\begin{aligned}
& J_{\rho_{0}, Q^{p h}}^{p h}=\sum_{\substack{m, n \in \mathbb{N}_{0}, n>m \\
\varkappa, \alpha \in\{l, r\}}} \frac{1}{2 \pi} \int_{\mathbb{R}} d \lambda \times \\
& (n-m)\left(\rho^{p h}(m) f\left(\lambda-\mu_{\alpha}-m \omega\right)-\rho^{p h}(n) f\left(\lambda-\mu_{\varkappa}-n \omega\right)\right) \widehat{\sigma}_{n_{\varkappa} m_{\alpha}}^{p h}(\lambda),
\end{aligned}
$$

where $\alpha^{\prime} \in\{l, r\}$ and $\alpha^{\prime} \neq \alpha$. 
Proof. (i) From (4.12) we get

$$
\begin{aligned}
J_{\rho_{0}, Q^{p h}}^{p h} & =-\sum_{m, n \in \mathbb{N}_{0}} n \rho^{p h}(m) \times \\
& \frac{1}{2 \pi} \int_{\mathbb{R}} d \lambda \operatorname{tr}\left(\widehat{\rho}_{a c}^{e l}(\lambda-m \omega)\left(P_{n}(\lambda) \delta_{m n}-\widehat{S}_{n m}^{p h}(\lambda)^{*} q_{a c}^{e l}(\lambda-n \omega) \widehat{S}_{n m}^{p h}(\lambda)\right)\right) .
\end{aligned}
$$

Hence:

$$
\begin{aligned}
& J_{\rho_{0}, Q^{p h}}^{p h}=-\sum_{m \in \mathbb{N}_{0}} m \rho^{p h}(m) \frac{1}{2 \pi} \int_{\mathbb{R}} \operatorname{tr}\left(\widehat{\rho}_{a c}^{e l}(\lambda-m \omega)\left(P_{m}(\lambda)-\widehat{S}_{m m}^{p h}(\lambda)^{*} P_{m}(\lambda) \widehat{S}_{m m}^{p h}(\lambda)\right)\right) d \lambda+ \\
& \sum_{\substack{m, n \in \mathbb{N}_{0} \\
m \neq n}} n \rho^{p h}(m) \frac{1}{2 \pi} \int_{\mathbb{R}} \operatorname{tr}\left(\widehat{\rho}_{a c}^{e l}(\lambda-m \omega) \widehat{S}_{n m}^{p h}(\lambda)^{*} P_{n}(\lambda) \widehat{S}_{n m}^{p h}(\lambda)\right) d \lambda .
\end{aligned}
$$

Using the relation $P_{m}(\lambda)=I_{\mathfrak{h}(\lambda)}-\sum_{n \in \mathbb{N}_{0}, m \neq n} P_{n}(\lambda), \lambda \in \mathbb{R}$, we obtain the following:

$$
\begin{aligned}
J_{\rho_{0}, Q^{p h}}^{p h} & =-\sum_{\substack{m, n \in \mathbb{N}_{0} \\
m \neq n}} m \rho^{p h}(m) \frac{1}{2 \pi} \int_{\mathbb{R}} \operatorname{tr}\left(\widehat{\rho}_{a c}^{e l}(\lambda-m \omega)\left(\widehat{S}_{n m}^{p h}(\lambda)^{*} P_{n}(\lambda) \widehat{S}_{n m}^{p h}(\lambda)\right)\right) d \lambda+ \\
& \sum_{\substack{m, n \in \mathbb{N}_{0} \\
m \neq n}} n \rho^{p h}(m) \frac{1}{2 \pi} \int_{\mathbb{R}} \operatorname{tr}\left(\widehat{\rho}_{a c}^{e l}(\lambda-m \omega) \widehat{S}_{n m}^{p h}(\lambda)^{*} P_{n}(\lambda) \widehat{S}_{n m}^{p h}(\lambda)\right) d \lambda .
\end{aligned}
$$

Since $\widehat{T}_{p h}(\lambda)=\widehat{S}_{p h}(\lambda)-I_{\mathfrak{h}(\lambda)}, \lambda \in \mathbb{R}$, we find

$$
J_{\rho_{0}, Q^{p h}}^{p h}=-\sum_{m, n \in \mathbb{N}_{0}}(m-n) \rho^{p h}(m) \frac{1}{2 \pi} \int_{\mathbb{R}} \operatorname{tr}\left(\widehat{\rho}_{a c}^{e l}(\lambda-m \omega) \widehat{T}_{n m}^{p h}(\lambda)^{*} \widehat{T}_{n m}^{p h}(\lambda)\right) d \lambda .
$$

Using (4.13) and definition (5.14) one readily sees that:

$$
J_{\rho_{0}, Q^{p h}}^{p h}=-\sum_{m, n \in \mathbb{N}_{0}}(m-n) \rho^{p h}(m) \frac{1}{2 \pi} \int_{\mathbb{R}} \operatorname{tr}\left(\rho_{a c}^{e l}(\lambda-m \omega) \widetilde{T}_{n m}^{p h}(\lambda)^{*} \widetilde{T}_{n m}^{p h}(\lambda)\right) d \lambda .
$$

Since $\rho_{a c}^{e l}=\rho_{l}^{e l} \oplus \rho_{r}^{e l}$ where $\rho_{\alpha}^{e l}$ is given by (5.2), we find the following:

$$
J_{\rho_{0}, Q^{p h}}^{p h}=-\sum_{\substack{m, n \in \mathbb{N}_{0} \\ \alpha, \varkappa \in\{l, r\}}}(m-n) \rho^{p h}(m) \frac{1}{2 \pi} \int_{\mathbb{R}} f\left(\lambda-\mu_{\alpha}-m \omega\right) \operatorname{tr}\left(\widetilde{T}_{n_{\varkappa} m_{\alpha}}^{p h}(\lambda)^{*} \widetilde{T}_{n_{\varkappa} m_{\alpha}}^{p h}(\lambda)\right) d \lambda,
$$

where we have used (5.15). Using $\widetilde{\sigma}_{n_{\varkappa} m_{\alpha}}^{p h}(\lambda)=\operatorname{tr}\left(\widetilde{T}_{n_{\varkappa} m_{\alpha}}^{p h}(\lambda)^{*} \widetilde{T}_{n_{\varkappa} m_{\alpha}}^{p h}(\lambda)\right)$, we prove (5.16).

(ii) If $\rho_{a c}^{e l}$ commutes with $s_{c}$, then $\hat{\rho}_{a c}^{e l}(\lambda)=\rho_{a c}^{e l}(\lambda), \lambda \in \mathbb{R}$, which yields that one can replace $\widetilde{\sigma}_{n_{\varkappa} m_{\alpha}}^{p h}(\lambda)$ by $\widehat{\sigma}_{n_{\varkappa} m_{\alpha}}^{p h}(\lambda), \lambda \in \mathbb{R}$. Therefore, (5.17) holds.

(iii) Clearly, we have:

$$
\begin{aligned}
& J_{\rho_{0}, Q^{p h}}^{p h}= \\
& \quad \sum_{\substack{m, n \in \mathbb{N}_{0}, n>m \\
\alpha, \varkappa \in\{l, r\}}}(n-m) \rho^{p h}(m) \frac{1}{2 \pi} \int_{\mathbb{R}} f\left(\lambda-\mu_{\alpha}-m \omega\right) \hat{\sigma}_{n_{\varkappa} m_{\alpha}}^{p h}(\lambda) d \lambda+ \\
& \sum_{\substack{m, n \in \mathbb{N}_{0}, n<m \\
\alpha, \varkappa \in\{l, r\}}}(n-m) \rho^{p h}(m) \frac{1}{2 \pi} \int_{\mathbb{R}} f\left(\lambda-\mu_{\alpha}-m \omega\right) \widehat{\sigma}_{n_{\varkappa} m_{\alpha}}^{p h}(\lambda) d \lambda .
\end{aligned}
$$


Moreover, a straightforward computation shows the following:

$$
\begin{gathered}
\sum_{\substack{m, n \in \mathbb{N}_{0}, n<m \\
\alpha, \varkappa \in\{l, r\}}}(n-m) \rho^{p h}(m) \frac{1}{2 \pi} \int_{\mathbb{R}} f\left(\lambda-\mu_{\alpha}-m \omega\right) \widehat{\sigma}_{n_{\varkappa} m_{\alpha}}^{p h}(\lambda) d \lambda= \\
\sum_{\substack{m, n \in \mathbb{N}_{0}, n>m \\
\alpha, \varkappa \in\{l, r\}}}(m-n) \rho^{p h}(n) \frac{1}{2 \pi} \int_{\mathbb{R}} f\left(\lambda-\mu_{\varkappa}-n \omega\right) \widehat{\sigma}_{m_{\alpha} n_{\varkappa}}^{p h}(\lambda) d \lambda .
\end{gathered}
$$

Since $\mathcal{S}=\left\{H, H_{0}\right\}$ is time reversible symmetric, we find the following:

$$
\begin{gathered}
\sum_{\substack{m, n \in \mathbb{N}_{0}, n<m \\
\alpha, \varkappa \in\{l, r\}}}(n-m) \rho^{p h}(m) \frac{1}{2 \pi} \int_{\mathbb{R}} f\left(\lambda-\mu_{\alpha}-m \omega\right) \widehat{\sigma}_{m_{\alpha}}^{p h}(\lambda) d \lambda= \\
\sum_{\substack{m, n \in \mathbb{N}_{0}, n>m \\
\alpha, \varkappa \in\{l, r\}}}(m-n) \rho^{p h}(n) \frac{1}{2 \pi} \int_{\mathbb{R}} f\left(\lambda-\mu_{\varkappa}-n \omega\right) \widehat{\sigma}_{n_{\varkappa} m_{\alpha}}^{p h}(\lambda) d \lambda .
\end{gathered}
$$

Inserting (5.20) into (5.19), we obtain (5.18).

Corollary 5.10. Let $\mathcal{S}=\left\{H, H_{0}\right\}$ be the JCL-model and let $f=f_{F D}$. If case (E) of Proposition 5.3 is realized and $\mathcal{S}=\left\{H, H_{0}\right\}$ is time reversible symmetric, then $J_{\rho_{0}, Q^{p h}}^{p h} \geqslant 0$.

Proof. We set $\mu:=\mu_{l}=\mu_{r}$. One has:

$$
\begin{aligned}
& \rho^{p h}(m) f(\lambda-\mu-m \omega)-\rho^{p h}(n) f(\lambda-\mu-n \omega)= \\
& \quad e^{-m \beta \omega}\left(1-e^{-(n-m) \beta \omega}\right) f_{F D}(\lambda-\mu-m \omega) f_{F D}(\lambda-\mu-n \omega) \geqslant 0,
\end{aligned}
$$

for $n>m$. From (5.18), we see that $J_{\rho_{0}, Q^{p h}}^{p h} \geqslant 0$.

Remark 5.11. We will now comment the results. If $J_{\rho_{0}, Q^{p h}}^{p h} \geqslant 0$, then system $S$ is called light emitting. Similarly, if $J_{\rho_{0}, Q^{p h}}^{p h} \leqslant 0$, then we call it light absorbing. Of course if $\mathcal{S}$ is light emitting and absorbing, then $J_{\rho_{0}, Q^{p h}}^{p h}=0$.

(i) If $\beta=\infty$, then $\rho^{p h}(m)=\delta_{0 m}, m \in \mathbb{N}_{0}$. Inserting this into (5.16), we get:

$$
J_{\rho_{0}, Q^{p h}}^{p h}=\sum_{\substack{n \in \mathbb{N}_{0} \\ \alpha, \varkappa \in\{l, r\}}} n \frac{1}{2 \pi} \int_{\mathbb{R}} f\left(\lambda-\mu_{\alpha}\right) \widetilde{\sigma}_{n_{\varkappa} 0_{\alpha}}^{p h}(\lambda) d \lambda \geqslant 0
$$

Hence, the system $\mathcal{S}$ is light emitting.

(ii) Let us show $\mathcal{S}$ might be light emitting even if $\beta<\infty$. We consider the case $(E)$ of Proposition 5.3. If $\mathcal{S}$ is time reversible symmetric, then it follows from Corollary 5.10 that the system is light emitting.

If the system $S$ is time reversible and mirror symmetric, then $J_{\rho_{0}, Q_{\alpha}^{e l}}^{p h}=0, \alpha \in\{l, r\}$, by Corollary 5.6(iii). Since $J_{\rho_{0}, Q^{e l}}^{c}=0$ by Proposition 5.3, we get that $J_{\rho_{0}, Q_{\alpha}^{e l}}^{\mathcal{S}}=0$ but the photon current is larger than zero. So our $J C L$-model is light emitting by a zero total electron current $J_{\rho_{0}, Q_{\alpha}^{e l}}^{\mathcal{S}}$.

Let $v_{r}=0, v_{l}=2$ and $\omega=4$. Hence $\mathcal{S}$ is not mirror symmetric. Then, we get from Remark 5.7(iii) that $J_{\rho_{0}, Q_{l}^{e l}}^{p h}=-J_{\rho_{0}, Q_{r}^{e l}}^{p h} \leqslant 0$. Hence, there is an electron current from the left to the right lead. Notice that by Proposition $5.3 J_{\rho_{0}, Q_{l}^{e l}}^{c}=0$. Hence, $J_{\rho_{0}, Q_{l}^{e l}}^{\mathcal{S}} \leqslant 0$. 
(iii) To realize a light absorbing situation, we consider the case $(S)$ of Proposition 5.3 and assume that $S$ is time reversible symmetric. Notice that by Lemma 5.4, $s_{c}$ commutes with $\rho^{e l}$. We make the following choices:

$$
v_{r}=0, \quad v_{l} \geqslant 4, \quad \omega=v_{l}, \quad \mu_{l}=0, \quad \mu_{r}=\omega=v_{l} .
$$

It follows out that with respect to the representation (5.18) one has only to $m=n-1$, $\varkappa=r$ and $\alpha=l$. Hence,

$$
\begin{aligned}
J_{\rho_{0}, Q^{p h}}^{p h} & =\sum_{n \in \mathbb{N}} \frac{1}{2 \pi} \times \\
& \int_{\mathbb{R}} d \lambda\left(\rho^{p h}(n-1) f(\lambda-(n-1) \omega)-\rho^{p h}(n) f(\lambda-(n+1) \omega)\right) \widehat{\sigma}_{n_{l}(n-1)_{r}}^{p h}(\lambda)
\end{aligned}
$$

Since, $f(\lambda)=f_{F D}(\lambda)$, we find:

$$
\begin{gathered}
\rho^{p h}(n-1) f(\lambda-(n-1) \omega)-\rho^{p h}(n) f(\lambda-(n+1) \omega)= \\
\rho^{p h}(n-1) f(\lambda-(n-1) \omega) f(\lambda-(n+1) \omega) \times \\
\left(1+e^{\beta(\lambda-(n+1) \omega)}-e^{-\beta \omega}\left(1+e^{\beta(\lambda-\omega(n-1))}\right)\right),
\end{gathered}
$$

or

$$
\begin{aligned}
& \rho^{p h}(n-1) f(\lambda-(n-1) \omega)-\rho^{p h}(n) f(\lambda-(n+1) \omega)= \\
& \quad \rho^{p h}(n-1) f(\lambda-(n-1) \omega) f(\lambda-(n+1) \omega)\left(1-e^{-\beta \omega}\right)\left(1-e^{\beta(\lambda-\omega n)}\right) .
\end{aligned}
$$

Since $\lambda-n \omega \geqslant 0$ we find $\rho^{p h}(n-1) f(\lambda-(n-1) \omega)-\rho^{p h}(n) f(\lambda-(n+1) \omega) \leqslant 0$ which yields $J_{\rho_{0}, Q^{p h}}^{p h} \leqslant 0$.

To calculate $J_{\rho_{0}, Q_{l}^{e l}}^{p h}$, we use formula (5.7). Setting $\alpha=l$, we obtain $\alpha^{\prime}=r$, which yields

$$
\begin{aligned}
J_{\rho_{0}, Q_{l}^{e l}}^{p h}= & -\sum_{m, n \in \mathbb{N}_{0}} \frac{\mathfrak{e}}{2 \pi} \times \\
& \int_{\mathbb{R}} d \lambda\left(\rho^{p h}(n) f\left(\lambda-\mu_{r}-n \omega\right)-\rho^{p h}(m) f\left(\lambda-\mu_{l}-m \omega\right)\right) \widehat{\sigma}_{n_{l} m_{r}}^{p h}(\lambda) .
\end{aligned}
$$

One verifies that $\widehat{\sigma}_{0_{l} 0_{r}}^{p h}(\lambda)=0$ and $\widehat{\sigma}_{n_{l} m_{r}}^{p h}(\lambda)=0$ for $m \neq n+1, n \in \mathbb{N}$. Hence,

$$
\begin{aligned}
J_{\rho_{0}, Q_{l}^{e l}}^{p h} & =-\sum_{n \in \mathbb{N}} \frac{\mathfrak{e}}{2 \pi} \times \\
& \int_{\mathbb{R}} d \lambda\left(\rho^{p h}(n) f\left(\lambda-\mu_{r}-n \omega\right)-\rho^{p h}(n-1) f\left(\lambda-\mu_{l}-(n+1) \omega\right)\right) \hat{\sigma}_{n_{l}(n+1)_{r}}^{p h}(\lambda),
\end{aligned}
$$

Since $\mu_{r}=\omega$ and $\mu_{l}=0$, we find:

$$
\begin{aligned}
J_{\rho_{0}, Q_{l}^{e l}}^{p h}= & -\sum_{n \in \mathbb{N}} \frac{\mathfrak{e}}{2 \pi} \times \\
& \int_{\mathbb{R}} f(\lambda-(n+1) \omega) \rho^{p h}(n-1)\left(1-e^{-\beta \omega}\right) \widehat{\sigma}_{n_{l}(n+1)_{r}}^{p h}(\lambda) d \lambda \leqslant 0 .
\end{aligned}
$$

Hence, there is electron current flowing from the left to right induced by photons. We recall that $J_{\rho_{0}, Q_{l}^{e l}}^{c}=0$. 


\section{Acknowledgments}

The first two authors would like to thank the University of Aalborg and the Centre de Physique Théorique - Luminy for hospitality and financial support. We thank Horia D. Cornean for discussions concerning the $J C L$-model and Igor Yu.Popov for the opportunity to present this complete version of our results. The work on this paper has been partially supported by the ERC grant no.267802 "AnaMultiScale".

\section{References}

[1] W. Aschbacher, V. Jakšić, Y. Pautrat, and C.-A. Pillet. Transport properties of quasi-free fermions. J. Math. Phys., 48(3), P. 032101, 28 (2007).

[2] S. Attal, A. Joye, and C.-A. Pillet, editors. Open quantum systems. I, volume 1880 of Lecture Notes in Mathematics. Springer-Verlag, Berlin (2006).

[3] S. Attal, A. Joye, and C.-A. Pillet, editors. Open quantum systems. II, volume 1881 of Lecture Notes in Mathematics. Springer-Verlag, Berlin, 2006. The Markovian approach, Lecture notes from the Summer School held in Grenoble, June 16-July 4, 2003.

[4] S. Attal, A. Joye, and C.-A. Pillet, editors. Open quantum systems. III, volume 1882 of Lecture Notes in Mathematics. Springer-Verlag, Berlin, 2006. Recent developments, Lecture notes from the Summer School held in Grenoble, June 16-July 4, 2003.

[5] M. Baro, H.-Chr. Kaiser, H. Neidhardt, and J. Rehberg. A quantum transmitting Schrödinger-Poisson system. Rev. Math. Phys., 16(3), P. 281-330 (2004).

[6] H. Baumgärtel and M. Wollenberg. Mathematical scattering theory. Akademie-Verlag, Berlin (1983).

[7] M. Büttiker, Y. Imry, R. Landauer, and S. Pinhas. Generalized many-channel conductance formula with application to small rings. Phys. Rev. B, 31(10), P. 6207-6215 (May 1985).

[8] H. D. Cornean, P. Duclos, G. Nenciu, and R. Purice. Adiabatically switched-on electrical bias and the LandauerBüttiker formula. J. Math. Phys., 49(10), P. 102106, 20 (2008).

[9] H. D. Cornean, P. Duclos, and R. Purice. Adiabatic non-equilibrium steady states in the partition free approach. Ann. Henri Poincaré, 13(4), P. 827-856 (2012).

[10] H. D. Cornean, C. Gianesello, and V. A. Zagrebnov. A partition-free approach to transient and steady-state charge currents. J. Phys. A, 43(47), P. 474011, 15 (2010).

[11] H. D. Cornean, A. Jensen, and V. Moldoveanu. A rigorous proof of the Landauer-Büttiker formula. J. Math. Phys., 46(4), P. 042106, 28 (2005).

[12] H. D. Cornean, A. Jensen, and V. Moldoveanu. The Landauer-Büttiker formula and resonant quantum transport. Lecture Notes in Phys., 690, pages 45-53. Springer, Berlin (2006).

[13] H. D. Cornean, H. Neidhardt, L. Wilhelm, and V. A. Zagrebnov. The Cayley transform applied to non-interacting quantum transport. J. Funct. Anal., 266(3), P. 1421-1475 (2014).

[14] H. D. Cornean, H. Neidhardt, and V. A. Zagrebnov. The effect of time-dependent coupling on non-equilibrium steady states. Ann. Henri Poincaré, 10(1), P. 61-93 (2009).

[15] M. Damak. On the spectral theory of tensor product Hamiltonians. J. Operator Theory, 55(2), P. $253-268$ (2006).

[16] C. Gerry and P. Knight. Introductory Quantum Optics. Cambridge University Press, Cambridge (2005).

[17] T. Kato. Perturbation theory for linear operators. Classics in Mathematics. Springer-Verlag, Berlin (1995). Reprint of the 1980 edition.

[18] R. Landauer. Spatial Variation of Currents and Fields Due to Localized Scatterers in Metallic Conduction. IBM J. Res. Develop., 1(3), P. 223-231 (1957).

[19] H. Neidhardt, L. Wilhelm, and V. A. Zagrebnov. A new model for quantum dot light emitting-absorbing devices. J. Math. Phys., Anal., Geom., 10(3), P. 350-385 (2014).

[20] G. Nenciu. Independent electron model for open quantum systems: Landauer-Büttiker formula and strict positivity of the entropy production. J. Math. Phys., 48(3), P. 033302, 8 (2007). 